University of Louisville

ThinkIR: The University of Louisville's Institutional Repository

Electronic Theses and Dissertations

$5-2012$

\title{
Nanoscale diamond and carbon materials and architectures for field emission and thermionic energy conversion.
}

Santoshrupa Dumpala

University of Louisville

Follow this and additional works at: https://ir.library.louisville.edu/etd

\section{Recommended Citation}

Dumpala, Santoshrupa, "Nanoscale diamond and carbon materials and architectures for field emission and thermionic energy conversion." (2012). Electronic Theses and Dissertations. Paper 380.

https://doi.org/10.18297/etd/380

This Doctoral Dissertation is brought to you for free and open access by ThinkIR: The University of Louisville's Institutional Repository. It has been accepted for inclusion in Electronic Theses and Dissertations by an authorized administrator of ThinkIR: The University of Louisville's Institutional Repository. This title appears here courtesy of the author, who has retained all other copyrights. For more information, please contact thinkir@louisville.edu. 


\title{
NANOSCALE DIAMOND AND CARBON MATERIALS AND ARCHITECTURES FOR FIELD EMISSION AND THERMIONIC ENERGY CONVERSION
}

By

Santoshrupa Dumpala

B.Tech, Jawaharlal Nehru Technological University, India, 2005

\author{
A Dissertation \\ Submitted to the Faculty of the \\ Graduate School of the University of Louisville \\ In Partial Fulfillment of the Requirements \\ for the Degree of
}

Doctor of Philosophy

Department of Chemical Engineering

University of Louisville

Louisville, KY 40292

May 2012 
Copyright 2012 by Santoshrupa Dumpala

All rights are reserved 


\title{
NANOSCALE DIAMOND AND CARBON MATERIALS AND ARCHITECTURES FOR FIELD EMISSION AND THERMIONIC ENERGY CONVERSION
}

\author{
By \\ Santoshrupa Dumpala \\ B.Tech, Jawaharlal Nehru Technological University, India, 2005
}

A Dissertation Approved on

December 14, 2011

Bythe Following Dissertation Committee

Dr. Mahendra K. Sunkara (Dissertation Director)

Dr. Thomas L. J'Starr

Dr. Xiao-An Fu

Dr. Gamini U. Sumanasekera

Dr. Chakram \$. Javanthi

Dr. Richard P. Baldwin 


\section{DEDICATION}

This dissertation is dedicated to my parents

Meenavathi Dumpala and Ram Reddy Dumpala,

dear sister Nagamalleshwari

and all my dear friends. 


\section{ACKNOWLEDGEMENTS}

First and foremost I would like to thank my advisor, Prof. Mahendra K Sunkara for encouraging and providing me a great opportunity to be a part of his wonderful group with great independence to carry out some exciting work. I am indebted to my advisor for his constant guidance, motivation, encouragement and patience throughout my $\mathrm{PhD}$, a journey greatly cherished. My special thanks to Mr. Jeong H. Kim who is an expert in plasma devices for helping me with the experimental set ups and to Mr. Andriy Sherehiy for his great help with thermionic testing measurements.

I would like to thank Dr. Gamini Sumanasekera, Dr. Thomas Starr, Dr. Chakram Jayanthi, Dr. Xiao Fu and Dr. Richard Baldwin for serving on my dissertation committee. I appreciate their valuable feedback and suggestions.

I thank all the present and past members of our group for providing me with valuable suggestions, help and encouragement to make my stay here very enjoyable. I would also like to thank the Department of Chemical Engineering, University of Louisville for giving me this opportunity.

Lastly, I would like to thank my parents and my sister for their constant love, encouragement and blessings throughout my life.

Santoshrupa Dumpala

December 2012 


\section{ABSTRACT \\ NANOSCALE DIAMOND AND CARBON MATERIALS AND ARCHITECTURES \\ FOR FIELD EMISSION AND THERMIONIC ENERGY CONVERSION \\ Santoshrupa Dumpala \\ December $14^{\text {th }} 2011$}

More than $50 \%$ of the total energy produced is typically rejected in the form of waste heat from various processes. The grand challenge is that most of this waste heat is released at temperatures much lower than $1000 \mathrm{C}$, which makes it difficult to recover it using traditional methods which require higher operating temperatures for thermal energy conversion. In addition, these traditional methods involve different intermediate processes and do not offer direct conversion into electricity. In this regard, thermal energy conversion through thermionic emission can offer direct conversion of waste heat into electricity with highest theoretical efficiency. So, waste heat recovery through thermionic emission energy conversion is of great interest and is the motivation for the present work. However the greatest challenge involves the discovery or availability of the material with appropriate work functions and stability criteria.

To address the need for developing suitable materials toward thermionic energy conversion, we investigated phosphorus doping in individual diamond nanocrystals, conical carbon nanostructures (CCNTs) and diamond nanocrystals 
supported on conical carbon nanostructures. Hybrid architectures, diamond nanocrystals supported on high aspect ratio structures will allow the study of true performance of nanocrystals free from grain boundaries and also offer field enhancements.

First, the synthesis of CCNTs over large area and flat substrates is investigated. From the experimental results, we successfully synthesized CCNTs on planar graphite and tungsten foil substrates with areas as large as $\left(>0.5 \mathrm{~cm}^{2}\right)$. A detailed underlying nucleation and growth mechanism was also demonstrated supported with regrowth experiments and kinetic growth model. Secondly, selective nucleation of the diamond crystals on the tips or complete coating on CCNTs was demonstrated and a likely mechanism for the nucleation and growth of diamond crystals is also presented. Thirdly, the field and thermionic emission characteristics from the as synthesized CCNTs have shown to exhibit enhanced emission characteristics such as low turn-on voltages, large field enhancement factor and lower work function values owing to their higher aspect ratios and optimum density overcoming field screening effects. Finally, phosphorus doping into these individual diamond crystals and diamond films was performed and thermionic emission characteristics were studied. Work function values as low as $1.8 \mathrm{eV}$ from diamond films and $2.2 \mathrm{eV}$ from diamond crystals was obtained.

In summary, the main outcomes of this work include growth of large area CCNTs on flat substrates, discovery of the enhanced field and thermionic emission characteristics of CCNTs, selective nucleation and phosphorus doping of individual diamond nanocrystals on CCNTs free from grain boundaries and work function value as low as 2.2 $\mathrm{eV}$ from thermionic emission from these crystals. 


\section{TABLE OF CONTENTS}

PAGE

ACKNOWLEDGEMENTS...................................................... iv

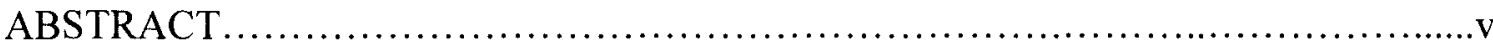

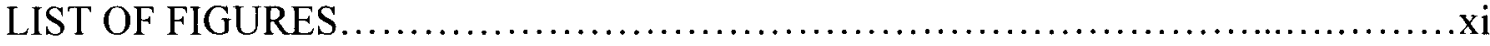

\section{CHAPTER}

1. Introduction

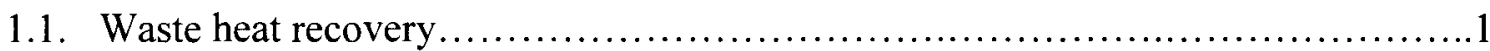

1.2. Thermionic emission concept and material challenges ..........................

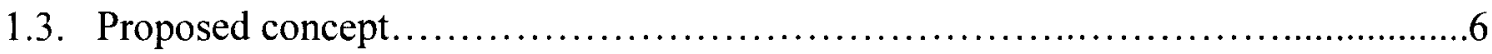

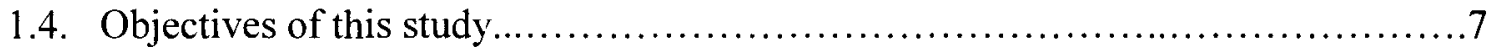

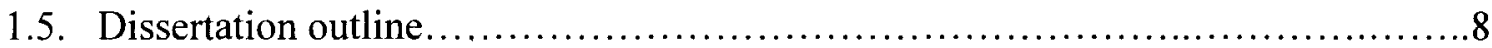

2. Background

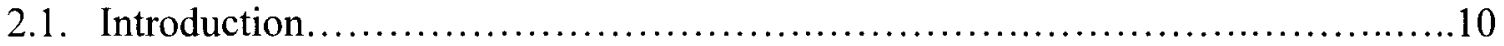

2.2. Thermionic emitter materials................................................. 10

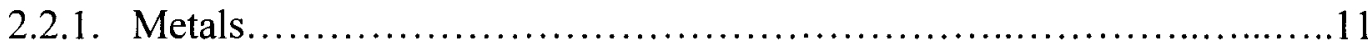

2.2.2. Carbon nanostructures.................................................. 15

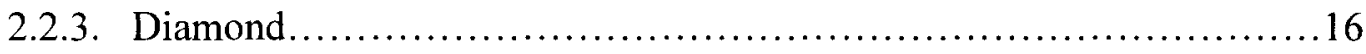

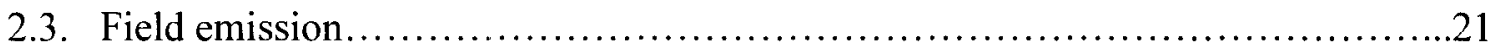

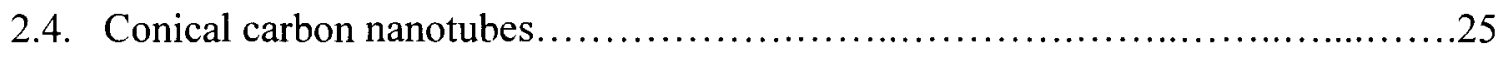

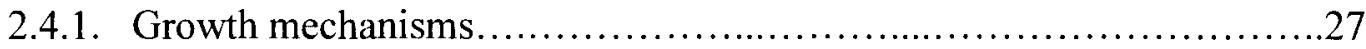


2.5. Diamond nucleation on carbon nanostructures................................... 31

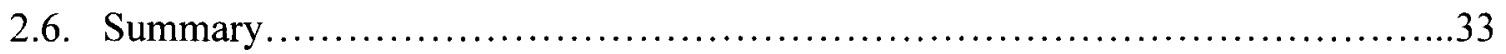

3. Experimental Techniques

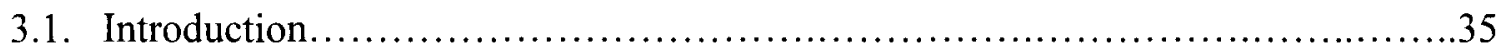

3.2. Nucleation and growth experiments..........................................

3.2.1. Growth of CCNTs using wire substrares...............................38

3.2.2. Growth of CCNTs using foil substrates...............................39

3.2.3. Diamond nucleation on CCNTs.................................40

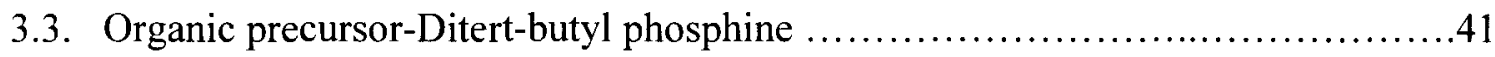

3.3.1. Determination of vapor pressure of Di-TBP ........................42

3.3.2. P-doping into diamond crystals on CCNTs ...........................43

3.3.3. P-doping into diamond films......................................44

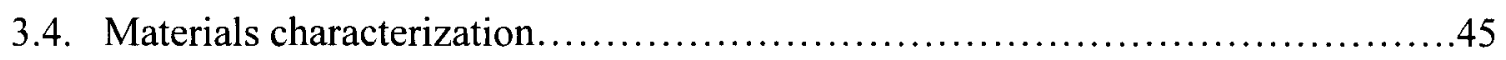

3.4.1. Ultraviolet photoelectron spectroscopy ................................45

3.4.2. Field emission characterization.....................................47

3.4.3. Thermionic emission characterization ..............................48

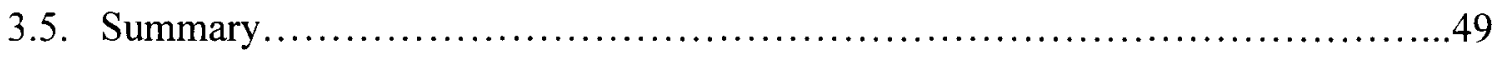

4. Large area synthesis of CCNTs on planar substrates

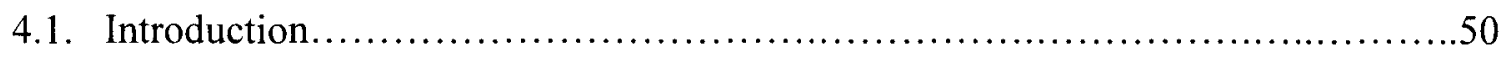

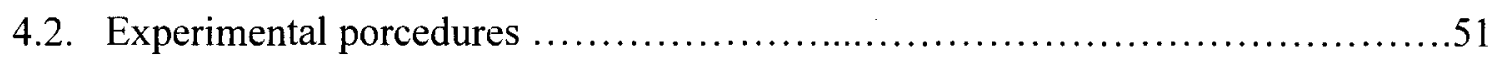

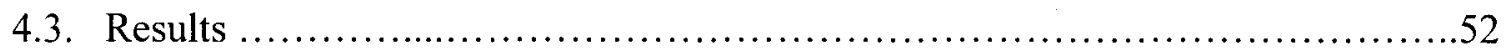

4.3.1. CCNTs with platinum catalyst....................................52

4.3.2. CCNTs with iron and nickel catalyst..................................54 
4.3.3. Role of the catalyst and graphite rod...............................56

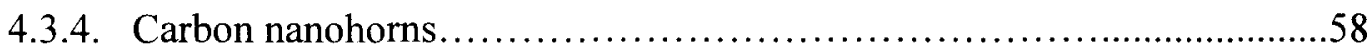

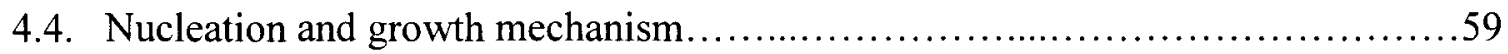

4.4.1. Regrowth experiments............................................63

4.4.2. Kinetic growth model............................................65

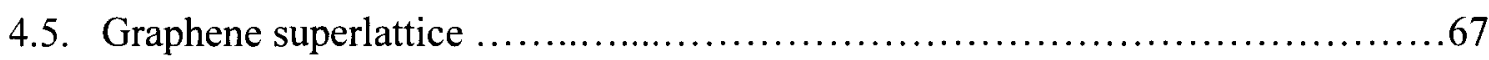

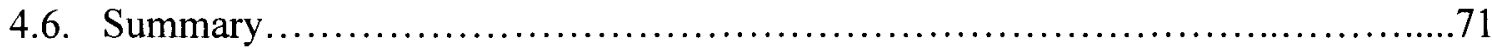

5. Field and thermionic emission characteristics of CCNT arrays

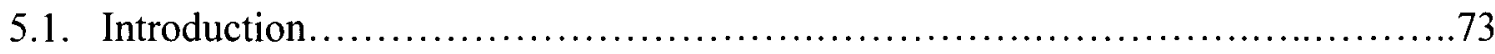

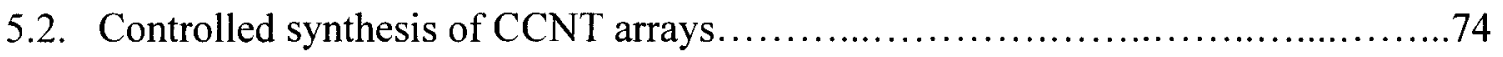

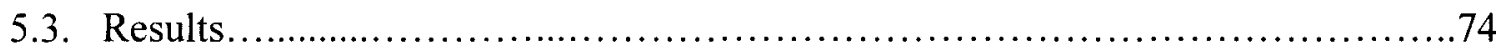

5.3.1. CCNT arrays with varying aspect ratio and density ....................74

5.3.2. Field emission characteristics....................................... 78

5.3.3. Thermionic emission characteristics................................ 82

5.3.4. Ultraviolet photo spectroscopy studies ...............................86

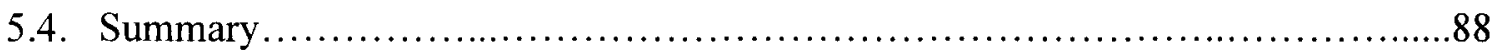

6. Diamond tipped and coated CCNT arraysanodes

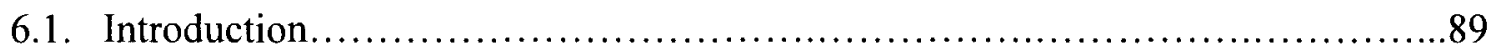

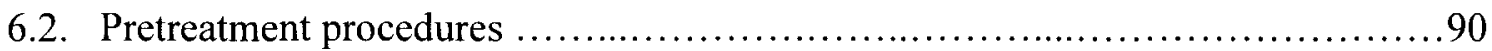

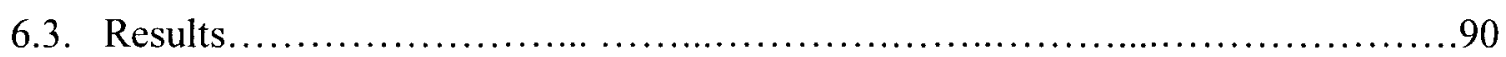

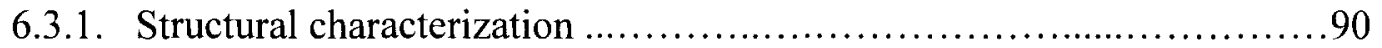

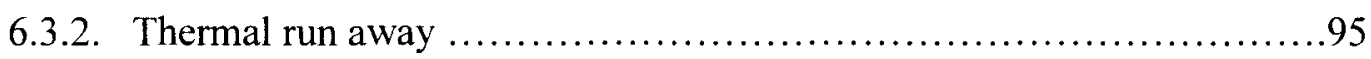

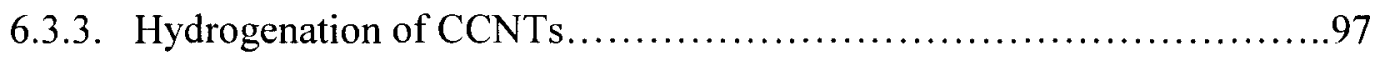


6.3.4. Carbon onions................................................... 100

6.4. Nucleation and growth mechanism..................................... 102

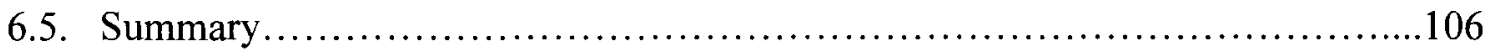

7. Thermionic emission from phosphorus doped diamond crystals

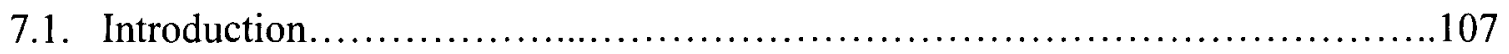

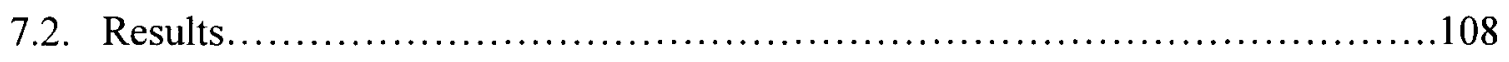

7.2.1. Thermionic emission from diamond crystals on CCNTs.................108

7.2.2. UPS studies on undoped and P-doped diamond films................113

7.2.3. Phosphorus doped diamond microcrystals..........................118

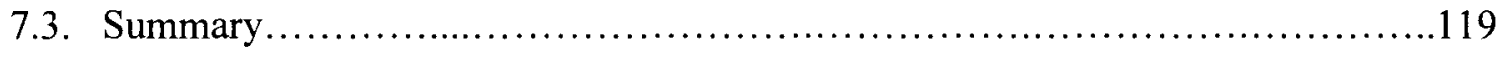

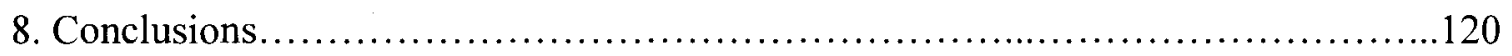

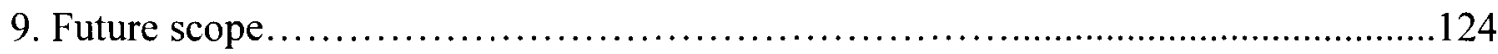

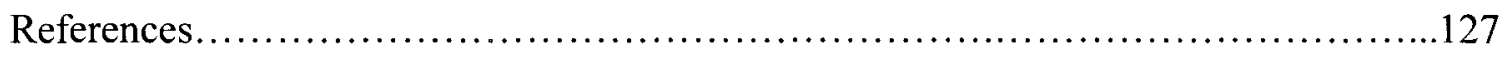

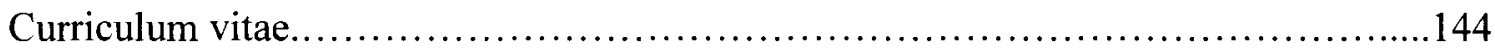




\section{LIST OF FIGURES}

FIGURE

PAGE

1.1. Schematic representation of band energy diagram for thermionic emission process

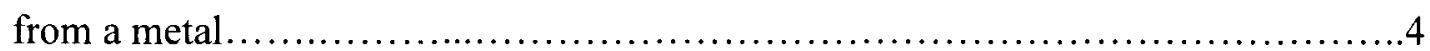

1.2. Schematic illustration of diamond nanocrystals at the tips of conical carbon nanotubes 6

2.1. Schematic representation band energy diagram for electron emission from a metal with and without the application of external electric field .22

2.2. Schematic of (a) nucleation and growth of initial nanotube before etching of the catalyst (b) formation of conical morphology resulting from shrinking of the catalyst

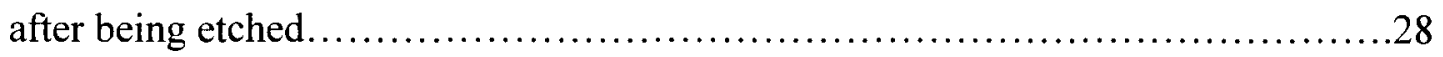

2.3. Schematic illustration of a catalyst-assisted basal growth of a central carbon nanotube followed by continuous nucleation of new graphene walls for thickening the base while tapering vertically in thickness forming a conical structure

2.4. Schematic illustration of catalyst assisted tip-led growth of a central carbon nanotube followed by the evaporation of the catalyst particle and simultaneous deposition and etching of the helical graphite edge planes leading to the conical morphology ........30 
2.5. Schematic representation of (a) tip led growth of vertically aligned carbon nanotube (b) evolution of conical morphology due to additional precipitation of carbon on the outer wall of the inner nanotube 31

3.1. Schematic of MW CVD reactor............................................... 37

3.2. Schematic illustration of growth of CCNTs on platinum wire substrates.............39

3.3. Schematic illustration of the series of steps for the synthesis of CCNTs on foil substrates. . .40

3.4. Schematic representation of horizontal positioning of the platinum wire coated with CCNT array for diamond nucleation.

3.5. Schematic illustration of bubbler set up for phosphorus doping 42

3.6. Schematic illustration of UPS chamber. 47

3.7. Field emission experimental set up showing (a) picture of the experimental chamber (b) schematic illustration of experimental set up inside the chamber. .48

3.8. Schematic illustrations of thermionic emission experimental set-ups for (a) for CCNT's on platinum wire and (b) for CCNT's (or CCNT's with diamond) on graphite foil

4.1. Schematic illustration of an experimental configuration for CCNTs with in-situ nickel wire

4.2. SEM images showing (a) an array of CCNTs on graphite foil, (b) an individual CCNT with inset showing the open-ended tip and (c) surface of CCNT consisting of concentric cylindrical graphene sheets and (d) non epitaxial coiling of graphene sheets around the CCNT 
4.3.SEM images of (a) an array of CCNTs with different morphologies resulted during experiments with nickel wire, (b) an individual CCNT with a blunt tip and (c) an individual CCNT with inverted conical tip .54

4.4. TEM images of CCNTs showing the absence of catalyst (a) CCNTs with sharp tip, (b) CCNTs with blunt tip, (c) CCNTs with inset showing the presence of a hollow core, (d) CCNTs with inverted conical tip with inset showing the high resolution image of the tip consisting of graphitic planes and (e) high-resolution image of a kink at the tip with inset showing the abrupt change in the hollow core................56

4.5. SEM images of (a) array of CCNTs (b) close up image of the individual CCNT with

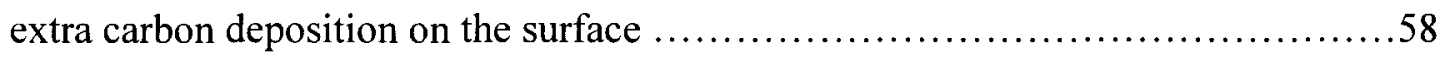

4.6. TEM images of (a) bundle of nanohorns and (b) a high-resolution image of individual multi-walled nanohorn .59

4.7. TEM image of CCNT with its elemental analysis revealing the absence of any metal catalyst at either tip or base.

4.8. SEM image showing the (a) side, cross-sectional view of a CCNT grown on tungsten foil substrate and (b) SEM image of a broken CCNT with inset showing the absence of a catalyst particle at the base.

4.9. A schematic illustration of the suggested nucleation and growth mechanism for CCNTs (a) formation of small catalyst clusters on the substrate, (b) nucleation of a MWNT, (c) continued catalyst-assisted growth of MWNT along with the nucleation of new graphene layers, (d) evaporation of the catalyst with continued growth of epitaxial layers and (e) continued radial growth via addition of graphene layers and steady state vertical growth via addition of carbon atoms...... 
4.10. SEM image of (a) CCNTs etched to straight MWNTs and (b) regrowth of etched CCNT to conical structures with inset showing magnified view of one of the CCNTs circled. .64

4.11. (a) Plot showing the length of conical structures at various time scales during the growth and (b) plot showing linear relationship between the growth rate and the ratio of the diameter to length $(d / l)$ of the conical structures.

4.12. (a) TEM micrograph ( $250 \mathrm{~nm} 350 \mathrm{~nm})$ of FLG graphene prepared by CVD. A superlattice is seen in the top section and parallel bands are seen in the bottom part (highlighted outlines), (b) FFT of HRTEM image indexed using [0001] graphite zone axis. Notice the radial splitting of $1.3^{\circ}$ present for all (10-10)-type spots. An enlarged view of the $(0-110)$ spot splitting is shown in the inset. .69

4.13. Model of two layer graphene sheets (blue bottom layer and red top layer). The top part of the top (red) layer is rotated through $5^{\circ}$ and the shape of the bottom part is changed to a parallelogram with an $85^{\circ}$ top right hand corner. The two sections are continuous without any break in the chemical bonds. Regions of aa, aba, and $\sim$ aa are indicated below the corresponding regions. Schematics of each layer are shown for clarity 71

5.1. SEM images of array of CCNTs of (a) sample 1 (b) sample 2 and (c) sample 3, with insets showing the enlarged view of the corresponding CCNT tip $\ldots \ldots \ldots \ldots \ldots \ldots . . . .75$

5.2. SEM images of the individual CCNT of (a) sample 1 (b) sample 2 and (c) sample 3 , illustrating the variation in the wall structure and aspect ratio .77

5.3. SEM images of CCNT arrays of (a) sample 4 (b) sample 5, with insets showing the enlarged view of the corresponding CCNT tip .77 
5.4. Field emission properties from the CCNTs showing the plots of (a) Current vs. the macroscopic electric field for various inter-electrode distances (d) for sample 1, (b) corresponding F-N plots............................................ 79

5.5. Plots of (a) field enhancement factor $\beta$ and (b) turn-on electric field as a function of

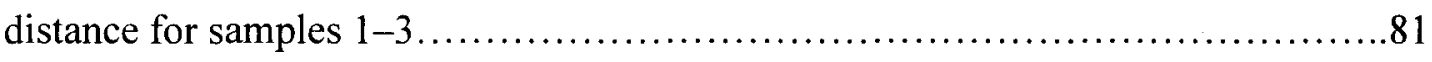

5.6. Field emission properties from the CCNTs showing the plots of (a) Current Vs. the macroscopic electric field for sample 4 and sample 5, (b) corresponding F-N plots...81

5.7. FETE plots of $I-V$ characteristics from CCNT arrays at various temperatures (a) In linear scale, with enlarged view in inset and (b) Ln-linear scale..................84

5.8. TE characteristics of CCNTs (a) $\operatorname{Ln}(I)$ vs $V^{1 / 2}$, (b) Richardson plot of $I$ Vs T and (c) linear fitting of $\operatorname{Ln}\left(\frac{I_{0}}{T^{2}}\right)_{\mathrm{vs}} \frac{1}{T}$ (straight line) to the experimental data (solid circles)85

5.9. TE characteristics of CCNTs (a) $\operatorname{Ln}(I)$ vs $V^{\mathrm{l} / 2}$, (b) Richardson plot of $I$ Vs T and (c) linear fitting of $\operatorname{Ln}\left(\frac{I_{0}}{T^{2}}\right)_{\text {vs }} \frac{1}{T}$ (straight line) to the experimental data (solid circles)86

5.10. Low kinetic-energy He - I (21.23 eV) spectra of CCNT arrays on (a) platinum wire and (b) graphite foil substrate with insets showing low kinetic cut-off energy

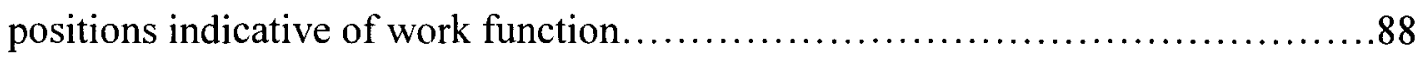

6.1. SEM image of (a) an array of as synthesized CCNTs on platinum wire substrate, (b) individual CCNT exhibiting conical geometry ............................. 91

6.2. SEM images of (a) selective growth of sub-micron scale diamond crystals on tips of CCNTs grown for $20 \mathrm{~min}$, (b) micron scale diamond crystals with growth duration of about $3 \mathrm{hr}$, (c) an individual CCNT etched to CNT with rough faceting diamond 
crystal at the tip and (d) Visible raman spectrum showing the diamond characteristic peak at $1332 \mathrm{~cm}^{-1}$ .92

6.3. SEM images showing diamond nucleation on CCNT as a result of pretreatment involving sonic dispersion bath of (a) micron-scale diamond powder, (b) submicronscale diamond powder, pretreatment involving electrophoretic seeding in dispersions of (c) micron-scale, (d) sub-micron scale diamond powder and (e) UV raman spectrum confirming the presence of diamond exhibiting diamond characteristic peak at $1332 \mathrm{~cm}^{-1}$

6.4. (a) Bright field TEM image of the individual diamond crystal at the tip of etched CCNT, (b) a schematic illustration of thermal run away scenario of the diamond crystal at the tip of the nanotube and (c) plot showing the estimated temperature rise during diamond growth as a function of size of the diamond crystal .97

6.5. SEM images of an array of (a) as synthesized CCNTs with tapered morphology with various lengths and (b) CCNTs etched to CNTs after hydrogen plasma etching.....98

6.6. SEM image of an individual as synthesized CCNT (a) before etching, (b) after etching with insets showing the HRTEM image of the respective wall structures and (c) bright field image of a partially etched CCNT showing the partial etching of the wall structure with inset showing the HRTEM image of the closing of the tip......100

6.7. (a) SEM image showing several bright clusters on the sidewall of CCNT, (b) HRTEM image of the one of the bight clusters exhibiting the carbon onion structure and (c) a schematic illustration of mechanism of the curling of CCNT walls at the damage sites created during the pretreatment step 
6.8. (a) HTREM image of a diamond crystal nucleated within the subsurface layer, (b) A subsurface diamond crystal is shown with regions labeled as 'A' for tube walls, ' $\mathrm{B}$ ' for the region between diamond and tube wall, and ' $\mathrm{C}$ ' for the inner diamond crystal. The inset is an FFT pattern showing the diffraction bands and maxima resulting from both multi-walled carbon tube and diamond, respectively, (c) FFT reconstructed image from the maxima corresponding to diamond from region ' $\mathrm{C}$ ' and (d) FFT reconstructed image from the diffraction corresponding to the multiwall graphite from region ' $\mathrm{A}$ '. (e) ELNES of carbon-K edge showing a mixture of diamond and multiwalled carbon tube.

6.9. SEM images showing CNPs treated with boron carbide powder (a) Shows the complete coating of diamond crystals along the length of CNP clearly depicting the spiral growth of diamond crystals and (b) shows large array of CNPs coated with diamond crystals

7.1. SEM images of individual (a) as synthesized CCNT, (b) undoped diamond coated CCNT and (c) P-doped diamond coated CCNT on graphite foil substrates. 109

7.2. Secondary ion mass spectroscopy (SIMS) depth profile of phosphorus in P-doped diamond crystals on CCNTs

7.3. Thermionic emission measurements plots of $\mathrm{P}$ doped diamond coated CCNTs (a) showing thermionic $\mathrm{I}-\mathrm{V}$ characteristics, (b) showing $\mathrm{Ln}(\mathrm{I})$ vs $\mathrm{U}^{1 / 2}$ curves for various temperatures in ${ }^{\circ} \mathrm{C}$

7.4. Thermionic emission data (dots) and data-fit (line) to Richardso-Dushman equation from as synthesized CCNTs, CCNT coated with undoped diamond and CCNT coated with phosphorus doped diamond 
7.5. UPS spectra of glod film showing Fermi level positions at (a) $21.23 \mathrm{eV}$ for He- I (b) $40.83 \mathrm{eV}$ for He-II radiations 114

7.6. Low kinetic-energy $\mathrm{He}-\mathrm{I}(21.23 \mathrm{eV})$ spectra of a) undoped diamond films b) phosphorus doped diamond film showing low kinetic cut-off energy positions indicative of work function and a high intensity cut-off position of conduction band

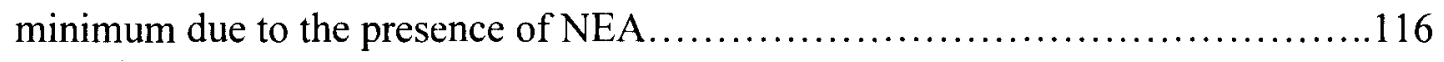

7.7. Secondary ion mass spectroscopy (SIMS) depth profile of phosphorus in P-doped diamond films on silicon wafer............................................ 117

9.1. Schematic illustration of atom probe tomography experimental set up.............125 


\section{CHAPTER 1}

\section{INTRODUCTION}

Energy consumption is tremendously increasing every year in the United States. This is compounded by the fact that a significant fraction of energy being produced is basically rejected (54.64\%) according to U.S. Department of Energy reports ${ }^{1}$. Typically, this rejected energy is in the form of waste heat which mainly arises from both equipment inefficiencies and from thermodynamic limitations of equipment and processes. Some of the sources of waste heat include thermal power plants, vehicle engines, and industrial waste heat from oil refineries and steel plants. Though some of the waste heat losses from industrial processes are inevitable, particular facilities can reduce these losses either by improving equipment efficiency or installing waste heat recovery technologies.

\subsection{Waste heat recovery}

Waste heat recovery involves capturing and reusing the waste heat in industrial processes either for heating or for generating mechanical or electrical work. Thus, heat recovery technologies frequently reduce the operating costs by increasing their energy productivity. Different methods for waste heat recovery include transferring heat between gases and/or liquids, or to the load entering furnaces, generating mechanical and/or electrical power, or using waste heat with a heat pump for heating or cooling. 
Among these different methods, conversion of waste heat to electrical power is of most interest in the present context. While traditional power cycles involve use of heat for creating mechanical energy and ultimately electrical energy, new technologies are being developed that can generate electricity directly from heat. These include thermoelectric, thermionic, and piezoelectric devices. An important factor considered for waste heat recovery is the thermodynamic limitation on power generation at different temperatures. The efficiency of power generation is highly dependent on the temperature of the waste heat source. Though power generation from waste heat has been limited to only medium to high temperature waste heat sources, advances in alternate power cycles can increase the feasibility of generation at low temperatures. Thermionic emission offers several advantages compared to its counterparts (such as thermoelectric) including; 1. Thermionics relies upon ballistic emission of electrons which have faster time of flight from the emitter to the opposite electrode. On the other hand, thermoelectrics relies upon diffusive electronic transfer with low mobilities which lead to lower current densities compared to thermionics. 2. Thermionics does not require complicated structures - i.e., it can be totally established on the thermal gradient and the work function of the collector relative to the emitter; thus, engineering and execution of the devices can be greatly simplified $^{2}$. In addition, some other attractive features of thermionic power generation include low maintenance, scalability, high reliability, high power density, and high efficiencies at high temperatures ${ }^{3}$. However, higher efficiencies can also be achieved at lower temperatures with the development of new materials with suitable work functions. This is the motivation for the present work. 


\subsection{Thermionic emission concept and materials challenges}

The thermionic emission process is used as a source of electrons in many applications including scanning electron microscopes, cathode ray tubes, X-ray tubes, mass spectrometers, vacuum gauges, fluorescent lighting, and other scientific instruments. These thermionic converters can also be operated in combination with several other heat sources, such as nuclear power plants, solar radiation and fossil fuels ${ }^{4}$. Thermionic emission was first discovered by Edison in 1883, and is known as Edison effect i.e. emission of electrons from the hot surface into vacuum in a vacuum tube. In 1899, it was demonstrated by Thomson ${ }^{5}$ that thermionic emission consisted of electrons. In early 1910 , there was a controversy in the proposed theories for thermionic emission. One theory suggested that it was a fundamental property of a cathode material and the escape of electrons was due to purely thermal increase of their kinetic energy. Another suggested that the emission of the electrons was a result of chemical reactions between the cathode and residual gases. However, Richardson demonstrated that it was purely the result of thermal effects and thermionic emission was first described by Richardson's equation ${ }^{6}$ expressed as

$$
J=A T^{2} e^{-\left(\frac{\Phi}{k T}\right)}
$$

Where $\mathrm{J}$, is the emission current density $\left(\mathrm{A} / \mathrm{cm}^{2}\right), \mathrm{T}$ is the temperature $(\mathrm{K}), \mathrm{A}$ is the Richardson constant $\left(\mathrm{A} / \mathrm{cm}^{2} \mathrm{~K}^{2}\right), \mathrm{k}$ is the Boltzmann constant, and $\phi$ is the work function $(\mathrm{eV})$. Figure 1.1 shows the schematic illustration of a conventional vacuum thermionic emission process operated on the basis of ejection of high-energy electrons overcoming the potential barrier (i.e., work function $\phi$ ) from a hot surface and their collection at the 
cooler surface separated by a vacuum gap. This process can provide electrical energy without the inclusion of any moving parts, thus making it a highly efficient and low maintenance power source.

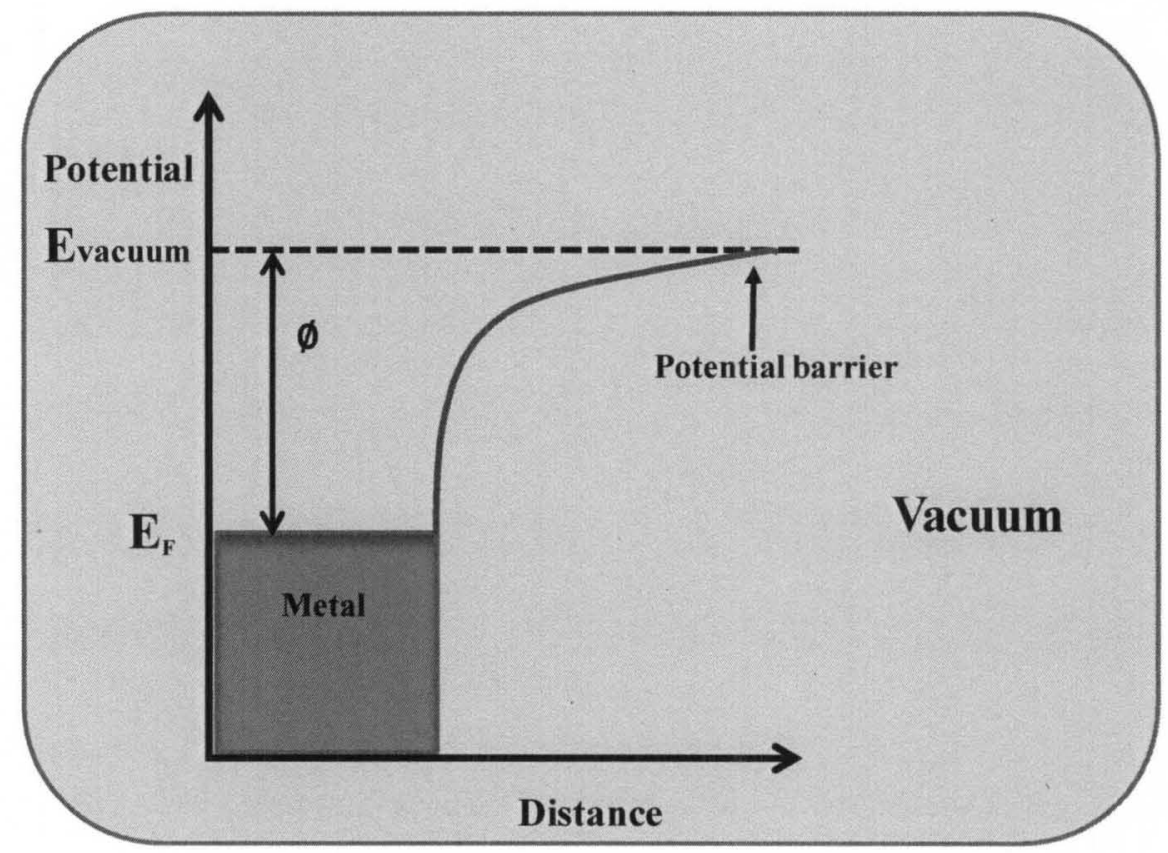

Figure 1.1 Schematic representation of band energy diagram for thermionic emission process from a metal

From the Richardson's equation, it is evident that the emission current mainly depends on the temperature and the work function of the emitter material. Early thermionic energy converters were based on electron emission from flat metal surfaces. However, as the work functions of most of metals were in the range of $4.5-5.1 \mathrm{eV}$, higher temperatures up to $2500{ }^{\circ} \mathrm{K}^{7}$ are required for large thermionic currents. Though work function values were lowered by some special electropositive surface coatings on these 
metals, they suffered from either the loss of coatings, leading to shorter life times or higher operating temperature $\left(>1000^{\circ} \mathrm{K}\right)^{7}$.

Thus a cathode material with higher thermal and electrical stability and lower work function is necessary for thermionic emission at temperature less than $1000{ }^{\circ} \mathrm{K}$ for efficient waste heat recovery. Among various carbon based materials, diamond with its exceptional physical and electronic properties is of great interest thermionic emission energy conversion applications. Unique features of diamond include high carrier mobilities, highest thermal conductivity at room temperature, high saturation velocity, high breakdown electric field, the highest elastic modulus, highest mechanical hardness, low coefficient of thermal expansion, wide band-gap, low coefficient of friction, and high resistance to harsh environments including radiation, chemicals, and corrosion ${ }^{8.9}$. With these excellent properties, diamond is expected to be a promising candidate for the applications such as high-power and high-frequency devices ${ }^{10}$, electron emission ${ }^{11}$, and light emitting diodes ${ }^{12}$. Moreover, diamond grown using the chemical vapor deposition method exhibits a special characteristic feature called negative electron affinity (NEA) i.e., the presence of a vacuum level below the conduction band minimum $(\chi=-1.27 \mathrm{eV})$ due to hydrogen termination on the surface ${ }^{13}$. Due to NEA, the electrons that are present in the conduction band can readily escape to the surface. In addition, n-type doping of diamond has been shown to introduce shallow donor levels close to the conduction band $^{14}$, which in turn can lower the work function values by raising the Fermi level compared to p-type doping. Together with NEA and highest thermal conductivity, diamond finds great potential in thermionic energy conversion of waste heat directly into electricity. 
Although several methods were proposed for lowering the work function values either by surface treatments ${ }^{15,16}$ or n-type doping into diamond using several dopants ${ }^{14}$, there exists only few reposts on the study of thermionic emission properties from n-type diamond films. Moreover, n-type doping into diamond especially in the case of phosphorus which has shown to introduce shallow donor level $(0.6 \mathrm{eV})$ below the conduction band has been very challenging due to its large atomic radii compared to carbon and its incorporation into diamond lattice has been very difficult ${ }^{17}$. In addition, all the above reports involved the study of diamond films, which prevented the study of a true diamond crystal free from grain boundaries. Currently there exists no technique to synthesize and study the thermionic emission from individual phosphorus doped diamond nanocrystals.

\subsection{Proposed concept}

To address these challenges, we propose to study phosphorus doping in individual diamond nanocrystals supported on tips of conical carbon nanotube arrays (CCNTs). Such hybrid architecture as shown

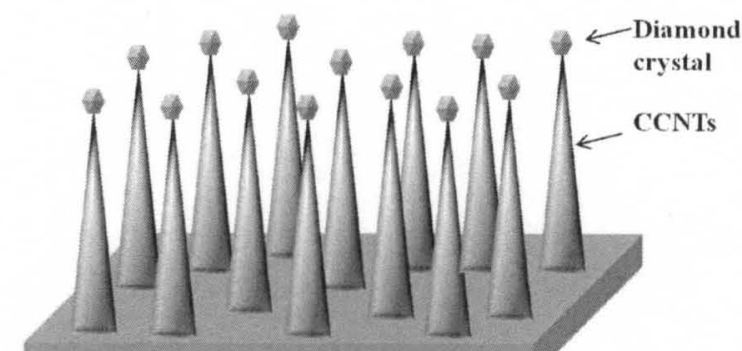
in Figure 1.2, is essential and unique because (a) true performance of nanocrystals can be possible without interference from Figure 1.2 Schematic illustration of diamond nanocrystals at the tips of conical carbon nanotubes.

grain boundaries and (b) the incorporation of dopants in to nanoscale crystals can be 
enhanced due to high surface to volume ratio. In the case of n-type doping with $\mathrm{P}$ or $\mathrm{S}$, high concentrations of dopants were reported near the surface of the crystal compared to the bulk ${ }^{18}$. These dopants have been shown to exhibit the tendency to segregate to the surface, which can be attributed to the presence of large number of vacancies at the surface. Thus, in the case of nanocrystals with increased surface area, the segregation of the dopants can be expected to increase the overall conductivity as doping into the bulk of a nanocrystal is the same as doping the surface. In addition, this architecture proposed here, i.e., diamond nanocrystals at tips of CCNT arrays, may exhibit enhanced field and thermionic emission properties combining the advantages of both the structures.

\subsection{Objectives of this study}

Towards achieving the proposed concept, this dissertation is focused on understanding both the nucleation and growth mechanism of individual diamond crystals on conical carbon structures and their phosphorus doping for determination of thermionic emission characteristics. Specific goals include:

a) Synthesis of CCNT arrays over a large area on flat substrates and investigate the underlining nucleation and growth mechanisms of CCNTs for achieving control over their density and aspect ratio. These mechanically robust and electrically conducting CCNT structures can potentially provide stable electrical contact to the diamond crystals for enhanced thermionic/field emission from the structures.

b) Synthesis and investigation of nucleation and growth mechanism of diamond crystals on CCNT arrays for achieving the hybrid architecture with proper control over the size and density of the diamond crystals. 
c) Examine the field and thermionic emission characteristics of as synthesized CCNTs for understanding of the field and thermionic emission characteristics of the hybrid architecture.

d) Achieve phosphorus doping and studying the thermionic emission characteristics from these individual diamond crystals.

\subsection{Dissertation outline}

This dissertation is divided into 9 chapters. Chapter 2 gives an overview of the extensive literature on the different thermionic materials employed to date and the challenges encountered. A review of different conical carbon structures is also presented along with several challenges in understanding the nucleation of conical carbon structure, individual diamond crystals, and phosphorus doping in diamond for obtaining stable and low work function material for thermionic emission.

Chapter 3 provides details of all the experimental techniques employed for nucleation and growth of carbon and diamond nano structures and characterization tools used to analyze their structure and morphology and to study their field and thermionic emission properties.

Chapter 4 elucidates the nucleation and growth mechanism of CCNTs synthesized over a large area on flat substrates by chemical vapor deposition (CVD). This chapter describes different experimental finding and a kinetic growth model for understanding the evolution of conical morphology. 
Chapter 5 presents the synthesis of CCNTs with varying densities and aspect ratios and their field emission and thermionic emission characteristics of as synthesized CCNT arrays.

Chapter 6 describes the growth of individual diamond crystals on CCNT array substrates. Also described are different pretreatment techniques for varying the size and the density of the diamond crystals on CCNTs and the underlining nucleation mechanism.

Chapter 7 describes the growth and thermionic emission characteristics/work function values of phosphorus doped individual diamond crystals and diamond films.

Chapters 8 and 9 provide conclusions and the results of hybrid architecture and phosphorus doping and future scope. 


\section{CHAPTER 2 \\ BACKGROUND}

\subsection{Introduction}

In this chapter, an overview of different thermionic cathode materials and various methods employed to date is described along with the challenges encountered in obtaining a stable and low work function thermionic emitter. Field emission characteristics from various carbon nanostructures are also presented. An overview of the current status and challenges of n-type doping (especially phosphorus) doping into diamond is presented. Nucleation and growth mechanisms of different conical carbon nanostructures (CCNTs) and diamond on carbon nanostrucutres is also reviewed.

\subsection{Thermionic emitter materials}

As mentioned earlier, small work function materials are highly desired for efficient thermionic emission at lower temperatures $\left(<1000^{\circ} \mathrm{K}\right)$. Work function $(\phi)$ is the minimum amount of energy required to remove an electron from the material to the point immediately outside the surface, or it can also be described as the minimum energy required for moving an electron from Fermi level to vacuum. Other parameters pertinent to the choice of the cathode material include the material's thermal and electrical stability and its compatibility with the operating environment. The maximum current density that can be extracted from the emitter and its life time also limits the choice of the material. 
Although an extensive search for materials and different treatment and processing methods has been carried out with limited progress, there is still a great need for the appropriate material satisfying all the conditions.

In general, hot emitters are classified into two categories; directly and indirectly heated cathodes, based on the method of heat generation. Directly heated cathodes consist of a metallic filament, usually of tungsten, which emits electrons when a DC current is passed through it. On the other hand, indirectly heated cathodes consist of an emitter heated to a desired temperature value for the emission on an electrically insulated but thermally conducting heater for heating the emitter. These cathodes allow use of large area of the emitter material for achieving high discharge current compared to direct heated cathodes which are limited to use of only the filament. In addition, indirectly heated cathodes guarantee much longer lifetimes compared to their counterparts ${ }^{19}$. Thus, the indirect heating technique is of most interest in this context. A review of several materials with different surface coatings employed so far and their limitations and existing challenges have been discussed below.

\subsubsection{Metals}

Early thermionic energy converters were mostly based on electron emission from flat metal surfaces. However, since the work functions of the most of the metals are in the range of 4.6-5.1eV, large thermionic currents required higher temperatures, up to 2500 ${ }^{\circ} \mathrm{K}^{7}$, which limit the operational lifetime of the converter. In addition, increasing the temperature does not necessarily ensure observable electron emission due to possible evaporation, melting, and decomposition of materials during the heating. Thus, only 
refractory materials like tungsten, tantalum and rhenium are useful as thermionic sources of electrons due to their high melting points ${ }^{7}$. However, if the thermionic converter can be made to operate efficiently at lower temperatures by lowering the emission barrier (work function), their potential applications can be greatly increased along with life time.

(a) Surface coatings: One of the methods developed for lowering the potential barrier is use of some special surface coatings on these metals.

Electropositive Material: Deposition of a monolayer of an electropositive material onto metals has been shown to lower the work function values appreciably. In the case of thoriated tungsten, $\phi$ was lowered to $2.7 \mathrm{eV}^{7}$. However, high operating temperature of the cathode of $2000^{\circ} \mathrm{K}$ limited its usage. On the other hand, a monolayer of barium on tungsten lowered $\phi$ about $2 \mathrm{eV}$ and operated at $1200-1400{ }^{\circ} \mathrm{K}$. But these cathodes suffered from poisoning by oxidizing gases ${ }^{7}$. Also, formation of the monolayer involves several time consuming and complicated steps. A monolayer of cesium on tungsten $^{7}$ and molybdenum ${ }^{20}$ has shown to lower $\phi$ to $1.4-1.6 \mathrm{eV}$. Due to very high volatility of the cesium, the monolayer can only be stably operated in cesium vapor, thus requiring a continuous replenishment strategy ${ }^{7}$.

Oxides: An oxide coating consisting of barium strontium oxide mixed with calcium oxide on nickel has shown to reduce $\phi$ to around $1.5 \mathrm{eV}$ with operating temperature of $1000^{\circ} \mathrm{K}$. However, they also suffered from either the loss of coatings through ion sputtering leading to shorter life times or sensitive to poisoning resulting in irrecoverable changes in the coating ${ }^{7}$. Other refractory oxide coatings include coating of thoria (thorium oxide) on tungsten giving a value of $\phi$ about $3.1 \mathrm{eV}$ with very high 
operating temperatures of $2000{ }^{\circ} \mathrm{K}^{7}$. Some of the rare earth oxide coatings such as gadolinia and yttria ${ }^{21}$ on tungsten resulted in $\phi$ of about $2.8 \mathrm{eV}$ at $1500{ }^{\circ} \mathrm{K}$. But their practical applications have been very limited ${ }^{7}$.

Refractory compounds: Among the other compounds studied as thermionic cathodes exhibiting low work function values include borides, nitrides and carbides. The most important borides known as excellent thermionic emitter is lanthanum hexaboride $\left(\mathrm{LaB}_{6}\right)$ exhibiting work function value of $2.4 \mathrm{eV}$ at $1680{ }^{\circ} \mathrm{C}^{22}$. Since indirectly heated $\mathrm{LaB}_{6}$ consume considerable amount of power, $\mathrm{LaB}_{6}$ coated tantalum ${ }^{23}$ and tungsten ${ }^{24}$ cathodes have shown to exhibit advantages of ease of fabrication and low power consumption. However, borides tend to suffer from the migration of boron into any carrier metal at excessive rate at high temperatures. Other compounds of practical interest include uranium and zirconium ${ }^{7}$ carbides, but they required higher temperatures around $2000^{\circ} \mathrm{K}$ owing to work function values of $3.1-3.2 \mathrm{eV}$.

From all the above reports, it is evident that though work function values were lowered using different coatings, stable emission is limited at temperatures $<1000^{\circ} \mathrm{K}$

(b) Space charge: While achieving low work function value is essential for efficient thermionic emission, another major limitation in the use of flat metal surfaces is the space charge effect. If the anode voltage is small, electrons travel slowly in vacuum resulting in formation of a continuum of charge distributed over a region termed as "space charge". This effect generally exists in dielectric medium as in the case of conducting medium, this charge tends to be neutralized or screened. This effect induces an electrostatic potential that is negative in front of the cathode and, therefore presents a barrier. In other 
words, this charge-build up screens other electrons from being efficiently released into vacuum which may in turn return to the cathode.

There are few approaches which can be considered for mitigation of this space charge. Employing a narrow cathode-anode spacing $(<5 \mu \mathrm{m})$ configurations can result in overcoming this space charge. However, the manufacturing of such systems becomes difficult. Incorporation of positive ion species such as cesium vapors neutralizing this negative space charge have shown to be successfully used in thermionic energy conversion applications. However, these processes resulted in bulky design requiring temperatures greater than $1000{ }^{\circ} \mathrm{C}$, thus not being suitable for terrestrial and mobile applications $^{25}$. A new concept of incorporating a molecular assisted charge transport between the cathode and anode surfaces has been demonstrated towards the mitigation of space charge ${ }^{26}$. Another method for reducing the space charge is by applying external electric field to accelerate the electrons towards the anode. This also results in bending of the potential barrier called as Schottky effect ${ }^{7}$. Thus field enhanced thermionic emission (FETE) gained much attention due to lower power consumption compared to TE. However, if the applied electric field is large enough, it would lead to field emission described later in this chapter. Thus nanostructures can serve as potential candidates for FE and FETE based devices due to their geometrical field enhancement effects. In addition, such nanostructures can result in reduced space charge effects compared to flat surfaces even in the absence of any external electric field. 


\subsubsection{Carbon nanostructures}

In addition to metals. different carbon nanostructures were also recently studied extensively for thermionic power generation due to their large geometrical aspect ratio, specific electronic properties, and chemical inertness. However, most carbon nanostructures including pristine $\mathrm{MWNT}^{27}$ and graphitic carbon nanofibers ${ }^{3}$ have shown to exhibit large work functions, in the range of $4-5 \mathrm{eV}$. The work function values of SWNT intercalated with cesium ${ }^{28}$ and potassium ${ }^{29}$ were reduced by $1-2 \mathrm{eV}$. However, thermionic emission testing of these materials was not reported. Thermionic electron emission distribution (TEED) from graphitic nanofibers intercalated with potassium revealed a reduced work function of $2.2 \mathrm{eV}$ at $600-700{ }^{\circ} \mathrm{C}$, but the long term stability of the intercalated atoms could not be evaluated ${ }^{30}$. In another case, photo and thermionic emission from potassium intercalated CNTs was shown to reduce the work function to about $2.0 \mathrm{eV}$. However. the desorption of the potassium atoms at temperatures above $570{ }^{\circ} \mathrm{K}$ led to larger work functions, and very low emission currents resulting from substantial scattering of photo excited electrons was observed ${ }^{31}$. Though work function values were reduced to around $2 \mathrm{eV}$ using barium strontium oxide coatings on CNTs, the operating temperature was very high around $1437^{\circ} \mathrm{K}{ }^{32}$. Also, the stability of the coating at very high temperatures over a period of time was not studied.

Conical carbon nanostructures (CCNTs) with superior structural features have never been explored for thermionic emission. These conical structures, with exposed graphitic edge planes on the entire surface and higher geometrical field enhancement effects might be expected to exhibit enhanced emission characteristics compared to traditional CNTs. In addition, doping into these conical structures with high density of 
graphitic edge planes can be expected to have fundamentally interesting consequences towards altering their electronic properties.

\subsubsection{Diamond}

As mentioned earlier in the Introduction, diamond possesses several unique characteristics features compared to other semiconductors and carbon structures ${ }^{10,11}$ that make it the most ideal material for vacuum electronics and especially for thermionic emission applications. Along with these exceptional properties, the work function values of diamond can be lowered either by surface modification/surface coatings of elements that induce dipole moments and affect the electron affinity or by doping with appropriate dopants causing a change in the position of the Fermi level as (explained below).

(a) Surface modifications: Diamond grown using the chemical vapor deposition (CVD) method has shown to exhibit negative electron affinity (NEA) due to hydrogen termination on the surface ${ }^{13}$. The electron affinity which can also be altered by various surface modification/coatings in turn alters the work function. Some of them include coatings of various metals like $\mathrm{Ni}^{15}, \mathrm{Ta}^{33}$, alkali metals such as $\mathrm{K}^{16}$, alkali metal oxides such as cesium oxides ${ }^{34}$ and fluorides of lithium ${ }^{35}$ and rubidium ${ }^{36}$. Though a change in work function by introduction of NEA was observed, most of the metals were not highly electropositive resulting in weak surface dipole ${ }^{36}$. Moreover, the unstability of these coatings at high temperatures could also prevent their use as thermionic emitter. Thus surface modifications were not explored for thermionic applications. On the other hand, 
work function can also be altered by varying the position of the Fermi level by doping with appropriate dopants which is described below.

(b) n-type doping: p-type doping into diamond using boron has been studied extensively for electronic and biological applications. Higher mobilities and conductivity values have been shown to improve the performance of the applications. However, boron doping introduces an acceptor level at $0.37 \mathrm{eV}$ above the valence band in the diamond ${ }^{37}$. This results in large work function values as the Fermi level was brought closer to valence band, which makes it not suitable for the thermionic applications.

On the other hand n-type doping can introduce a shallow donor level close to the conduction band thus lowering the work function. However, n-type doping into diamond is limited and has been very challenging due to limited or no incorporation of dopants in to bulk diamond lattice. Several n-type dopants such as nitrogen $(\mathrm{N})$, phosphorus (P), sulphur (S) have been studied earlier ${ }^{14}$. Nitrogen has been shown to create deep donor levels at $1.7 \mathrm{eV}$ below the conduction band minimum due to its structural distortion in diamond lattice ${ }^{38}$. Consequently, N-doped diamond does not yield room temperature conductivity, further limiting its electronic feasibility. Sulfur (S) doping into diamond has not been shown to exhibit shallow donor levels even though theoretical modeling predicts it. There is still controversy over the type of donor level (swallow or deep) created by $\mathrm{S}^{14}$.

(c) Co-doping: Another alternative for obtaining the n-type doping in diamond is codoping using different dopant atoms. Co-doping of sulfur and boron has shown to exhibit n-type conductivity, but no significant reduction in the work function was observed ${ }^{39}$. 
Similarly, co-doping of boron and phosphorus in diamond using ion implantation was shown to exhibit $\mathrm{n}$-type conductivity with higher hall mobilities and conductivity values than P-doped diamond films ${ }^{40}$. This was attributed to diamond films with more compatible lattice structure by co-doping than doping with phosphorus alone which has shown to introduce lattice mismatch ${ }^{17}$. However, the work function values were not detected in any of the above mentioned reports. Several theoretical works have been reported for introducing shallow donor levels by co-doping of different elements such as substitutional arsenic and antimony ${ }^{41}$, interstitial lithium and sodium ${ }^{42}$ and phosphorus and sulphur ${ }^{43}$, however lack of experimental evidence limits their validity.

(d) Phosphorus: Phosphorus in single crystalline diamond (111) is considered to be one of the best shallow donors to date, introducing a donor level $0.6 \mathrm{ev}$ below the $\mathrm{CB}^{44}$. It is predicted that, in P-doped diamond, electrons can be easily extracted from the space charge laye ${ }^{45}$ because of ionized donors and creation of defects during P incorporation, which eases the movement of electrons to be extracted ${ }^{46}$. However, some of following factors have limited the successful/controlled doping of satisfactory levels of phosphorus into the diamond: 1 . The difference in covalent bond length between phosphorus $(1.10 \mathrm{~A})$ and carbon $(0.77 \mathrm{~A})$ causes degradation of site symmetry and structural distortion due to the incorporation of phosphorus atoms into the substitutional site. 2. Another challenge arising from the P-C size mismatch that there could be some native defects and structural displacement accompanying the phosphorus incorporation. 3. P incorporation is more favorable on (111), compared to (100) surface which offers several advantages for electronic applications due to ease in achieving smooth surfaces and better electrical and 
optical performances over (111) substrates ${ }^{17}$. Though $\mathrm{P}$ doping into single crystal diamond ${ }^{17.44}$ and diamond films ${ }^{47}$ has been successfully achieved, the thermionic emission characteristics or work function measurements were not explored.

(e) Thermionic emission from n-type diamond: As mentioned earlier, thermionic emission applications require work functions as low as possible $(\phi<2 \mathrm{eV})$ for efficient conversion of waste heat to electricity starting at the temperature as low as $500{ }^{\circ} \mathrm{K}$. Thermionic measurements from surface terminated undoped diamond films has been shown to exhibit work function value of $3.3 \mathrm{eV}^{48,4}$. Sulfur doped nanocrystalline diamond films have shown to exhibit work function of $2.5 \mathrm{eV}^{49}$. But, thermionic emission from nitrogen doped homoepitaxial diamond $d^{50}$, nano ${ }^{51}$ and ultrananocrystalline diamond ${ }^{52}$ films resulted in observed work functions as low as $1.3 \mathrm{eV}$. Recently, work function value as low as $0.9 \mathrm{eV}$ was observed for the phosphorus doped diamond film ${ }^{53}$. However, all the above results were limited to either large single crystals or films consisting grain boundaries which prevented the study of thermionic emission from a truly nanoscale diamond. Towards this goal, thermionic emission from phosphorus doped individual diamond crystals on conical carbon nanostrucutres would be expected to result in improved electron emission properties from reduced space charge effects and lower work functions from enhanced incorporation of dopants in nanocrystals. The following table summarizes all the above discussed cathode materials. 
Table 2.1 List of different thermionic emitter materials with respective work function and operating temperatures

\begin{tabular}{|c|c|c|c|c|}
\hline Class & Cathode Material & $\Phi(\mathrm{eV})$ & $\mathrm{T}(\mathrm{K})$ & Ref \\
\hline \multirow[t]{3}{*}{ Metals } & Tungsten & 4.6 & 2500 & 7 \\
\hline & Tantalum & $4.3-4.4$ & $1500-1800$ & 54 \\
\hline & Rhenium & 5.1 & $1900-2700$ & 55 \\
\hline \multirow[t]{7}{*}{ Metals/Coatings } & Barium on Tungsten & 2 & $1200-1400$ & 7 \\
\hline & Thorium on Tungsten & 2.7 & 2000 & 7 \\
\hline & Cesium on Tungsten & 1.5 & - & 7 \\
\hline & Cesium on Molybdenum & 1.6 & $1000-2000$ & 20 \\
\hline & Thorium oxideTungsten & 2.7 & 2000 & 7 \\
\hline & $\begin{array}{c}\text { Barium strontium oxide } \\
\text { on Tungsten }\end{array}$ & 1.5 & 1000 & 7 \\
\hline & $\begin{array}{c}\text { Gadolinia and Yttria on } \\
\text { Tungsten }\end{array}$ & 2.8 & 1500 & 21 \\
\hline \multirow[t]{4}{*}{$\begin{array}{l}\text { Refractory } \\
\text { compounds }\end{array}$} & $\begin{array}{c}\text { Uranium and Zircnium } \\
\text { carbides }\end{array}$ & $3.1-3.2$ & 2000 & 7 \\
\hline & $\begin{array}{l}\text { Lanthanum hexaboride } \\
\qquad\left(\mathrm{LaB}_{6}\right)\end{array}$ & 2.8 & 1400 & 22 \\
\hline & $\mathrm{LaB}_{6}$ on Tantalum & $2.6-2.8$ & $1173-1573$ & 23 \\
\hline & $\mathrm{LaB}_{6}$ on Tungsten & $2.4-2.6$ & $1000-1400$ & 24 \\
\hline $\begin{array}{c}\text { Carbon } \\
\text { Nanotubes/coatings }\end{array}$ & MWNT/SWNT & $4.5-4.8$ & $1500-2200$ & 27 \\
\hline
\end{tabular}




\begin{tabular}{|c|c|c|c|c|}
\hline & Graphitic nanofibers & $4.2-4.9$ & $900-1000$ & 3 \\
\hline & Potasium intercalated & 2 & 600 & 31 \\
& MWNTs & & & \\
\hline & $\begin{array}{c}\text { Potasium intercaled } \\
\text { graphic nanofiber }\end{array}$ & 2.2 & $870-1070$ & 30 \\
& $\begin{array}{c}\text { Barium strontium oxide } \\
\text { on CNTs }\end{array}$ & 2 & 1437 & 32 \\
\hline Diamond/doping & Undoped nanocrystalline & 3.3 & $973-1173$ & 48 \\
& diamond & & & \\
\hline & Sulfur doped diamond & 2.5 & $900-1100$ & 49 \\
\hline & Nitrogen doped diamond & $1.3-2.4$ & $500-820$ & 50,26 \\
& Phosphorus doped & $0.9-1.18$ & $650-1030$ & 53,26 \\
& diamond & & & \\
\hline
\end{tabular}

\subsection{Field emission}

Field emission (FE) is the phenomena in which the electrons from the surface of conduction band of a solid material tunnel into vacuum under the influence of an external electric field. Fowler, Nordheim, and others have explained this phenomenon on the basis of quantum mechanical tunneling. Under thermal equilibrium, the electrons in the conductor are confined by a potential well and does not have sufficient energy to escape the potential barrier (the minimum energy required also called as work function) into the vacuum. However, the application of an external field results in the bending and thinning 
of the surface potential barrier at the metal vacuum interface as shown in the Figure 2.1 . The work functions $\phi_{\mathrm{eff}}$ and $\phi$ are the minimum energies required to be overcome by the electrons to reach vacuum with and without the presence of electric field respectively. As the barrier lowers due to the applied field, the tunneling distance also decreases resulting in easy penetration of the electrons through this new potential barrier. The shape of the potential barrier depends on the applied electric field, i.e. higher the field applied, the lower the energy barrier. Since FE can be obtained at lower temperatures compared to thermionic emission, it is also referred as cold cathode emission. Typical fields applied can be up to thousands of volts/microns. $\mathrm{FE}$ also requires higher vacuum levels compared to TE for achieving higher efficiency. However, use of nanostructures with high aspect ratios resulting in greater field enhancement effects can offer potential advantages for FE applications.

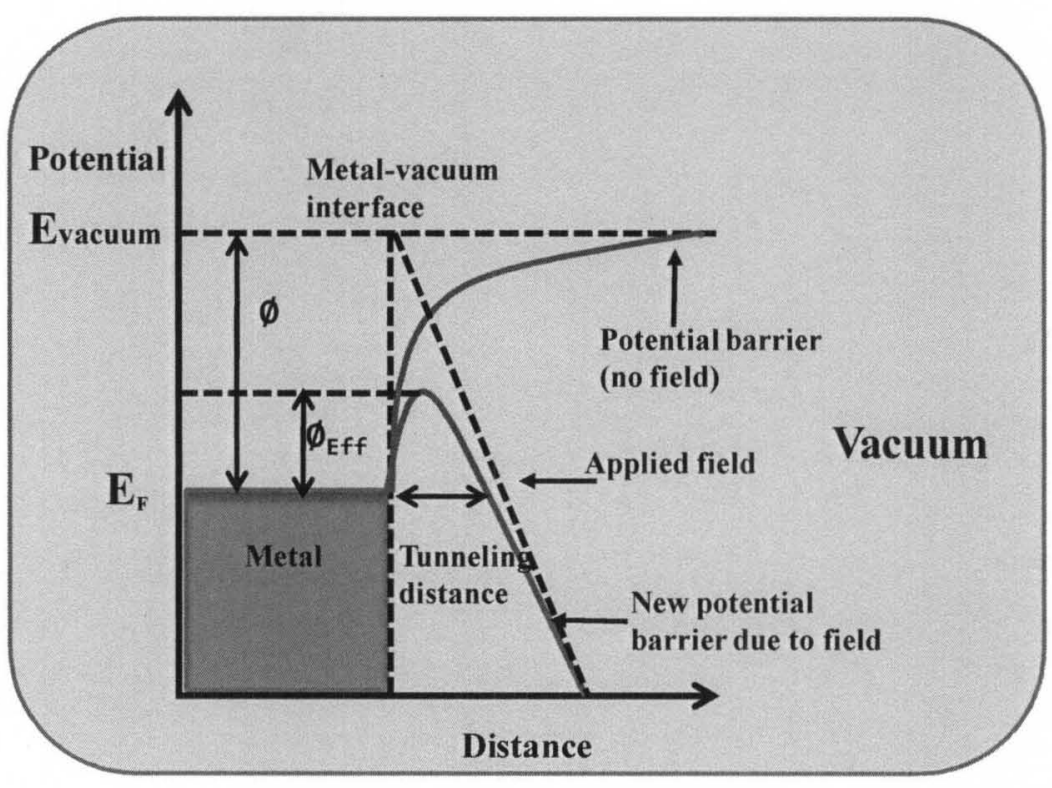

Figure 2.1 Schematic representation band energy diagram for electron emission from a metal with and without the application of external electric field. 
FE emission can be described with simplified Folwer-Nordheim (F-N) equation as shown below

$$
J=\frac{\mathrm{AE}_{a}^{2} \beta^{2} e^{\left[\frac{-\mathrm{B} \phi^{3 / 2}}{\beta E_{a}}\right]}}{\Phi}
$$

Where, $J$ is the emission current density in Amps $/ \mathrm{cm}^{2}, E_{a}$ is the applied electric field, $\phi$ is the work function of the emitter in electron volts $(\mathrm{eV})$, constants $\mathrm{A}=1.56 \times 10^{-6}$ $\mathrm{A} \mathrm{V} \mathrm{V}^{-2} \mathrm{eV}$, and $B=6.83 \times 10^{9} \mathrm{~V}(\mathrm{eV})^{-3 / 2} \mathrm{~m}^{-1}$ and dimensionless parameter $\beta$ is the field enhancement factor used as a measure for the enhancement (amplification) of the applied electric field at the apex of the emitter and to describe the emitter's performance given as

$$
\beta=E_{e f f} / E_{a}
$$

Where, $E_{\text {eff }}$ is the effective electric field at the tip of the emitter, and $E_{a}$ is applied electric field. The effect of the enhancement of the field can be visualized as lowering of the potential barrier and decrease in the tunneling distance. The higher the value of $\beta$, the higher the enhancement of the applied electric field, lowering the value of the applied electric field for achieving significant emission. Electric field enhancement can be an indication of the effect due to the geometry structure of the emitters and generally can be expressed as $\beta=\mathrm{h} / \gamma$, where $\mathrm{h}$ is the height of an emitter and $\gamma$ is the curvature of the emitting surface ${ }^{56}$. Thus compared to flat surface of the emitter, sharp nanostructures with high aspect ratios such as CCNTs can enhance the field to a greater extent and thus lowering the required applied electric fields for the emission, described in detail in the results chapter. However, in the case of an array of emitters, field screening effect 
resulting from the neighboring emitters decreases the expected field amplification at the tip and hampering the $\mathrm{FE}$ characteristics ${ }^{57}$.

(a) Field emission characteristics of carbon nanostructures: Field emission from carbon nanotubes (CNTs) has been explored for potential applications varying from flat panel displays to miniature scanning electron microscope columns ${ }^{58}$. CNTs are known to possess most of the favorable properties as field emitters such as high aspect ratio, good mechanical, electrical, thermal and chemical properties, and ability to be grown as vertical arrays with controlled density ${ }^{59,60}$. The dependence of field emission properties of CNTs on (i) diameter (ii) length (iii) density/sparsity (spacing between neighboring tubes) (iv) alignment/randomness, (v) wall defects, and (vi) surface absorbates has been explored to a greater depth ${ }^{61}$. Higher values of $\beta$ result in higher local electric field (low turn on fields) as well as current densities, which is extremely desirable in many applications such as X-ray devices. Although higher $\beta$ values $(2,500-10,000)$ and lower turn-on fields $(0.8-1.5 \mathrm{~V} / \mu \mathrm{m})$ have been reported for single walled nanotubes (SWNTs) ${ }^{62}$ and multi walled nanotubes (MWNTs) ${ }^{63}$, the lower current densities $\left(10 \mathrm{~mA} / \mathrm{cm}^{2}\right)$ limit their applicability as efficient cold cathodes for applications in electron and X-ray sources. Considering these limitations, new morphological structures with superior field emission properties are highly desired.

CCNTs, essentially consisting of central carbon nanotube surrounded by helical graphene sheets, exhibit the stability of a cone combined with the advantage of a cylindrical morphology for the field emission at low electric fields. Long CCNTs grown vertically with moderate densities, together with their tapered morphologies can be well 
separated at their tips, which in turn contribute to the reduction of field screening effect provoked by the proximity of neighboring structures when the arrays are used as field emitters. In fact, the inter-emitter distance should be at least 1-2 times the emitter length

to minimize the field screening effect ${ }^{57}$. Conical structure with its high-aspect ratio (micron scale base to nanometer scale tip) is expected to be mechanically and thermally more stable compared to a constant diameter nanotube (narrow cylinder) of the same tip diameter. Also tapered structures, due to their increasing cross sectional area away from the tip, increase the thermal transport and are likely to sustain greater current densities than nanotubes. Moreover, conical structures offer greater rigidity and ease of operation in mounting for use as tips for scanning probe microscopy than carbon nanotubes being used currently. Field emission measurements on CCNTs grown with metal catalysts have been reported ${ }^{64}$. However, the extent of the above studies is limited and the structures studied do not represent the ideal conical morphology due to the presence of metal contamination at their tip. Thus the study of field emission characteristics of an ideal conical morphological structures involving CCNT array samples with varying aspect ratio, density and wall structures would be expected to result in enhanced emission properties compared to CNTs.

\subsection{Conical carbon nanotubes}

Since the discovery of the carbon nanotubes (CNTs) by IIjima ${ }^{65}$, intense research has been devoted on the controlled growth and perspective applications of CNTs in different areas. Exciting progress has been reported for the use of CNTs in fabrication of nanodevices, scanning probe microscopy, field emitters and manipulating devices. 
However, the use of CNTs for all these applications involves exhaustive processes with complex and expensive tools. On the other hand, a cone-shaped carbon nanostructure combined with its mechanical rigidity and ease of operation can be attractive for practical applications including nanoscale devices, scanning microscopy, atomic force microscopy, field emission devices, drug delivery. Conical carbon nanostructures, exhibiting novel morphological forms of one-dimensional carbon nanostructures, present a number of interesting characteristics compared to multiwalled carbon nanotubes (MWNTs). Different forms of conical carbon structures include graphitic cones ${ }^{66}$, conical crystals of graphite $^{67}$, carbon nanopipette ${ }^{68}$, tubular graphite cones ${ }^{69}$, carbon nanocones ${ }^{70}$, tubular carbon cones ${ }^{64}$, conical carbon filaments ${ }^{71,72}$, conical carbon fiber ${ }^{73}$, nanohorns ${ }^{74}$. Of all these various forms of conical carbon structures, the so-called "carbon nanopipettes" 68 or "conical carbon nanotubes" (CCNT) are more interesting due to their unique features including tapering geometry from micron scale at base to nanoscale at tip and presence of high density of graphitic edge planes surrounding the central hallow core. Such conical carbon nanotube arrays can easily be converted to nanoelectrode ensembles ${ }^{75}$, and have exhibited high sensitivity toward detection of neurotransmitters such as dopamine due to the presence of very reactive graphitic edge planes along the entire surface. Also these structures with high mechanical and good electrical contact with substrate can support larger current densities and exhibit superior field emission characteristics compared to multi-walled carbon nanotubes reported to date ${ }^{76}$, explained in detail in later section. These structures with open tips and long aspect ratios are also of probable interest toward drug delivery applications ${ }^{77}$. 
However, the synthesis of these conical structures was limited to Fe, $\mathrm{Pt}$ or Au needles which are vertically immersed into microwave plasma $^{68,69}$ preventing their use for practical applications owing to small area and cylindrical geometry of the wire substrate. It is thus necessary to obtain these CCNT arrays over flat substrates to understand their characteristic behavior and for the ease of application. Though the control over the

structural properties of CCNTs on wire substrate was achieved ${ }^{73,76}$, several aspects about the actual prevailing conditions for nucleation and growth have not been clearly understood and thus it has been difficult to replicate the growth parameters such as radical species concentration and temperature that existed at the wires immersed in plasma discharges. However, the synthesis of these structures on planar steel substrates ${ }^{78}$, silicon substrate ${ }^{79}$ was recently reported with limited understanding on the reproducibility of such experiments as they typically result in MWNTs.

\subsubsection{Growth mechanisms}

To date, several mechanisms were proposed for the observed evolution of conical morphology with inadequate consistency among one another. For example, a mechanism of shrinking catalyst along with simultaneous growth of graphene layers was proposed to be responsible for the conical growth of tubular carbon cones ${ }^{64}$. It was proposed that, a catalyst assisted tip led growth of central nanotube was further surrounded by helical sheets of graphite coiling around giving rise to a conical geometry as a result of competition between the growth and etching. A change in the size of the catalyst particle during the growth was also attributed to be another crucial factor for resulting conical morphology due to change in nanotube diameter with shrinking in catalyst size as shown 
in the Figur 2.2. However, there was no evidence either for shrinking catalyst or for the change of the nanotube diameter.

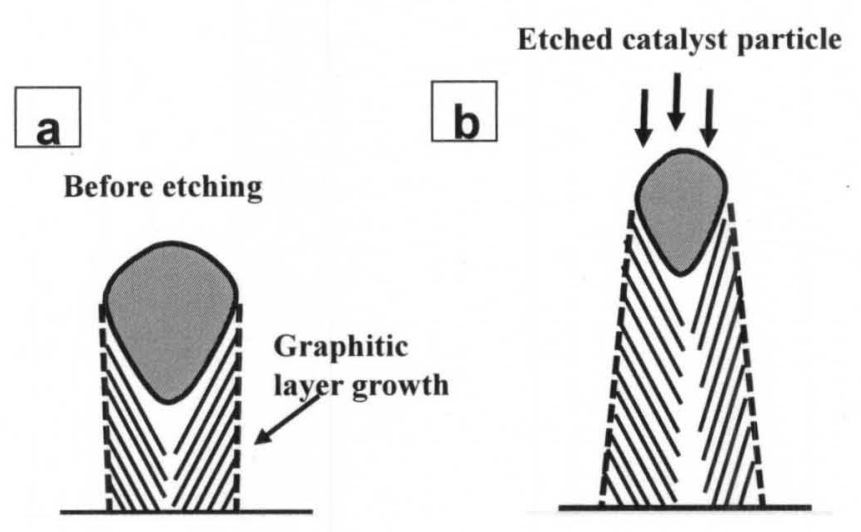

Figure 2.2 Schematic of (a) nucleation and growth of initial nanotube before etching of the catalyst (b) formation of conical morphology resulting from shrinking of the catalyst after being etched.

In the case of tubular graphite cone $e^{78}$, the presence of a large catalyst particle at the base and its role in nucleation of new graphene layers was attributed to the growth of conical structure. The increasing size of the catalyst (from the substrate) along with simultaneous growth of coaxial and radial directional growth of graphite sheets from this catalyst was suggested to be responsible for the evolution of conical geometry as shown in the Figure 2.3. However, clear evidence of increasing catalyst could not be provided. Moreover, though a continuous supply of the catalyst was claimed to be necessary for the growth, similar structures were synthesized using limited catalyst ${ }^{79}$ (using a thin layer of catalyst on the substrate), with the actual role of catalyst not apparent. Similarly in the case of conical carbon filaments ${ }^{71}$, a base-growth mechanism along with delayed nucleation of graphene sheets by continuous segregation of dissolved carbon atoms, together with deposition of amorphous carbon on catalyst was proposed to be responsible 
for the formation of the conical morphology. But the presence of catalyst at the base could not be evidently shown.
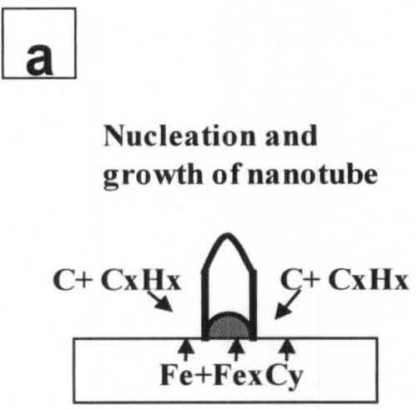

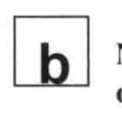

Nucleation and growth of graphitic sheets

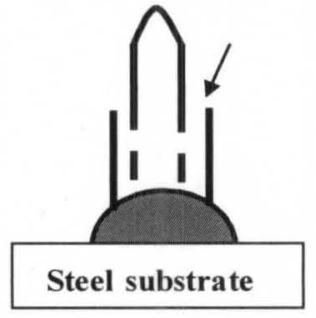

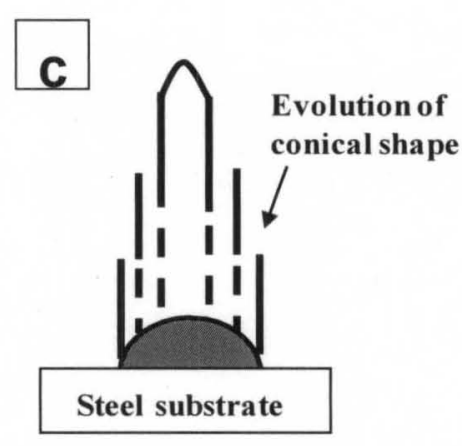

Figure 2.3 Schematic illustration of a catalyst-assisted basal growth of a central carbon nanotube followed by continuous nucleation of new graphene walls for thickening the base while tapering vertically in thickness forming a conical structure.

In case of carbon nanopipettes ${ }^{68}$, a tip led growth of central nanotube followed by growth and simultaneous etching of the helical graphene sheets coiling around the nanotube was assumed to be responsible for the observed conical shape as seen in Figure 2.4. Though the catalyst particle at the tip was assumed to be evaporated during the growth, clear evidence of absence of catalyst at the base could not be demonstrated. The variation of rate of etching Vs deposition depending on the position of the substrate inside the reactor was accounted for the observed variation in the aspect ratio of structures. Slower etching near the tip in the plasma leading to low aspect ratio (Figure 2.4b) and faster etching rate away from the tip leading to longer aspect ratio (Figure 2.4c) as shown below. 

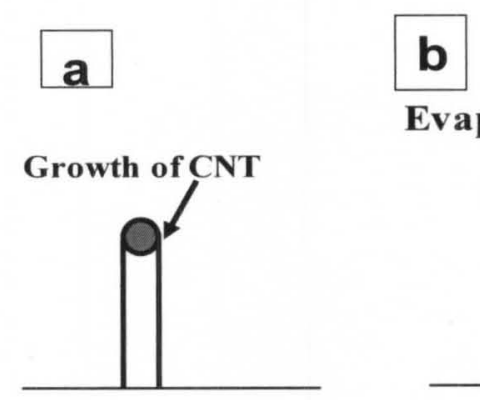

Evaporation of catalyst

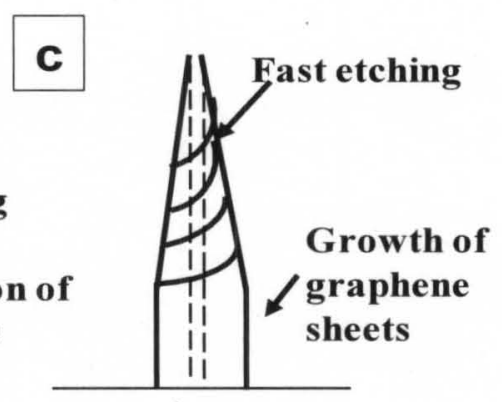

Figure 2.4 Schematic illustration of catalyst assisted tip-led growth of a central carbon nanotube followed by the evaporation of the catalyst particle and simultaneous deposition and etching of the helical graphite edge planes leading to the conical morphology.

In the case of carbon nanocone ${ }^{70}$, a tip led growth of central nanotube associated with simultaneous two directional growth surrounding the nanotube was attributed to be responsible for the resulting conical geometry. Vertical catalyst growth via diffusion of carbon through the catalyst particle and lateral growth by precipitation of carbon from the discharge at the outer walls consequently resulted in conical structure as sown in Figure 2.5. Similar mechanism of tip led growth along with the precipitation of carbon was also reported in the case of conical carbon fiber $^{73}$. However, the role of hydrogen as etchant was attributed as the crucial factor for evolution of conical morphology. These two structures are probably amorphous in nature (no evidence of cryastlline structure) compared to crystalline graphitic structures presented in other studies ${ }^{68,69}$ as a result of precipitation of carbon on the surface and do not represent the ideal conical morphology that is of interest in this context. 

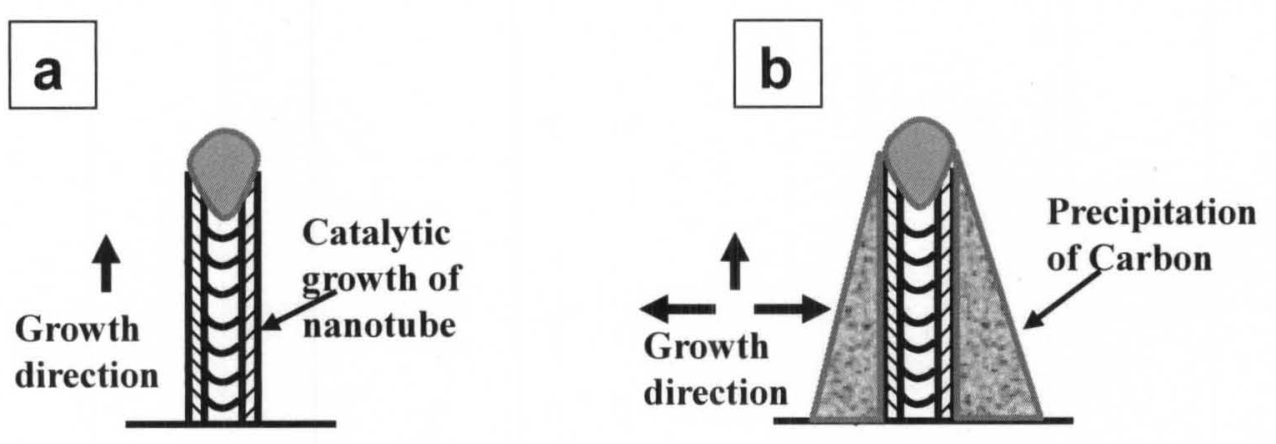

Figure 2.5 Schematic representation of (a) tip led growth of vertically aligned carbon nanotube (b) evolution of conical morphology due to additional precipitation of carbon on the outer wall of the inner nanotube

In the absence of consistency in the suggested mechanisms, several questions regarding the growth of these conical structures still remain unanswered. The two most important questions are: (1) Does the catalyst play any role in the conical growth apart from the nucleation of the inner multiwalled nanotube?; (2) If so, where is the catalyst present (tip or base) and what is responsible for the growth and formation of the new graphene layers? Hence, in this study, a detailed investigation on the nucleation and growth of CCNTs using different foil substrates, different catalyst metals and timeresolved growth kinetics and short-duration experiments to gain insight into the role of catalyst and the possible route for conical shape evolution was undertaken.

\subsection{Diamond nucleation on carbon nanostructures}

The conical carbon tubular structures are particularly interesting for studying diamond nucleation as their surfaces consist of a high density of edge-plane sites. Furthermore, the conical structures could serve as good templates for producing nanodiamond tip arrays, which could be made into diamond nanoelectrode ensembles 
easily by the simple polymer-masking technique described in our earlier work ${ }^{75}$. Also, nanodiamond tip arrays could find interest in functionalized biosensor ${ }^{80}$ and field emission $^{81}$ and thermionic emission applications as described in the earlier chapter. Furthermore, there is an increased interest in synthesizing diamond-based, onedimensional structures and their use in high-temperature diodes and composites ${ }^{82,83}$.

Some of the earlier work on the nucleation of diamond on graphitic/carbon nanostructures reported to be exhibiting different mechanistic approaches as described below. Theoretical and experimental studies provided evidence that the nucleation may be mediated through hydrogenation of graphite edge plane ${ }^{84,85,86}$. Recent experiments involving hydrogen exposure to MWNT structures yielded a high density of diamond nucleation throughout the MWNT while destroying the wall structures ${ }^{87}$. The same experiments performed over a longer duration resulted in the anisotropic growth of short diamond nanorods from the diamond crystals ${ }^{88}$. These studies suggest that the nucleation occurs via the formation of amorphous carbon ${ }^{89,90}$. In other studies using an external carbon source in a hot filament (HF)CVD reactor, SWCNTs were uniformly coated with nanodiamond $^{91,92}$. In the case of diamond nucleation onto SWCNTs, it was proposed that the atomic hydrogen creates the necessary defect for subsequent hydrogenation to form nuclei for nanocrystalline diamond ${ }^{92}$. It was also reported that carbon can exist in bodycentered cubic (bcc), face-centered cubic (fcc), and other phases at the nanometer scale ${ }^{93}$ which may act as intermediates for diamond nucleation, although this has not been confirmed. Despite much progress through prior studies by a number of investigators, the pathway for diamond nucleation is not completely understood. In order to understand the 
nucleation process further, we investigated hydrogenation of the conical carbon tubular structures (CCNTs) containing surfaces with a high density of edge-plane sites.

\subsection{Summary}

The work function values of different materials, including metals and various carbon based nanostructures, were shown to be lowered using several different surface treatments. However, very limited success was reported due to problems associated with stability and compatibility of these coatings. Recently, some progress was achieved with n-type diamond films. However, successful phosphorus doping in diamond has been very challenging, and there is no information on P-doping in a true diamond crystal free from grain boundaries. Studying phosphorus doping in individual diamond nanocrystals could result in better understanding of doping and reduced work function values with enhanced conductivity.

CCNTs represent new structures with exposed graphitic edge plane sites on the surface. The properties of these structures are not understood for various applications. One of the main challenges is to grow these structures on a flat substrate in large area with controlled aspect ratios and densities and to understand the underlining nucleation and growth mechanism. Field and thermionic emission characteristics from CNTs has been studied extensively; however, study of emission properties of these novel CCNTs with improved characteristic features might result in enhanced emission characteristics

Diamond nucleation studies on MWNT and SWNT structures have been successfully reported. But the pathway for nucleation is not completely understood. In addition, study of diamond nucleation on CCNTs exhibiting unique morphological 
characteristics compared to MWNT or SWNT has never been investigated. Thus, the study of the underlining nucleation and growth mechanism of diamond nanocrystals on CCNTs may yield new hybrid architectures with control over sizes and density for various applications including field and thermionic emission. 


\section{CHAPTER 3}

\section{EXPERIMENTAL PROCEDURES}

\subsection{Introduction}

As described in earlier chapters, CCNTs exhibiting superior characteristic features compared to CNT were mainly limited due to their synthesis on wire substrates having small areas ${ }^{67}$. To overcome this problem a new experimental set up was demonstrated in this chapter for large area growth of CCNTs. Initial experiments for the growth of CCNTs on platinum wires and modification using graphite, and tungsten foil substrates are described. Also, the experimental procedures used for studying the nucleation, growth and phosphorus doping of diamond nanocrystals, and diamond films are presented.

As mentioned in previous chapter, n-type doping especially using phosphorus can result in obtaining low work function values towards thermal energy conversion ${ }^{16}$. However, a gas phase source employed in most of the earlier works is very toxic and requires stringent operating procedures and supervision. On the contrary, organic precursors are much less toxic and moderately easy to operate ${ }^{94}$. The experimental set up for employing Di-tert-butyl phosphine (TBP) precursor and its vapor pressure determination procedures are demonstrated here. Later the experimental procedure for growth and phosphorus doping of diamond crystals on these CCNT and diamond films is discussed. 
Characterization of such various nanostructures could not be achieved using just one or two techniques, but involved several characterization tools. Morphological characterization was accomplished by scanning electron microscopy (SEM). Transmission electron miscroscopy (TEM) was used for in depth study of the morphology and crystal structure of diamond and carbon nano structures. The quality of the diamond crystals was verified with the help of Raman spectroscopy. Surface characterization was achieved using Ultraviolet photospectroscopy (UPS) for the study of the Fermi level and work function of CCNTs and doped and undoped diamond films. Finally, the details of the experimental set ups of field and thermionic measurements for the study of emission characteristics and determination of work function are described.

\subsection{Nucleation and growth experiments}

CCNT arrays on platinum wire substrates were synthesis using ASTeX 5010 model microwave plasma chemical vapor deposition (MW CVD) reactor. The reactor sketch is shown in Figure 3.1. This reactor is equipped with microwave generator (power supply) with $1.5 \mathrm{~kW}$. The micro waves generated are transmitted through the reactor's waveguide. The waveguide has a 3 -stub tuner that prevents reflected microwaves from returning to the power supply, which can damage it. The microwaves are transmitted into the reactor chamber consisting of a quartz bell jar in which the plasma is generated through the ionization of neutral gas by the high frequency electric fields transmitted through the waveguide. Plasma in general refers to an electrically neutral medium consisting of positively charged ions and negatively charged electrons as a result of the ionization of a neutral gas. The sufficient energy required for this ionization can be provided by thermal energy or energetic beams. However, the most common method for 
generating and sustaining the plasma at low-temperature is by applying electric field to neutral gas, specially using Microwaves. The interaction of these high-intensity radioactive radiations $(2.45 \mathrm{GHz})$ with neutral gas results in the formation of few electrons and ions resulting in a glow discharge. Plasma is non-isothermal due to difference in the temperatures of ions (heavier) and electrons that are accelerated to very high energy levels. These charged carriers accelerated in these electric fields when collide with atoms and molecules of the gas, new charged particles will be formed. As a result of these collisions in gas phase, the neutral and ionic species formed inside the reactor are responsible for the deposition and growth of the solid material on the substrate. The MWCVD reactor used here is not equipped with an independent heater, but the temperature can be controlled by pressure in the reactor chamber and microwave power employed.

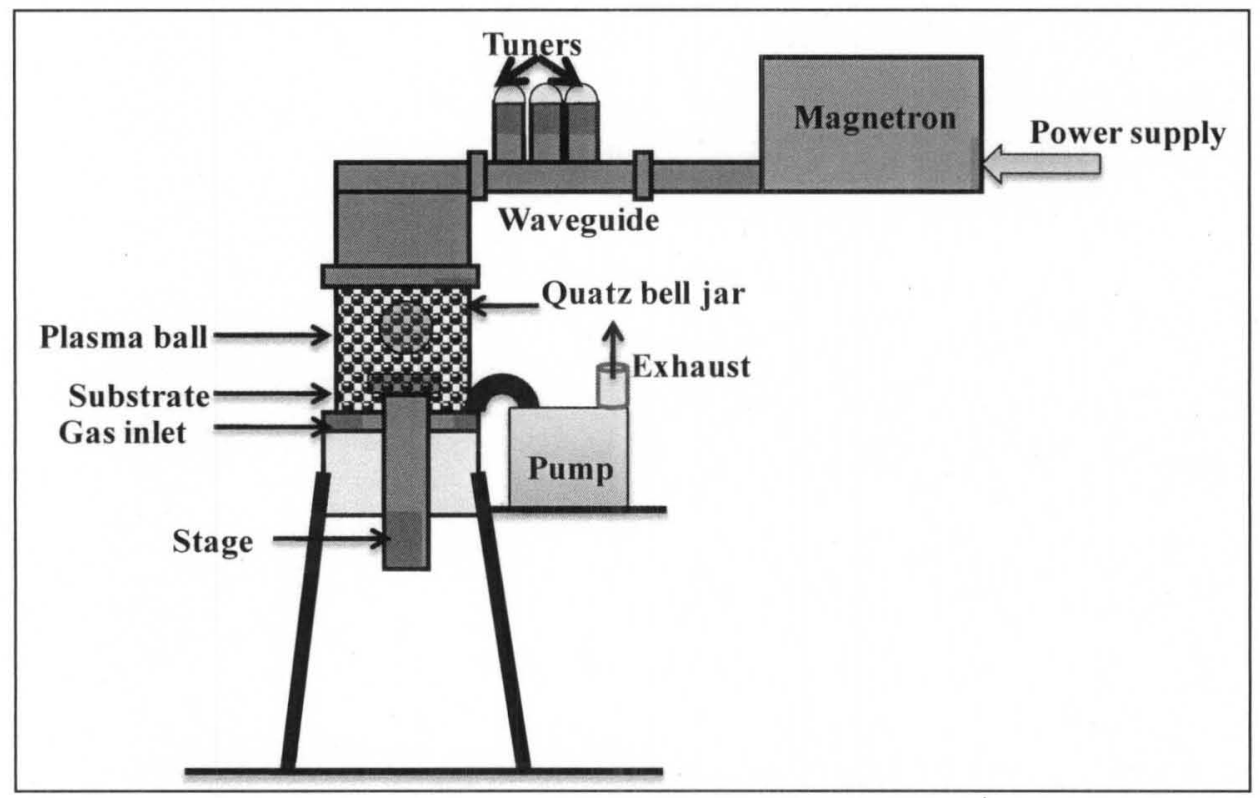

Figure 3.1 Schematic of MWCVD reactor. 
The nucleation and growth experiments of diamond crystals on wire substrates were performed using a SEKI model AX5200S-Electron Cyclotron Resonance (ECR) MWCVD reactor equipped with a radio frequency $(\mathrm{RF})$ induction heating stage. ECR plasma is a typical example of microwave plasma in the presence of magnetic fields. The presence of magnetic field facilitates the ignition of microwave discharges in the lowpressure $\left(1-10^{-3} \mathrm{~Pa}\right)$ regime with a low collision frequency and thus, with absorption of low power ${ }^{94}$. In the presence of magnetic field, the electrons rotate with the electron cyclotron frequency, which when equals to the microwave frequency $(2.54 \mathrm{GHz})$ the power absorption reaches its maximum value. Low operating pressure contributes to a large mean free path length of the electrons, which ensures a high ionization rate of the neutral gas resulting in high density of ions with lower energy (gentler plasma).

\subsubsection{Growth of CCNT using wire substrates}

The growth of CCNT arrays on platinum wire substrates was achieved in similar fashion as reported earlier by Mani et.al. in our group ${ }^{68}$. Platinum wires $(0.3 \mathrm{~mm}$ dia and $2 \mathrm{~cm}$ length) were used as substrates. The platinum wires were cleaned with acetone and a graphite susceptor with multiple holes is used for placing several wires simultaneously. This susceptor is placed on a graphite stage and inserted into the reactor. As shown in the Figure 3.2 , the platinum wires placed vertically were immersed into plasma discharge inside the reactor. The temperature at the tip of these wire substrates is very close to the melting point of the platinum $\left(\sim 2000^{\circ} \mathrm{K}\right)$ as reported earlier. This is also evident from the melting of the platinum wires forming a bulb (ball shape) at the very tip during the initial few seconds after the ignition of the plasma. The reactor was pumped down to few milli torr using a mechanical or roughing pump. The experiments were conducted at 
microwave power of $950 \mathrm{~W}$, pressure of 24 Torr with a methane composition in the range of $1-1.35 \%$ in hydrogen $(200 \mathrm{sccm})$.

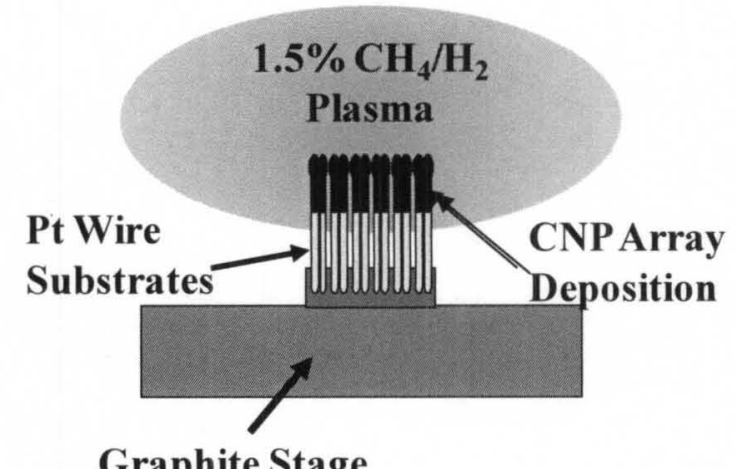

Figure 3.2 Schematic illustration of growth of CCNTs on platinum wire substrates.

\subsubsection{Growth of CCNTs using foil substrates}

Rectangular sheets of graphite and tungsten foils (0.13-0.15 mm thick) were cleaned with acetone and sputtered with platinum to obtain a thin layer of catalyst (10 $\mathrm{nm})$. These foils were rolled around a $1-3 \mathrm{~mm}$ diameter graphite rod and immersed vertically into microwave plasma by graphite susceptor similar to the wire substrates mentioned above. The schematic illustration of the experimental configuration for a single foil rolled into cylinder is shown in Figure 3.3. Using this similar method as above, several foils can be employed simultaneously by utilizing a susceptor with multiple holes. Experiments were conducted at 30-50 torr pressure with microwave power of 900-1000 W using a gas composition of 1-1.5\% methane in hydrogen (total flow rate of $200 \mathrm{sccm}$ ). Several other experiments include using in-situ catalyst, high pressures and etching and regrowth of CCNTs, which are described in detailed in next chapter. After the deposition, the foils can be easily unrolled giving a large area of CCNTs on a flat substrate. 


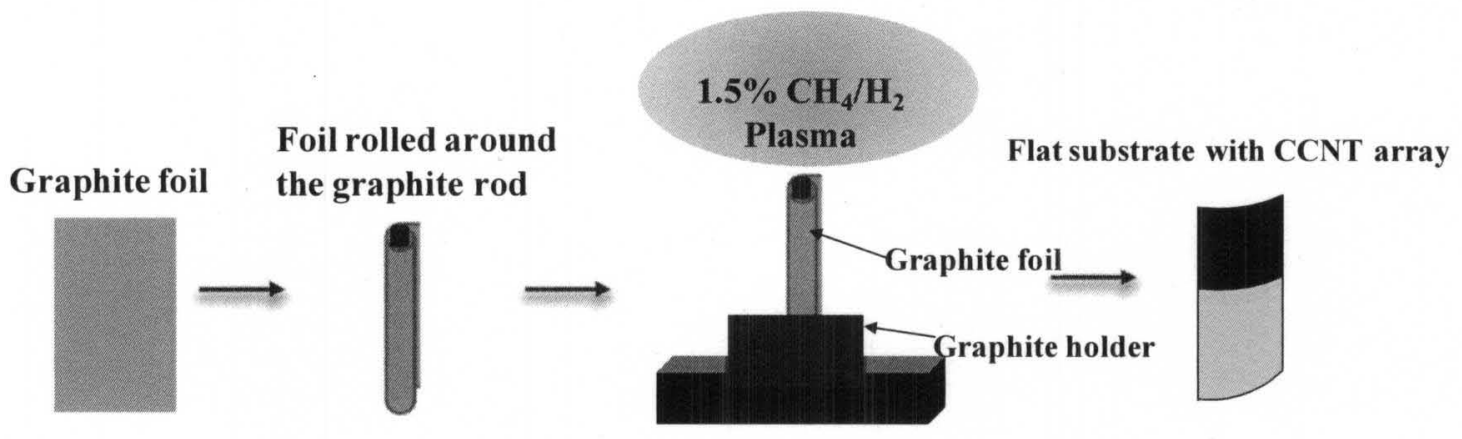

Figure 3.3 Schematic illustration of the series of steps for the synthesis of CCNTs on foil substrates

\subsubsection{Diamond nucleation on CCNTs}

The platinum wires with CCNT array deposition (as discussed above) were used as the substrate for the nucleation and growth of diamond on these CCNTs. As shown in Figure 3.4, the platinum wire was placed horizontally on to a graphite susceptor in an SEKI model AX5200S-ECR MWCVD reactor. Several experiments were conducted using methane composition ranging from $2-5 \%$ in hydrogen $\left(200 \mathrm{sccm} \mathrm{H}_{2}\right)$ using MW power of $900 \mathrm{~W}$ and substrate temperature of $1023 \mathrm{~K}$ at 25 torr pressure. Some experiments were conducted using ECR plasma at lower pressures ranging from 50-100 milli Torr with methane composition ranging from $0-5 \% \mathrm{CH}_{4}$ in $\mathrm{H}_{2}$ using 500 Watts of MW power and a substrate temperature of $1000^{\circ} \mathrm{K}$ for two hours. 


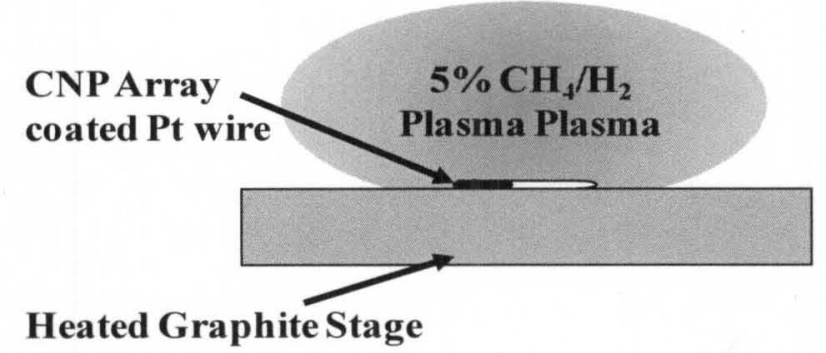

Figure 3.4 Schematic representation of horizontal positioning of the platinum wire coated with CCNT array for diamond nucleation.

\subsection{Organic precursor - Di-tert-butyl phosphine (TBP)}

Generally a gas phase source (hydrides e.g. phosphine) used for phosphorus doping, is very toxic and requires stringent operating procedures and supervision. On contrary, organic precursors are much less toxic and moderately easy to operate. The precursor employed here is Di-tert-butyl phosphine (TBP), which has reported be less two orders of magnitude lesser toxicity compared to $\mathrm{PH} 3^{95}$. Di-tert-butyl phosphine (50g) was obtained in the form of liquid enclosed inside a stain less steel bubbler from Strem Chemicals. The schematic illustration of the entire bubbler set-up (to the reactor model 5010 AsTex MWCVD shown above) can be seen in the Figure 3.5. All the connections and reactor is ensured leak tight using helium leak detector with sensitivity of $5 \times 10^{-12}$ ATM $\mathrm{cc} / \mathrm{sec}$. All the gas lines including the bubbler and the reactor are always under vacuum during the operation, preventing the leakage of the precursor into the atmosphere. Also the entire bubbler set up is enclosed inside a containment vessel as shown in the schematic. The vessel is connected to an exhaust preventing any accidental spread or exposure of organic fumes to both the reactor operator and the surrounding 
bystanders.

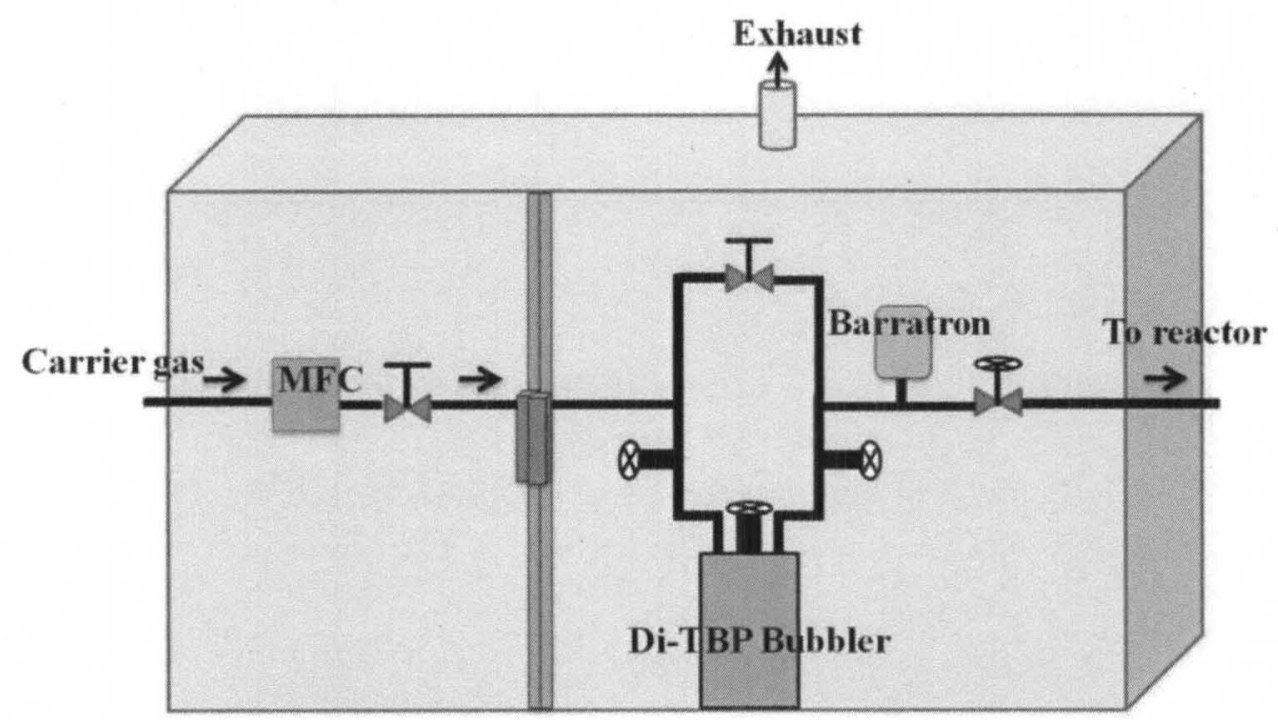

Figure 3.5 Schematic illustration of bubbler set up for phosphorus doping

\subsubsection{Determination of vapor pressure of Di-TBP}

The bubbler was pressurized with an inert gas (argon) under vacuum. The accurate vapor pressure of this chemical was not been reported or known. Thus the determination of the vapor pressure of this liquid precursor at the room temperature was attempted here. As the bubbler cylinder was pressurized with argon gas, it was essential to remove the entire gas before the vapor of the precursor was collected for measuring. This was done using "Freeze and Pause" method described below. In Freeze and Pause method, the bubbler cylinder was placed in a vessel containing solid carbon dioxide to freeze the liquid precursor. Under this condition, the valve of the cylinder was opened to release the argon gas which was pumped down through the reactor. Later it was brought to normal room temperature and maintained at that temperature for few hrs. Then the entire process of removing the gas was repeated for at least 2 more times, to make sure 
the absence of any traces of argon gas in the cylinder. After the elimination of argon gas, the only vapor left inside the reactor at room temperature could be that of the precursor, which was measured to be 1.7 torr.

During the experiment, a carrier gas (hydrogen) was used to carry the vapors of the precursor generated inside the bubbler through the stainless steel lines into the reactor. The pressure of this vapor was measured using the pressure gauge shown in the Figure 3.5. The flow rate of the precursor (concentration) can be controlled by regulating the carrier gas flow rate and total pressure in bubbler line (which can be monitored using the needle valve shown in the schematic). The equation for monitoring the flow rate of the precursor is given below.

$$
F_{P}(\mathrm{~mol} / \mathrm{min})=\frac{P_{V}}{P_{T}} \times V(\mathrm{sccm}) \times \frac{1 \text { mole }}{22414 \mathrm{~cm}^{3}} \quad 3.1
$$

Where $F_{P}$ is the molar flow rate of the precursor, $P_{V}$ and $P_{T}$ are the vapor pressure of the precursor and total pressure of the line respectively and $V$ is the carrier gas flow rate in sccm (standard cubic centimeter per minute). The substrate is supported by a stage inserted into the reactor chamber. Once the plasma is ignited, radicals generated from the decomposition of gases undergo several reactions in the gas phase and simultaneously at the surface of the substrate resulting in the deposition of the desired material. Microwave power, pressure and the gas phase composition can be chosen accordingly by the operator.

\subsubsection{P-doping into diamond crystals on CCNTs}

Graphite foil substrate with as synthesized CCNT arrays as mentioned earlier was used as the substrate for nucleation and growth of diamond crystals. The foil substrate 
was pretreated in ultra-sonication diamond solution bath $(0-1$ micron powder in acetone solution) for few seconds followed by cleaning with acetone. The foil was then immersed vertically into the plasma same as shown in Figure 3.3 for the growth of diamond. The diamond growth experiments were conducted using AsTeX 5010 microwave plasma reactor $(1.5 \mathrm{~kW})$. Di-teriterty butyl phosphine was used as the liquid source for the phosphorus doping. After the reactor was pumped to few milli torrs, hydrogen and methane gases were introduced along with phosphorus precursor which was also introduced using hydrogen carrier gas simultaneously. Once the bubbler pressure was stabilized to the required value, the plasma was ignited and radicals generated from the decomposition of gases undergo several reactions in the gas phase and simultaneously at the surface of the substrate resulting in the deposition of the desired material. Experiments were conducted at microwave power of $900-1000 \mathrm{~W}$, pressures $30-50$ torr with $0.2-1.5 \%$ methane in hydrogen with total flow rate of $200 \mathrm{sccm}$. The phosphorus concentration was varied from $8000-30,000 \mathrm{ppm}$ varying the bubbler pressure, carrier hydrogen gas flow rate. The duration of the experiments lasted from $12 \mathrm{~min}$ to $1 \mathrm{hr}$. The carrier gas flow rate of $4 \mathrm{sccm}$ is used with bubbler pressure of 720 torr.

\subsubsection{P-doping into diamond films}

Silicon wafers (p-type) were used as substrates for the growth of diamond films. The wafer was cleaned with acetone and pretreated in ultra-sonication diamond solution bath mentioned above and cleaned with acetone. Undoped diamond film growth experiments were conducted in AX5200S-ECR MWCVD reactor mentioned earlier. The substrate was placed on the graphite susceptor and placed on the graphite stage. Experimental conditions include $3-5 \%$ methane in hydrogen $(200 \mathrm{sccm})$ at 60 torr, $1450 \mathrm{~W}$ microwave 
power and stage at temperature of $650{ }^{\circ} \mathrm{C}$ for about $10 \mathrm{hrs}$. P-doped diamond films were grown using these undoped diamond films as substrates employing similar experimental procedure described for phosphorus doping of individual diamond crystals in the earlier section. The conditions include $0.2 \%$ methane in hydrogen $(200 \mathrm{sccm})$ at $980 \mathrm{~W}$ microwave power and 50 torr pressure. The carrier gas (hydrogen) flow rate of $4 \mathrm{sccm}$ is used while maintaining the bubbler pressure at 46 torr for about $9 \mathrm{hrs}$.

\subsection{Materials characterization}

The synthesized CCNT and diamond nanostructures were characterized using a number of techniques such as electron microscopy, Raman and Ultra violet photo spectroscopy etc. The morphology of the samples was studied by Scanning electron miscroscopy (FEI Model Nova 600 nano SEM). The crystallinity of the various carbon nanostructures synthesized was studies using a field emission gun Tecnai F20 microscope operated at $200 \mathrm{kV}$. A symmetrical multi-bean illumination was selected for highresolution transmission electron microscopy (HRTEM) imaging of the grids. Some of diamond samples were also characterized using Raman spectroscopy to determine their phase. Other characterization techniques were also employed to determine the work function and electron emission characteristics of the materials used, especially for harvesting waste heat applications. These techniques include ultraviolet photoelectron spectroscopy, field emission and thermionic emission measurements. These techniques are discussed in detail in the following sections.

\subsubsection{Ultraviolet Photoelectron Spectroscopy (UPS)}


Ultra violet Photoelectron spectroscopy is the technique used for the study of the electronic state of the surface region of a sample utilizing photo-ionization and analysis of the kinetic energy distribution of the emitted photoelectrons. The source of exciting radiation in this is the UV radiation (with photon energy of $10-45 \mathrm{eV}$ ). This normally includes a noble gas discharge lamp such as frequently used He-discharge lamp emitting He-I and He-II radiations of energies $21.2 \mathrm{eV}$ and $40.83 \mathrm{eV}$ respectively. Such low energy radiation is only capable of ionizing the electron from the outermost levels of atoms which include the valence band levels. The basic equation governing the working of the UPS is $\boldsymbol{K} \boldsymbol{E}=\boldsymbol{h} \boldsymbol{v}-\boldsymbol{B} \boldsymbol{E}-\Phi_{\mathrm{s}}$, where $K E$ is the kinetic energy of the emitted electrons $(\mathrm{eV}), h$ - Planck constant $\left(6.62 \times 10^{-34} \mathrm{~J} \mathrm{~s}\right), v$ - frequency $(\mathrm{Hz})$ of the radiation $(h v$ is the energy of the UV radiation), BE is the binding energy of the electron (eV) and $\Phi_{s}$ is the work function of the sample (eV). Figure 3.6 shows the schematic of the typical UPS system. In the present study, a multi chamber ultra high vacuum (UHV) surface science facility (VG Scientific/ RHK Technology) comprising of a $150 \mathrm{~mm}$ radius CLAM 4 hemispherical analyzer was used. A differentially pumped HE-discharge lamp was used for the UV radiation. 


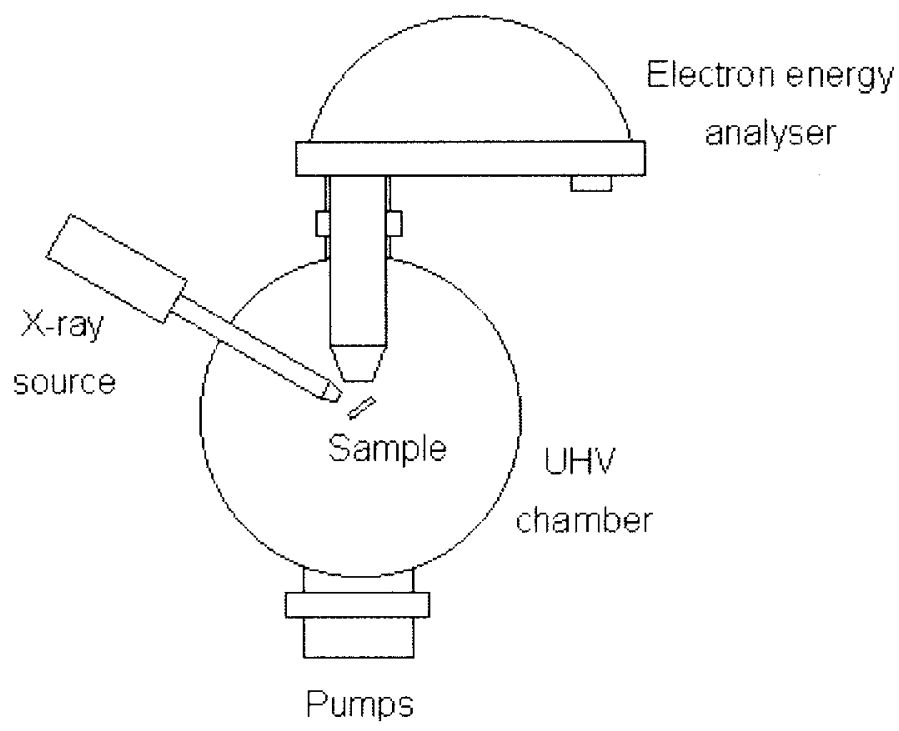

Figure 3.6 Schematic illustration of UPS chamber.

\subsubsection{Field electron emission characteristics}

Field emission measurements were performed on each sample in a custom build vacuum chamber operated at pressure of $10^{-7}$ Torr using a turbo pump. The chamber has ports to accommodate a variety of experimental devices, analytical instruments, viewports, feedthroughs and other accessories as necessary. The platinum wire with assynthesized CCNTs was placed in a V-groove of a copper plate which is further placed on a custom designed sample-holder. A flat copper anode was moved towards the CCNTs by means of a micro manipulator $(\sim 3.125 \mu \mathrm{m}$ step size $)$. A zero inter-electrode distance $(d=0)$ was established by observing a sudden change in resistance when the anode touches the CCNTs. Measurements were performed at 5 different distances $(d)$ by sweeping the voltage from $0-500 \mathrm{~V}$ while recording the current using a Keithley 6487 pico-ammeter equipped with a built in variable voltage source. The schematic of the set up is shown in the Figure 3.7. 

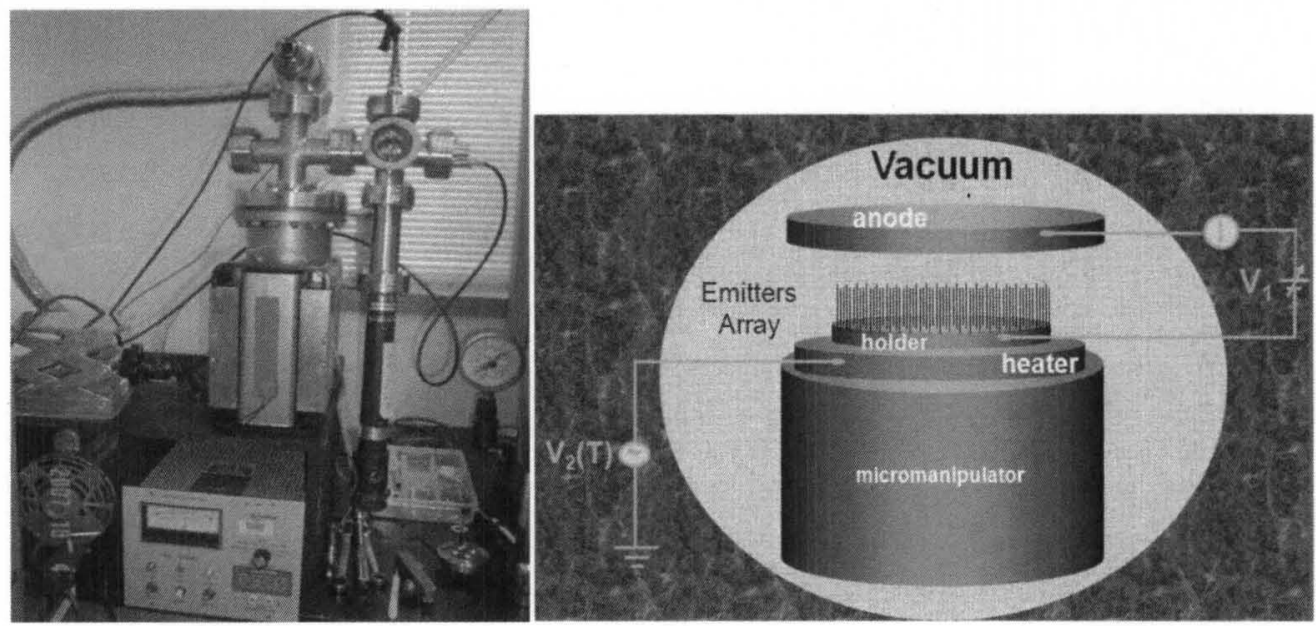

Figure 3.7 Field emission experimental set up showing (a) picture of the experimental chamber (b) schematic illustration of experimental set up inside the chamber.

\subsubsection{Thermionic emission characteristics}

Thermionic emission measurements were performed in a vacuum chamber at a pressure of $\sim 8 \times 10-7$ Torr. Two slightly different arrangements of sample stages have been employed as shown in the Figure 3.8. In the case of substrates consisting of platinum wire with as-synthesized CCNT's, the wire was placed in a V-groove of a copper plate as discussed above. A flat copper anode was moved towards the CCNTs by means of a micro manipulator (Figure 3.8a). In the case of conducting graphite foil with synthesized CCNT's (or CCNT's with diamond), the foil was placed on a ceramic plate (boron nitride). The plate was placed on the pyrolitic boron nitride (PBN) heater. The graphite foil was attached to the electrode, so that the sample acts as a cathode. A molybdenum anode was placed over the sample and was attached to the micromanipulator (Figure $3.8 \mathrm{~b})$. Zero distance $(\mathrm{d}=0)$ between cathode and anode was established by observing a sudden change in resistance when the anode touches the 
sample. Measurements were performed at set distance (d) for different temperatures by sweeping the voltage from 0 to $500 \mathrm{~V}$ while recording the current using a pico-ammeter (Keithley 6487) equipped with a built in variable voltage source. PBN heater was connected to separate power supply. Temperatures were measured using pyrometer.
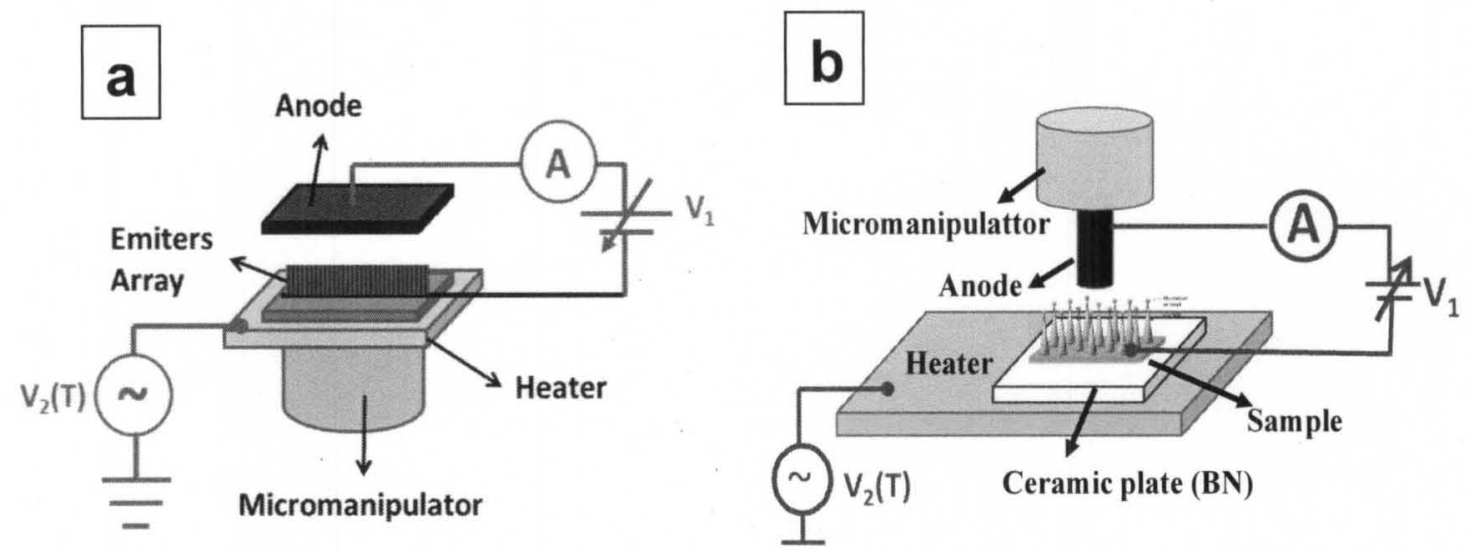

Figure 3.8 Schematic illustrations of thermionic emission experimental set-ups for (a) for CCNT's on platinum wire and (b) for CCNT's (or CCNT's with diamond) on graphite foil.

\subsection{Summary}

This chapter provided the description of various synthesis, material and field and thermionic electron emission characterization tools used in this work. Due to various nanostructures and different characteristic features and studies, a variety of characterization methods were employed to obtain complementary information of the structural and characteristic features of these nano structures. 


\section{CHAPTER 4}

\section{LARGE AREA SYNTHESIS OF CCNTS ON PLANAR SUBSTRATES}

\subsection{Introduction}

Conical carbon nanotubes (CCNTs) are morphological manifestation of MWCNT consisting of central MWCNT surrounded by helical graphitic sheets resulting into a conical geometry. As discussed in Chapter 2, there exist different conical carbon structures similar to CCNTs. However, CCNTs are interesting due to their unique features and have been shown to exhibit high sensitivity for neurotransmitter detection ${ }^{75}$ and superior field emission characteristics ${ }^{76}$.

These CCNTs, earlier called carbon nanopipettes (CNPs), were primarily synthesized on platinum or other wire substrates ${ }^{68}$. But, the practical applications of CCNTs were limited by thesmall area of the CCNTs and the wire geometry of the substrate. Though the synthesis of these structures on planar substrates was reported ${ }^{78,79}$, their reproducibility was not adequate. Moreover, all the different mechanisms suggested for evolution of conical morphology so far are not consistent with one another.

This chapter describes the synthesis of CCNTs in large area flat substrates and the study of the underlying nucleation mechanism for controlled synthesis. It also describes other nanostructures such as nanohorns and graphene superlattice formed at different experimental conditions. A similar experimental procedure described earlier in Chapter 3 
was employed along with the study of different catalysts and growth parameters. A proposed nucleation mechanism was verified, and a kinetic growth model was presented.

\subsection{Experimental procedures}

Several experiments were performed on graphite foils using different carbide forming catalytic metals (iron and nickel) to study the role of catalytic metal on the nucleation of CCNTs. Here, the catalyst metal wire ( $2 \mathrm{~cm}$ long) was supported by a hole drilled into the graphite rod around which the foil was rolled as shown in Figure 4.1.Upon the exposure to the plasma discharge, the catalyst metal evaporated onto the foil, which created an in-situ catalyst during the experiment. The melting of the catalyst metal into a spherical ball at the tip of graphite rod was clearly seen within few seconds of the plasma exposure. Similar experimental conditions such as 30-50 Torr pressure with microwave power of $900-1000 \mathrm{~W}$ using $1-1.5 \%$ methane in hydrogen mentioned earlier were employed. To study the effect of pressure along with different catalysts, experiments at higher pressures ranging from 60 to 75 Torr were also conducted.

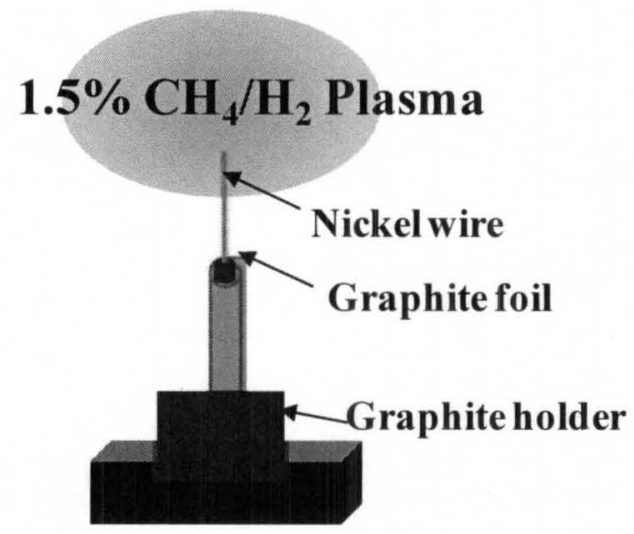

Figure 4.1 Schematic illustration of an experimental configuration for CCNTs with insitu nickel wire. 
Some more experiments were conducted on platinum wire substrates to verify the proposed nucleation mechanism. In these experiments, the CCNT arrays on wire substrate were post-treated by etching those to MWNTs by placing the substrate horizontally on the graphite susceptor. The experimental conditions include plasma power of $550 \mathrm{~W}$ and pressure of 25 Torr for approximately 30 minutes in hydrogen plasma. Later, the straight MWNT arrays were used for regrowth experiments by immersing the substrate vertically into the plasma operated at $980 \mathrm{~W}$ and 30 torr for 10 minutes with $1 \%$ methane in hydrogen $(200 \mathrm{sccm})$ using ASTeX 5010 reactor.

\subsection{Results}

\subsubsection{CCNTs with platinum catalyst}

The experiments using graphite foils sputtered with platinum resulted in CCNT arrays over an area as large as $0.5-1 \mathrm{~cm}^{2}$ as shown in figure $4.2 \mathrm{a}$. The resulting CCNTs were as long as 30 to $40 \mu \mathrm{m}$ from 40 -minute synthesis. The growth rates were estimated at approximately 60 microns per hour, which is an order of magnitude higher compared to results on wire substrates. The density of the resulting CCNTs is $10^{6} / \mathrm{cm}^{2}$. High aspect ratio of a typical CCNT is shown in Figure $4.2 \mathrm{~b}$ with inset showing the close-up view of the open tip. CCNTs exhibit a smooth surface as shown in the Figure $4.2 \mathrm{~b}$, which consists of concentric cylindrical graphene sheets (epitaxial growth) around the central MWNT as shown in Figure 4.2c. In some cases, after attaining conical shape, some structures have exhibited interesting growth, i.e., spiral coiling of multi-layer graphite sheets around the original CCNT as shown in Figure 4.2d. The inset clearly shows the spiraling effect of multilayer graphene sheets around CCNT. The resulting CCNTs on the foil substrates taper from the lowest base level into perfect conical structures, even up to lengths of 75 
microns compared to those obtained on wire substrates. Results also suggested that the deposition area can be increased by increasing either the diameter or the height of the foil immersed into the plasma. The upper limit on the diameter of the rolled foil depends upon the size of the plasma discharge (1 inch in diameter for the reactor used in the present studies). The density of CCNTs on tungsten metal foils is 1 to 2 orders of magnitude lower than on graphite foil with significant variation in their uniformity. Moreover, after the synthesis, the tungsten foil became very brittle making it difficult to handle. Thus, for fundamental studies geared towards the understanding of such properties, graphite foils are chosen as appropriate.
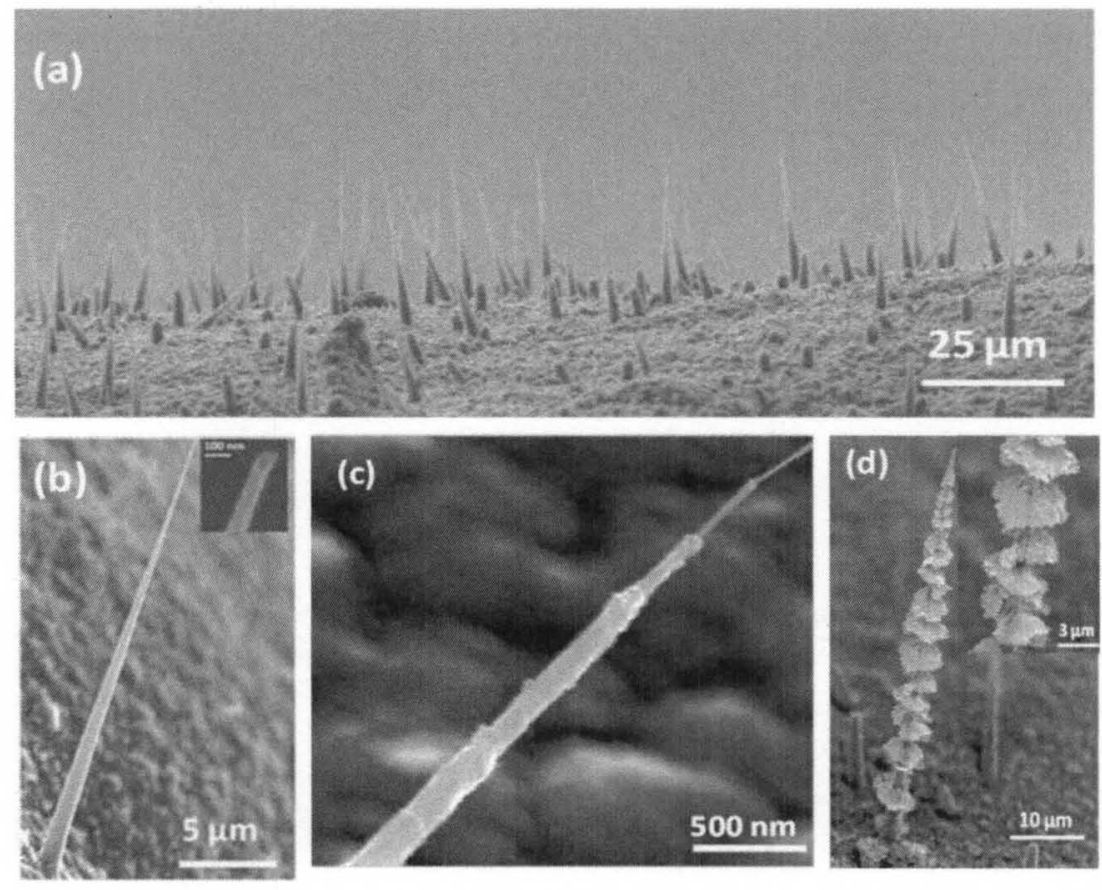

Figure 4.2 SEM images showing (a) an array of CCNTs on graphite foil, (b) an individual CCNT with inset showing the open-ended tip and (c) surface of CCNT consisting of concentric cylindrical graphene sheets and (d) non epitaxial coiling of graphene sheets around the CCNT. 


\subsubsection{CCNTs with iron and nickel catalyst}

The second set of experiments using nickel and iron as catalytic metals resulted in different morphological variations in conical structures as shown in Figure 4.3a. Some of them had sharp tips (similar to Figure 4.2b), some blunt tips (Figure 4.3b), and others developed an inverted conical shape starting from the sharp tip and ending with a big bulb (Figure 4.3c). The resulting density of CCNTs in all the experiments was on the order of $10^{6} / \mathrm{cm}^{2}$ irrespective of the type of catalyst metal or the deposition method employed on graphite foils.
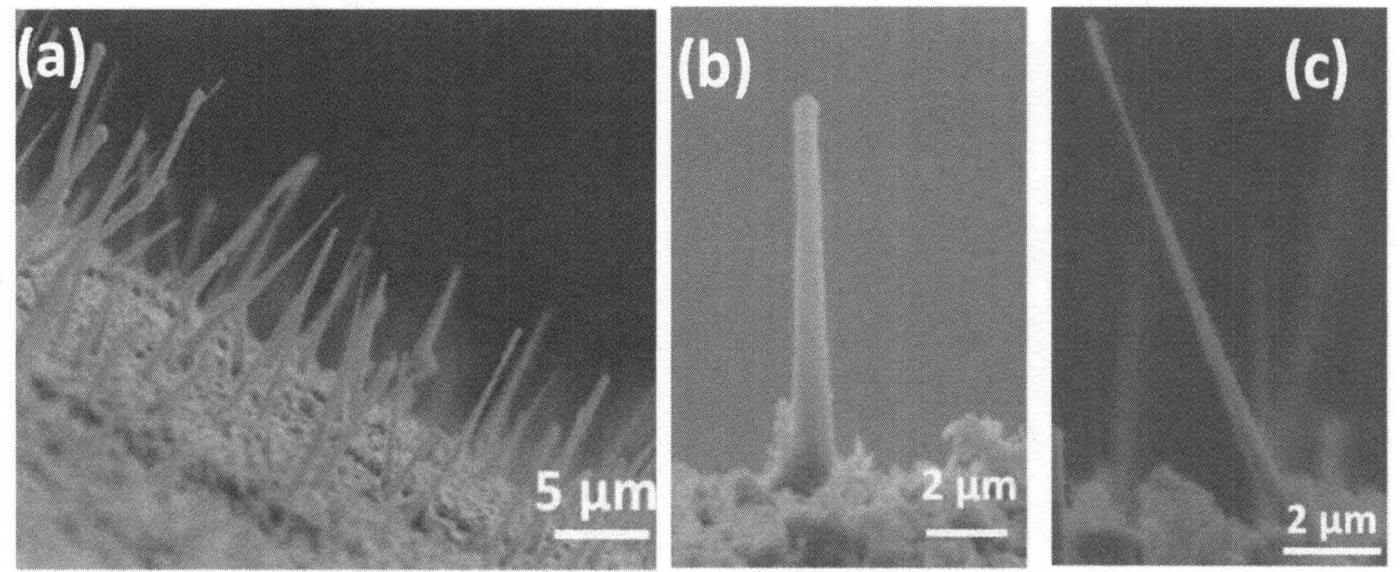

Figure 4.3 SEM images of (a) an array of CCNTs with different morphologies resulted during experiments with nickel wire, (b) an individual CCNT with a blunt tip and (c) an individual CCNT with inverted conical tip.

Further analysis of these structures was carried out using transmission electron microscopy (TEM). Figure 4.4a, suggests that typical CCNT with sharp tips maintain central hollow core with no catalyst at the tips. The closing of tip can be attributed to the presence of a hydrogen-dominant plasma environment favoring the curving of the 
graphene sheets ${ }^{96}$. The bulb-like tip of the conical structure shown in Figure $4.4 \mathrm{~b}$ was examined extensively using transmission electron microscope and was found to be devoid of any metal. The presence of existing inner hollow core can be seen from Figure 4.4c. The high-resolution image of bulb-like tip shown in the inset of Figure $4.4 \mathrm{~d}$ clearly displays the presence of graphitic planes rolling around in a circular fashion forming a spherical tip, not the catalyst. In the case of the CCNT with a morphology similar to Figure $4.3 \mathrm{c}$, the inner hollow core extends to the end, kinks at the tip (as shown in 4.4e), and extends expansive growth to form the inverted conical geometry. Around this region, the hollow core becomes closed and does seem to continue the other side with limited allowance. However, such spherical graphitic and related variations at tips were only observed in fraction of the resulting structures and also only in experiments that used iron and nickel as catalysts. These observations indicate that the spherical graphitic clusters do not play any significant role in the formation of CCNTs. 

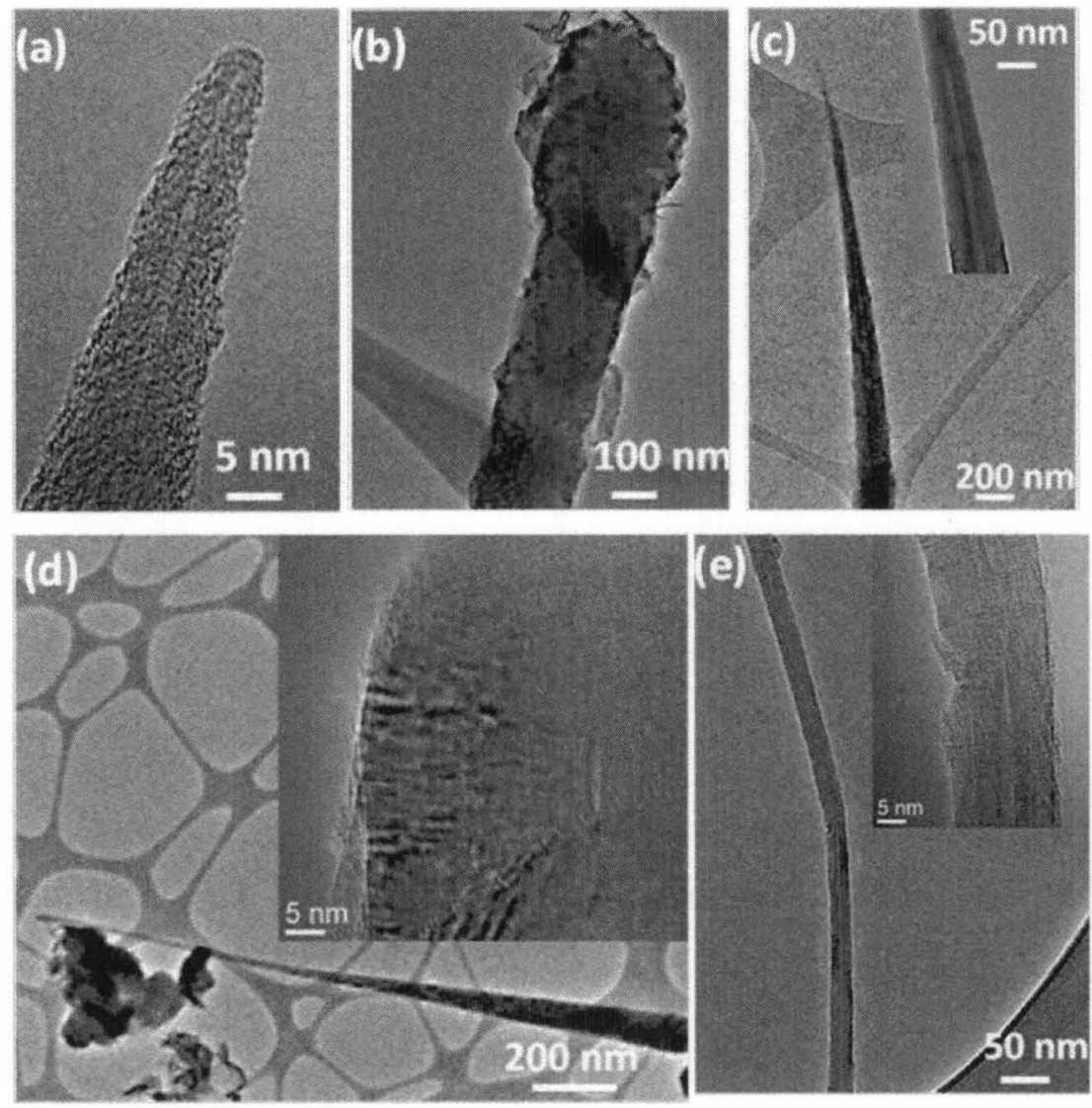

Figure 4.4 TEM images of CCNTs showing the absence of catalyst (a) CCNTs with sharp tip, (b) CCNTs with blunt tip, (c) CCNTs with inset showing the presence of a hollow core, (d) CCNTs with inverted conical tip with inset showing the high resolution image of the tip consisting of graphitic planes and (e) high-resolution image of a kink at the tip with inset showing the abrupt change in the hollow core.

\subsubsection{Role of the catalyst and graphite rod}

CCNTs were also observed in experiments without using any catalyst metal, but the densities were 2-3 orders of magnitude lower, which suggest an important role of the catalyst metal in the nucleation of CCNTs. The observed nucleation can be due to the presence of residual catalyst contamination in the reactor from earlier experiments. Based 
on some of SEM observations, smaller catalyst metal clusters in the initial stages seem to either evaporate quickly from graphite foil substrates or agglomerate into larger clusters that result in low density CCNTs. It was also observed that the lower the thickness of the catalyst film, the smaller the diameter of the catalyst droplets formed. Therefore, the substrate pretreatment and the catalyst optimization seem necessary to increase the density of CCNTs. In the given pressure range of 30-50 torr, the temperature at the center of plasma (near the tip of foil substrate) could reach as high as $1800^{\circ} \mathrm{C}$, higher than the melting point of the platinum observed in our present and earlier report ${ }^{68}$.

Experiments with no graphite rod inside the foil showed no difference in the resulting density of CCNTs, but excess deposition of carbon was observed on the grown CCNTs. The following SEM images (Figure 4.5) clearly show the extra deposition of carbon on the CCNTs resulting in the loss of its perfect tapering geometry. It was also observed that this extra carbon deposition on CCNTs was seen specifically at the very tip of the foil where the excess build up of heat could take place mainly in the absence of the rod. This could result in the observed extra deposition of carbon especially during the sudden shut down of the hydrocarbon supply. Thus the presence of rod ensure the proper conduction of this heat across the foil preventing the excess build up of heat. This could be the reason for the observed uniformity in the CCNTs without any excess carbon deposition in the presence of rod. Similar change in the morphology with extra carbon deposition was also observed at higher pressures (60 torr), indicating the role of higher temperatures in the observed variation. 

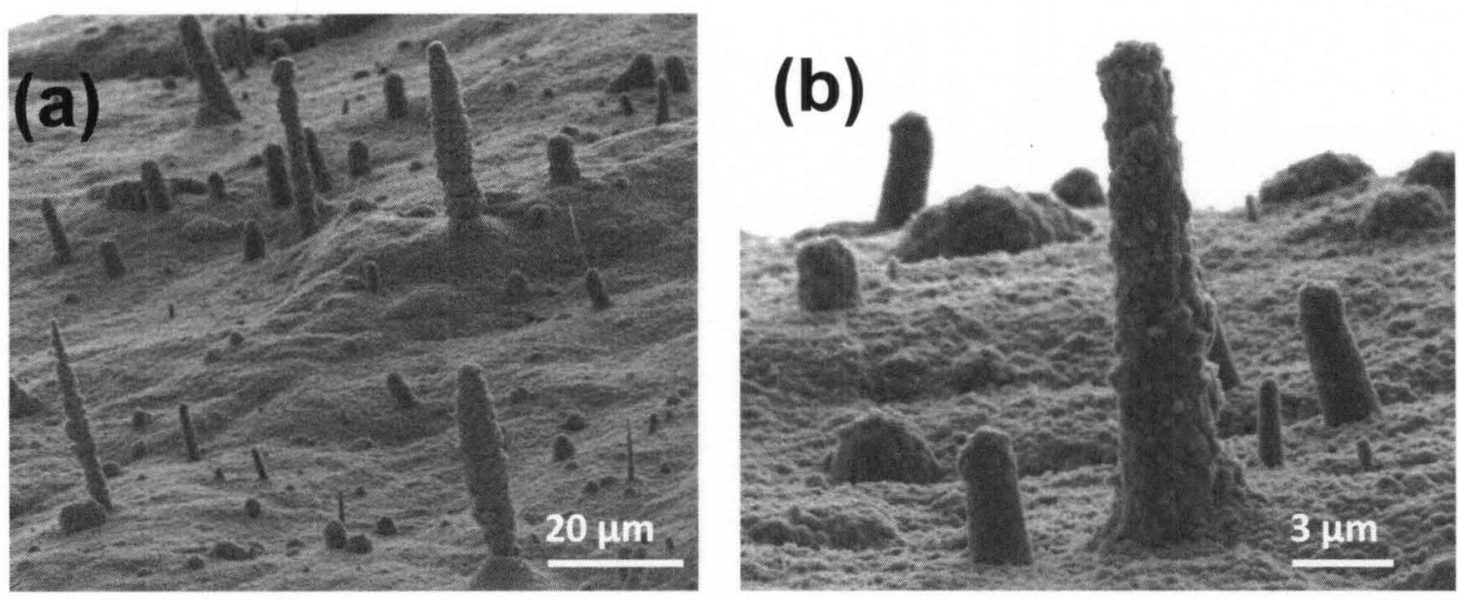

Figure 4.5 SEM images of (a) array of CCNTs (b) close up image of the individual CCNT with extra carbon deposition on the surface.

\subsubsection{Carbon nanohorns}

Experiments conducted at higher pressures of 75 torr using in-situ nickel catalyst for about 5 minutes resulted in a black deposition on graphite foil. TEM studies revealed the formation of onions, rings, polyhedral shells, and nanohorns. Most of them consisted of tubular structures with conical tips as shown in Figure 4.6a, with individual multiwalled carbon nanohorns shown in Figure 4.6b. Similar observations were reported during the arc discharge process ${ }^{74,97}$. Although the synthesis of carbon nanohorns was attributed either to the presence and modification in the catalyst particle in few studies $^{98,99}$ or to reaction of catalyst powders ${ }^{100}$, the structures were one order of magnitude larger than the present ones. Experiments performed with different and no catalyst also resulted in similar findings, suggesting the role of higher synthesis temperatures on the nucleation of graphene layers but not the catalyst. Harsh conditions employed in the earlier studies ${ }^{74,97}$ have also resulted in similar structures without the use of any catalyst. In microwave plasma chemical vapor deposition reactor, higher pressures 
lead to higher temperatures on substrates immersed in plasma discharges. So, our experiments at higher pressures in microwave plasmas suggest even harsher environment (temperatures in excess of $2500{ }^{\circ} \mathrm{C}$ ) leading to spontaneous nucleation and growth of carbon onions, rings and conical carbon tube morphologies without the use of metal catalysts ${ }^{74}$.
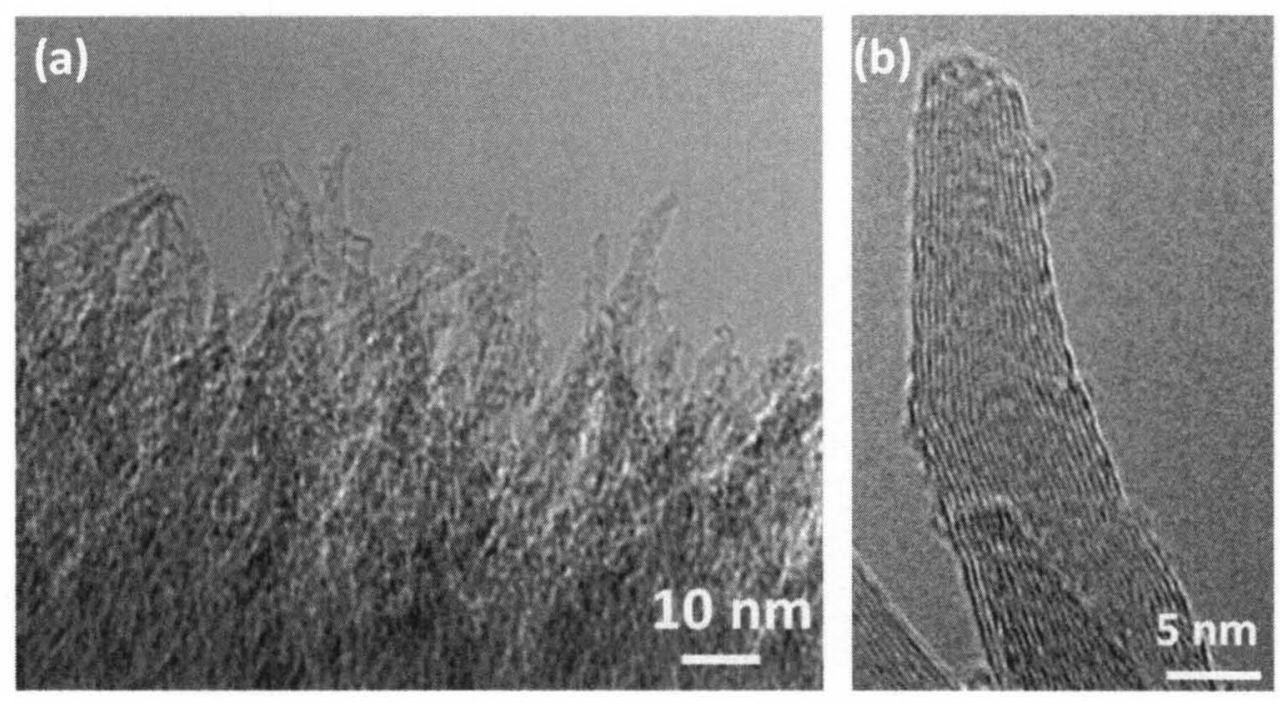

Figure 4.6 TEM images of (a) bundle of nanohorns and (b) a high-resolution image of individual multi-walled nanohorn.

\subsection{Nucleation and growth mechanism}

Based on all the above results with CCNTs, it can be inferred that nucleation is initiated with a MWNT from small catalyst clusters that have critical dimensions. The underlying mechanism is similar to tip-led growth described earlier for MWNT ${ }^{101,102}$. Therefore, any agglomeration of catalyst clusters in the initial stages could reduce the resulting density of CCNTs. If the growth was attributed to a shrinking catalyst mechanism ${ }^{64}$, the presence of catalyst at the tip should be evidently seen at least in short duration experiments. However, an open-ended tip with absence of catalyst was clearly 
shown in Figure 4.4a. Short term experiments $(<5 \mathrm{~min})$ were also conducted to observe if there is any presence of the catalyst in the early stages of the growth. The resulted CCNTs had smaller base diameters and lengths less than 1 micron, which also did not show the presence of any catalyst particles at their tips or bases clearly evident from the TEM image and elemental analysis as shown in Figure 4.7. This further validates that radial growth continues without the assistance of any catalyst.

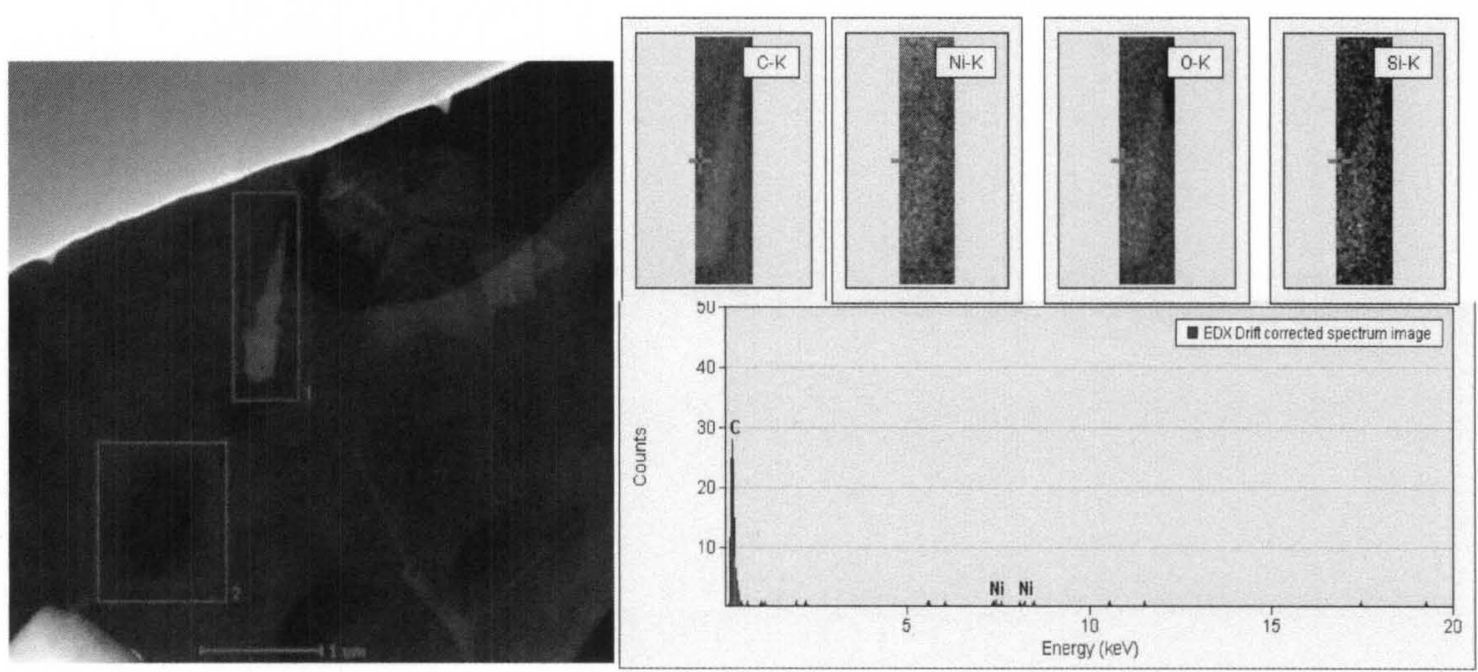

Figure 4.7 TEM image of CCNT with its elemental analysis revealing the absence of any metal catalyst at either tip or base.

It was previously speculated that a large catalyst cluster at the base of growing conical structure assisting the initiation of new layers for radial growth at the base ${ }^{71,78}$. As such, to examine the interface between the substrate and the base of CCNTs, tungsten foils were chosen instead of graphite for ease of handling and clear observation of interface. The cross-sectional SEM images in Figures 4.8a and 4.8b show that the conical structure grew directly from the tungsten foil and does not have any big catalyst particle at the base. In addition, though a continuous supply of the catalyst was claimed to be 
necessary for the growth of tubular graphite cones ${ }^{78}$, it was achieved in case of limited catalyst $^{79}$, with the actual role of catalyst not apparent. However our findings suggest that the formation of new graphene layers at the base could occur without the help of a catalyst, which is further verified by regrowth experiments discussed below.
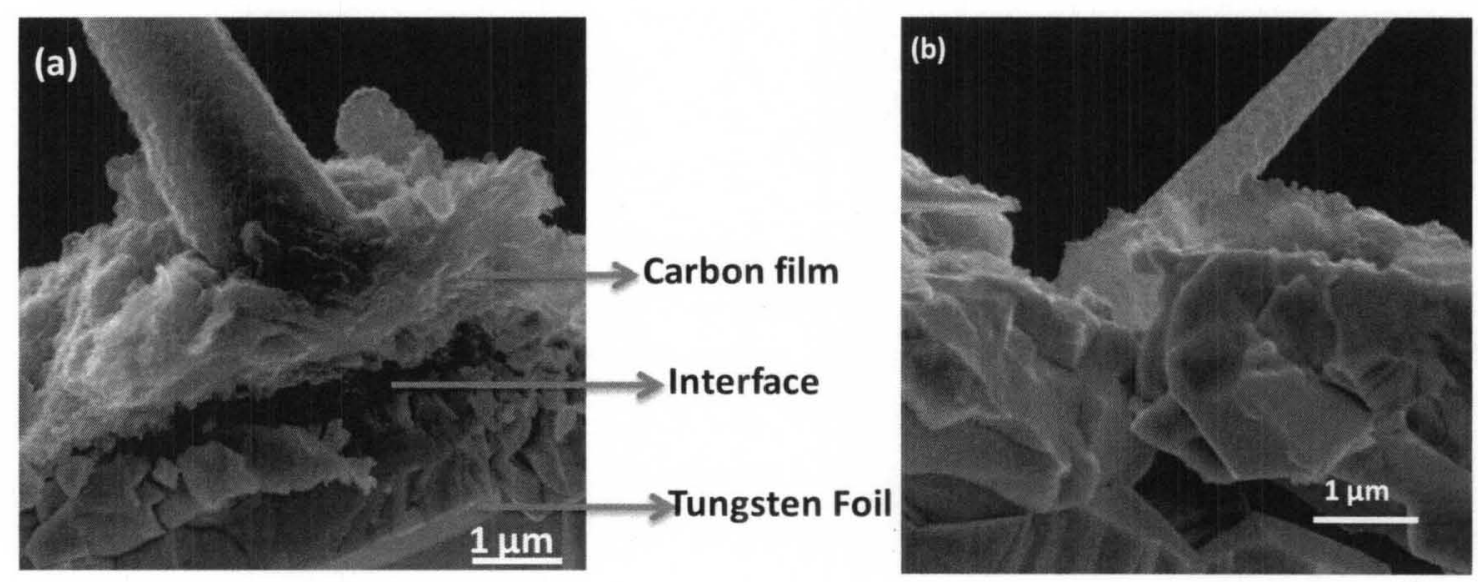

Figure 4.8 (a) SEM image showing the side, cross-sectional view of a CCNT grown on tungsten foil substrate and (b) SEM image of a broken CCNT with inset showing the absence of a catalyst particle at the base.

All the above experimental observations suggest a simpler mechanism for nucleation and growth for CCNTs, which is shown schematically in Figure 4.9. In the initial stages, first the formation of catalyst metal clusters on the substrate takes place (Figure 4.9a), then straight MWNTs nucleate with the help of small catalyst metal clusters at their tips (Figure 4.9b). Later, the MWNT continue to grow vertically, but at the same time, the nucleation of new graphene layers occurs at the bass as shown in Figure $4.9 \mathrm{c}$. In the fourth step, the small metal catalyst clusters will be evaporated along with continuous growth of new epitaxial layers (Figure 4.9d). The epitaxial growth of such graphene sheets in the form of concentric cylinders around the central MWNT can 
be evidently seen in Figure 4.2c. Finally, the conical shape evolves with a steady state vertical growth by the addition of carbon adatoms at exposed edge planes across its surface and radial growth through the addition of new graphene layers with no catalyst at the tip or base (Figure 4.9e). In few cases, once the CCNT attains the conical shape, additional spiral coiling of multi-layer graphite sheets around the original CCNT could be taking place as mentioned earlier (Figure 4.2d). The presence of elevated temperatures $\left(>1800{ }^{\circ} \mathrm{C}\right)$ higher than the melting point of the platinum (observed in our present and earlier report ${ }^{68}$ ) and strong electric field discharge at the tip of coiled foil immersed in the center of the plasma, force the evaporation of catalyst clusters at the tips. This is because the radical recombination rates can be severe on the catalytic metal clusters and result in heating. The temperature of the catalyst cluster supported at the tip being heated continuously can increase rapidly due to poor thermal transport back to substrate. A simple thermal transport analysis was earlier presented for diamond crystals at tips of $\mathrm{CCNTs}^{96}$. In our case, higher recombination rates due to high densities of radicals within the plasma discharge can easily heat and evaporate the catalyst particle. Similarly, the presence of high energy ions and various radical species from the electron impacts and neutral reactions has been shown to influence plasma assisted CVD ${ }^{102,103}$. In the case of thermal CVD, only surface reactions play a dominant role ${ }^{103}$. Other studies have also observed the evaporation of the catalyst with thermal annealing at high temperatures $\left(>2000^{\circ} \mathrm{C}\right)$ in the case of carbon nanofibers ${ }^{104}$. 
(a)

(b)

(c) Catalyst assisted growth of MWNT

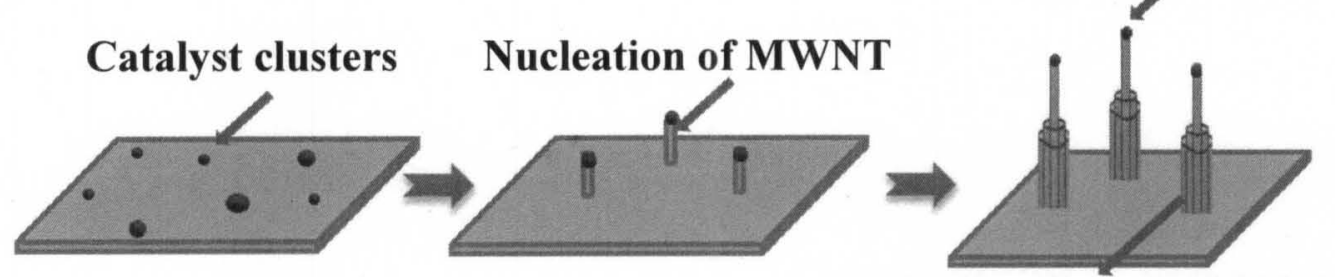

Nucleation of new graphitic layers

(e)

(d)
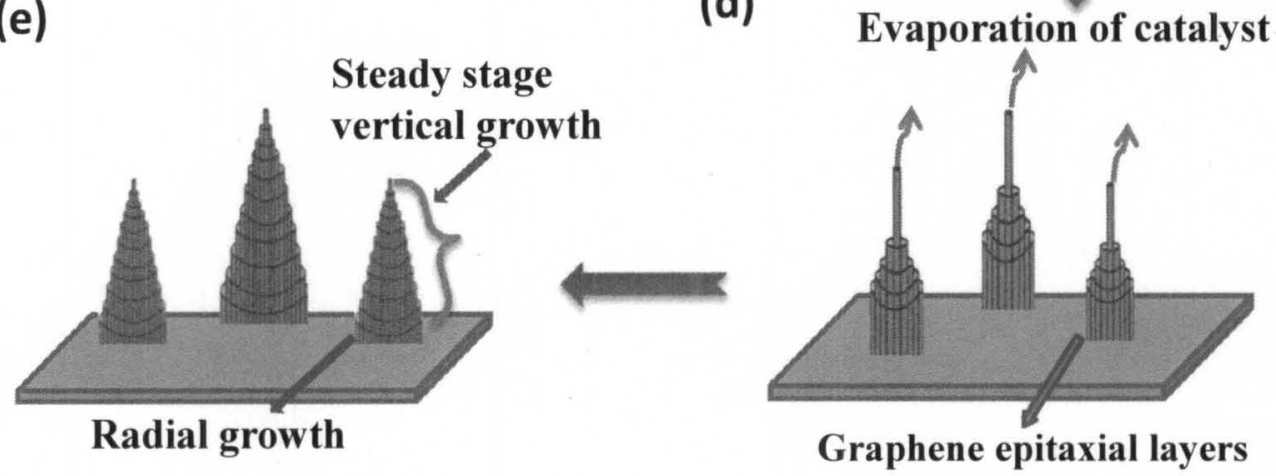

Figure 4.9 A schematic illustration of the suggested nucleation and growth mechanism for CCNTs (a) formation of small catalyst clusters on the substrate, (b) nucleation of a MWNT, (c) continued catalyst-assisted growth of MWNT along with the nucleation of new graphene layers, (d) evaporation of the catalyst with continued growth of epitaxial layers and (e) continued radial growth via addition of graphene layers and steady state vertical growth via addition of carbon atoms.

\subsubsection{Regrowth experiments}

Proposed mechanism is confirmed from the regrowth experiments discussed in the experimental section. CCNT arrays were etched to MWNTs using hydrogen plasma (Figure 4.10a) followed by regrowth in methane and hydrogen plasma. Figure 4.10b shows the growth of conical structures in the same region with the inset showing the 
individual CCNT grown from the MWNT. The regrowth experiments resulted in the deposition of a thick carbon layer, which undermines the actual length of regrown conical structures compared to their original lengths. The results indicate that the evolution of conical morphology takes place through the nucleation and growth of new graphene layers in the presence of higher synthesis temperatures which was also observed in the case of graphite poly crystals ${ }^{105}$ and graphite whiskers ${ }^{106}$. In some cases, heat treatment through high temperatures $\left(1500-2800{ }^{\circ} \mathrm{C}\right)$ has been attributed to the observed purely crystalline graphitic character of carbon nanofibers ${ }^{104}$. Similarly the growth of crystalline graphitic sheets in the present case of CCNTs can also be attributed to the existence of such high temperatures, which helps in perfect crystalline graphitization preventing the formation of any amorphous carbon ${ }^{107,104,106}$. The presence and dominance of higher molecular species of carbon resulting from the plasma CVD can also help with the epitaxial nucleation of new graphitic sheets ${ }^{105}$.
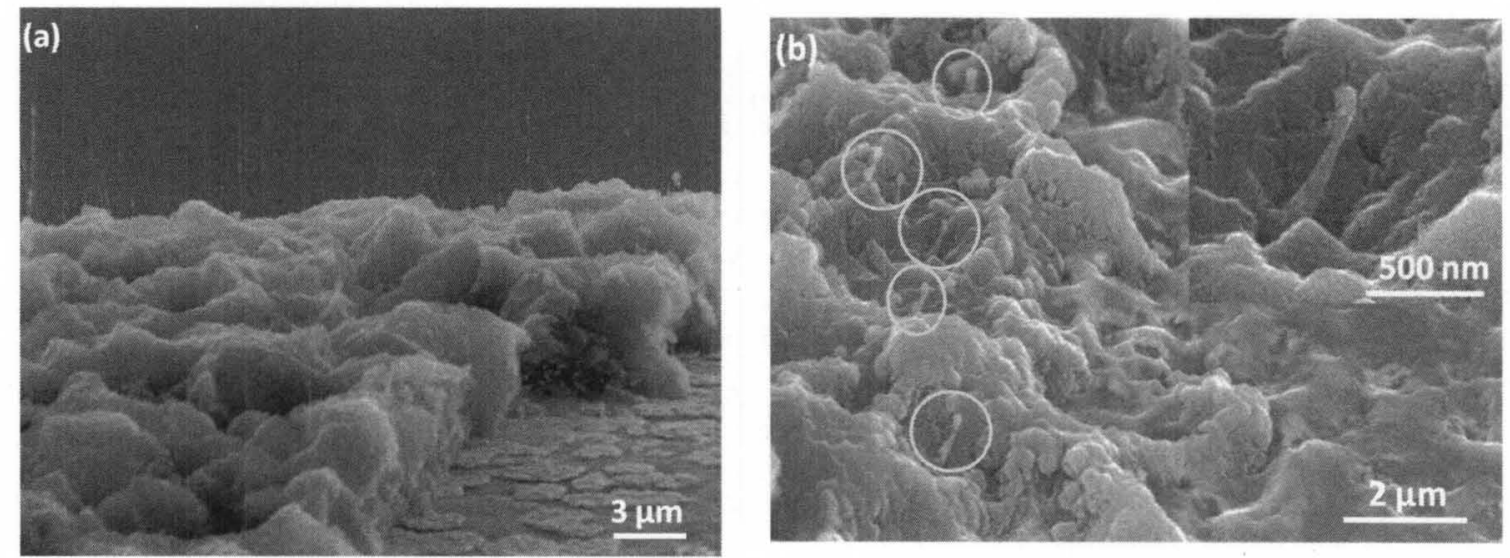

Figure 4.10 SEM image of (a) CCNTs etched to straight MWNTs and (b) regrowth of etched CCNT to conical structures with inset showing magnified view of one of the CCNTs circled. 


\subsubsection{Kinetic growth model}

The above growth model could be further understood by examining the growth kinetics of CCNTs. At the given experimental conditions (several tens of torr and 1 - to 2 $\%$ methane in hydrogen), the mean free path lengths of growth species (hydrocarbon and atomic hydrogen radicals) are estimated to be on the order of several tens of microns. Under such conditions, the flux reaching the growing nanostructure in the initial stages will be same as that for a flat surface. At sizes below hundreds of microns, the growth of CCNTs can only be limited by impingement kinetics. In this case, the volumetric growth rate will be directly proportional to impingement rate. The number of molecules impinging per unit time (I) can be derived as:

$$
\mathrm{I}(\# / \mathrm{s})=\mathrm{F} \times\left(\pi d^{2} / 4\right)
$$

Where, $\mathrm{F}$ is the molecular impingement flux $\left(\# / \mathrm{s} * \mathrm{~m}^{2}\right)$ and $\pi d^{2}$ is the cylindrical crosssectional area $\left(\mathrm{m}^{2}\right)$ with $d$ being the base diameter of the conical structure. Volumetric growth rate, $\mathrm{V}$ (volume increase per unit time) can be derived as:

$$
\mathrm{V}\left(\mathrm{m}^{3} / \mathrm{s}\right)=\text { Linear growth rate }(\mathrm{m} / \mathrm{s}) \times \pi \frac{d}{2} \sqrt{l^{2}+\left(\frac{d}{2}\right)^{2}}
$$

Where, $\left(\pi \frac{d}{2} \sqrt{l^{2}+\left(\frac{d}{2}\right)^{2}}\right.$ is the surface area $\left(\mathrm{m}^{2}\right)$ of the cone with $l$ and $d$ being the length and diameter of the cone. The number of molecules forming one molar volume per unit time $(\mathrm{M})$ can be given by

$$
\mathrm{M}(\# / \mathrm{s})=\mathrm{V} \times \Omega
$$


Where $\Omega$ is the number of molecule attaching per unit volume $\left(\# / \mathrm{m}^{3}\right)$. Equating the number of molecules resulting from impinging flux (I) to the number of molecules forming a molar volume (M) and assuming $F$ and $\Omega$ as constants, the growth rate can be derived as:

$$
\text { Linear growth rate } \propto\left(d /\left(\sqrt{l^{2}+\left(\frac{d}{2}\right)^{2}}\right)\right)
$$

With the diameter of the conical structure being one order of magnitude smaller than the length $(d<<l)$, the growth rate can be finally simplified as:

$$
\text { Linear growth rate } \propto(d / l)
$$

In the case of cylindrical structures, the growth rate should be constant irrespective of the length and diameter under the attachment kinetics limitations.

To verify the kinetic impingement limited growth for CCNTs, we conducted a series of experiments on graphite foils using the similar conditions mentioned in the experimental section 2.1 at different time scales. The average length $(l)$ and diameter $(d)$ of CCNT at each time scale was determined from SEM images. The instantaneous growth rates were calculated from the slopes at different time scales for the data shown in Figure 4.11a. The instantaneous growth rates were plotted against the $(d / l)$ as shown in Figure $4.11 \mathrm{~b}$, which shows that the growth rate is linearly proportional to the ratio of diameter to length $(d / l)$ of the CCNT. Additional investigations would be needed to extend the range of the $d / l$ values for further understanding of relationship between the growth rate and individual parameter. 

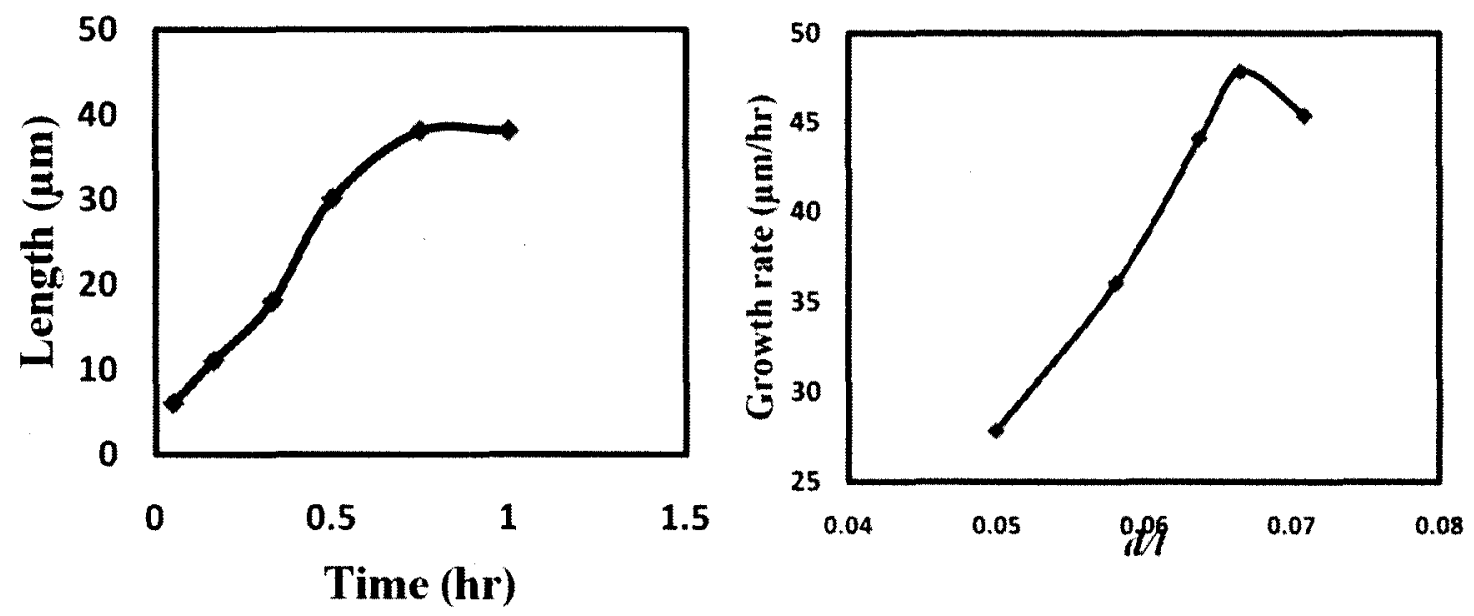

Figure 4.11 (a) Plot showing the length of conical structures at various time scales during the growth and (b) plot showing linear relationship between the growth rate and the ratio of the diameter to length $(d / l)$ of the conical structures

\subsection{Graphene superlattice}

Experiments at higher pressures also resulted in observation of some special Moire patterns of formed few layer graphene samples along with nanohorns. There is almost unanimous agreement that the triangular superlattice is the Moire pattern produced by rotational misorientation between the neighbouring graphite layers. Moire patterns were first studied by scanning tunneling microscopy (STM) for several decades. Recently, Transmission electron microscope (TEM) has also been successfully used to study the triangular superlattices found in few layer graphene (FLG) sampes prepared by various methods ${ }^{108,109,110}$. However, TEM observation of adjacent Moire patterns consisting of triangular superlattice and parallel straight bands in FLG samples prepared by CVD has not been known. 
Figure 4.12a shows a TEM micrograph of a $250 \mathrm{~nm} \times 350 \mathrm{~nm}$ section of FLG. In this figure, the FLG consists of several layers, some of very small areas. A region of FLG shows a superlattice of triangular symmetry (highlighted outlines) which appears similar, in structure as well as in definition, to STM images of bulk graphite superlattice. The periodicity, D, of the superlattice obtained from this image (Fig. $4.12 \mathrm{a}$ ) is $\sim 5.8 \mathrm{~nm}$. Fast Fourier transform (FFT) of the superlattice region in Fig. 4.12a is indexed using the [0001] zone axis of graphite as shown in Figure 4.12b. A small radial splitting of $\sim 1.3^{\circ}$ of all (10-10)-type spots can be seen in this FFT with magnified region shown in the inset. This indicates the presence of two overlapping graphene layers with a relative rotation of $\sim 1.3^{\circ}$. Substitution of the values of $\mathrm{D}$ and $\theta$ in the equation for the rotational Moire pattern: $\mathrm{D}=\mathrm{d} /[2 \sin \theta / 2]$ yields a value $\sim 1.3 \mathrm{~A}^{\circ}$ for lattice $\mathrm{d}$-spacing. This value agrees, within the experimental error, with $1.23 \mathrm{~A}^{\circ}$ which is the $\mathrm{d}$-spacing of the $(-12-10)$ planes. This is also consistent with the orientation of the pattern as the Moire fringes should be normal to the g-vector difference. Along with the triangular superlattice, Figure 4.11a shows an adjoining region of one-dimensional linear bands which originate from the bottom edge of the triangular superlattice (highlighted outlines). The 1-d pattern contains the same number of intensity maxima and minima as the terminating 2-d lattice and the two distinct regions of 2-d and 1-d intensity variations are joined to each other smoothly without any breakage of bonds. The number of bands is equal to the number of terminating superlattice maxima ${ }^{111,112}$. 

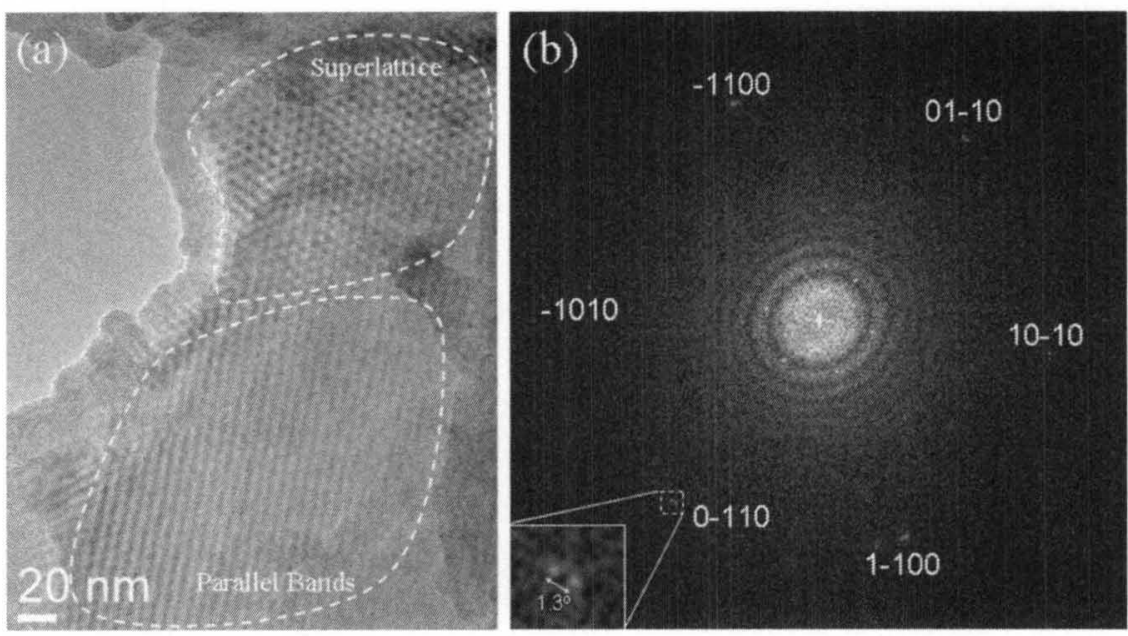

Figure 4.12 (a) TEM micrograph (250 nm x $350 \mathrm{~nm}$ ) of FLG graphene prepared by CVD. A superlattice is seen in the top section and parallel bands are seen in the bottom part (highlighted outlines), (b) FFT of HRTEM image indexed using [0001] graphite zone axis. Notice the radial splitting of $1.3^{\circ}$ present for all (10-10)-type spots. An enlarged view of the (0-110) spot splitting is shown in the inset

The observed unique Moire pattern consisting of superlatice and linear bands was answered by modeling of the adjacent Moire patterns by producing appropriate deformations of a layer of a two layer rectangular graphene. A pictorial modeling of two graphite layers is attempted in Figure 4.13 to prove that the supperlattice region and the adjacent region of linear bands are Moire patterns produced by placing a layer consisting of a rotated region and a parallelogram shaped region above a normal graphene layer. In Figure 4.13 , carbon atoms of the bottom layer are blue in color and the top layer atoms are red. As the top section of the graphene sheet is rotated, a Moire pattern of triangular superlattice becomes visible. Note that for clarity, the angle of rotation is selected as $5^{\circ}$ rather than the experimentally obtained rotation angle of $1.3^{\circ}$. The bottom part of Figure 
4.13 shows the Moire pattern obtained by super-position of a graphene layer of parallelogram shape, with right corner angle of $85^{\circ}$, over a rectangular graphene layer. In this bottom part, three distinct types of bands are seen: (1) bands of aa structure, (2) bands with aa structure, and (3) bands of aba structure. The bands with aa structure in the middle have maximum intensity. The bands with aba structure have brightness less than that of aa bands. For aa bands, the intensity should be close to that of perfect aa stacking. Therefore, the number of bright bands is equal to the number of terminating superlattice maxima and bands originate from superlattice maxima. The Moire patterns of the two sections, rotated top section and parallelogram shaped section in the bottom, reproduce the experimental results of superlattice and parallel bands. However, within experimental limitations, no difference in intensity variation between regions of aa and aa stacking is seen in Figure 4.12a. In Figure 4.13, the angle between a line drawn through the centers of the maxima in the triangular superlattice and a line along the middle of a linear band is $5^{\circ}$ which is equal to the angle of rotation of the superlattice region. The angle between similarly drawn lines in Figure $4.12 \mathrm{a}$ is $1.3^{\circ}$, equal to the calculated angle of rotation obtained from FFT. 

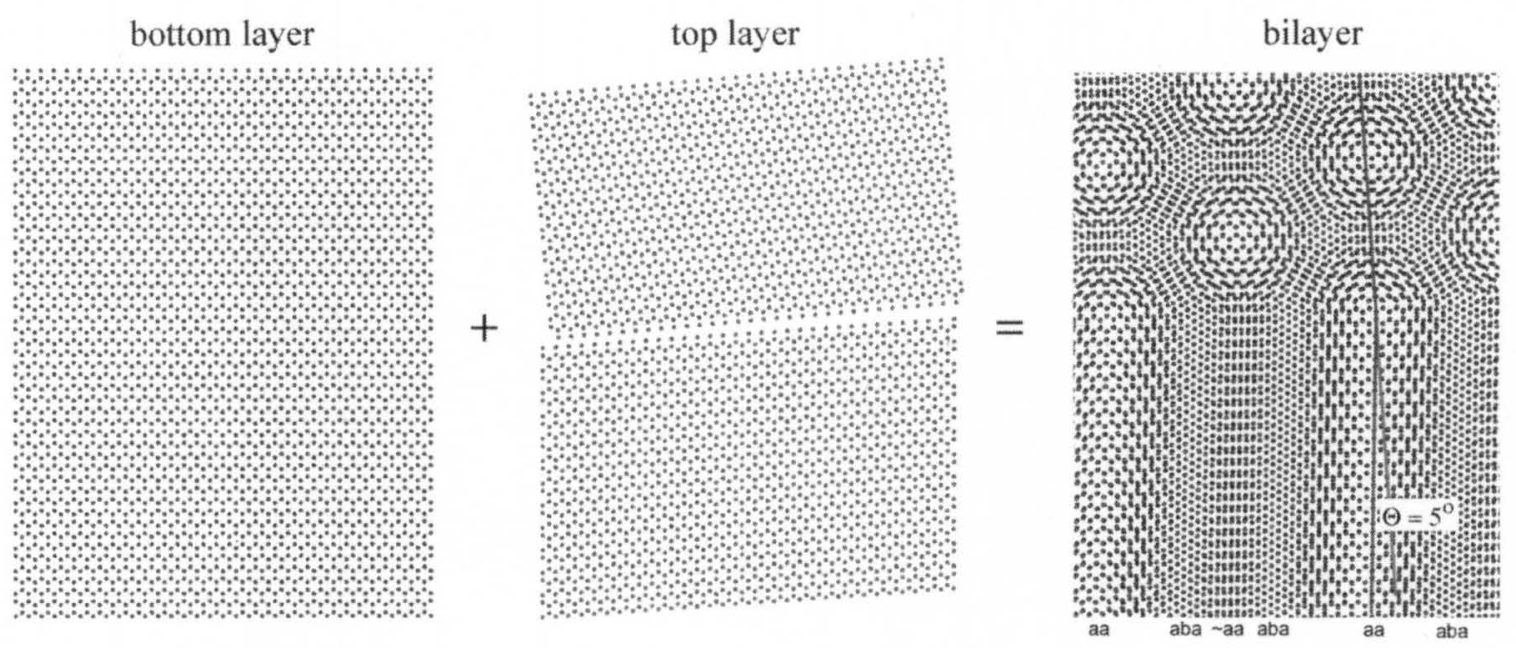

Figure 4.13 Model of two layer graphene sheets (blue bottom layer and red top layer).

The top part of the top (red) layer is rotated through $5^{\circ}$ and the shape of the bottom part is changed to a parallelogram with an $85^{\circ}$ top right hand corner. The two sections are continuous without any break in the chemical bonds. Regions of aa, aba, and aa are indicated below the corresponding regions. Schematics of each layer are shown for clarity.

\subsection{Summary}

In this chapter a method of preparing arrays of CCNTs over a large area on flat graphite and tungsten foil substrates is presented. The aspect ratio and the uniformity of the structures obtained were superior to those using wire substrates. However, it was found that the density of resulting CCNT arrays was limited to $10^{6} / \mathrm{cm} 2$ and further optimization of catalyst particle size distribution in the initial stages might be necessary to increase the nucleation density. It was also shown that higher synthesis pressures resulted in nanohorns, rings, polyhedral shells and super lattice structures. The growth 
mechanism was proposed which was also verified by regrowth experiments on MWNTs. From the growth kinetics data, it was shown that the growth rate is linearly proportional to the ratio of diameter to length $(d / t)$ of the $\mathrm{CCNT}$, which validates the kinetic impingement limited growth model for CCNTs. A pictorial model for the observed Moire pattern for the few layer graphene superlattice was also presented. 


\title{
CHAPTER 5
}

\section{FIELD AND THERMIONIC EMISSION CHARACTERISTICS OF CCNT}

\begin{abstract}
ARRAYS
\end{abstract}

\subsection{Introduction}

Field emission from CNTs has been explored due to their good mechanical, electrical, thermal and chemical properties along with high aspect ratios. In addition, the dependencies of field emission properties on various characteristic features of CNTs have also been reported ${ }^{61}$. However, as mentioned earlier in chapter 2, CCNTs exhibit the stability of a conical geometry combined with the advantage of cylindrical morphology for field and thermionic emission at lower electric fields. These conical structures with superior characteristic features including higher aspect ratios are expected to be mechanically and thermally more stable and can sustain greater current densities compared to constant diameter narrow cylinders i.e. CNTs. Though few reports on field emission characteristics of tapered morphologies have been reported ${ }^{113}$, the extent of these studies were limited and the structures do not represent the ideal conical morphology of CCNTs. Similarly though thermionic emission from pristine carbon nanotubes was recently studied ${ }^{27.73}$. these ideal conical structures have not been explored. This chapter describes controlled synthesis of several CCNT arrays with varying characteristic features and the study of field emission properties of these samples. 


\subsection{Controlled synthesis of CCNT arrays}

CCNT arrays on platinum wire substrates were synthesized using the same experimental procedure mentioned earlier in the experimental chapter. However. to study the effect of density and morphological variations of CCNTs on the field emission characteristics, five different samples of CCNT arrays were synthesized. The variation in the density and aspect ratio was achieved with an addition of a two-step process with a change in the gas phase composition. while all the other conditions remaining same. Step 1 consists of carbon deposition using $1.35 \%$ methane in $200 \mathrm{sccm}$ of hydrogen followed by a deposition and etching with $1 \%$ methane in step 2 with varying time scales for each step. Filed and thermionic emission characteristics were measured following the same procedures mentioned in the experimental chapter.

\subsection{Results}

\subsubsection{CCNT arrays with varying aspect ratio and density}

Figure 5.1 shows the SEM images of first three samples of CCNT arrays grown on a platinum wire. The corresponding insets present the enlarged view of individual CCNT tips, which show the absence of any metal catalyst at the tip. The experimental conditions employed and the resulting CCNT characteristics for samples 1-3 were summarized in Table 5.1. Increase in the time of step 1 increases the density and length, with $\sim 80 \%$ of the CCNTs within a few $100 \mathrm{~nm}$ of their average length. Increasing the duration of step 2 increases the etching of the CCNTs and also reduces the density of CCNTs. Table 1 illustrates the experimental conditions, the resulting structural and the field emission properties of the three CCNT array samples used in this study. 
Experimental conditions consist of the growth process time and the microwave power employed for the synthesis of all five CCNT samples. Structural characteristics include the average length, estimated density, and the average radius of each CNP sample from SEM image analysis. The results section includes the maximum field enhancement factor $\left(\beta_{\max }\right)$, calculated $r_{0}$ (using the equation for $\beta$ ), the lowest measured turn-on electric field $\left(E_{T}\right)$, the maximum emission current $\left(I_{\max }\right)$ per single CCNT corresponding to threshold electric field of $3.6 \mathrm{~V} / \mu \mathrm{m}$.
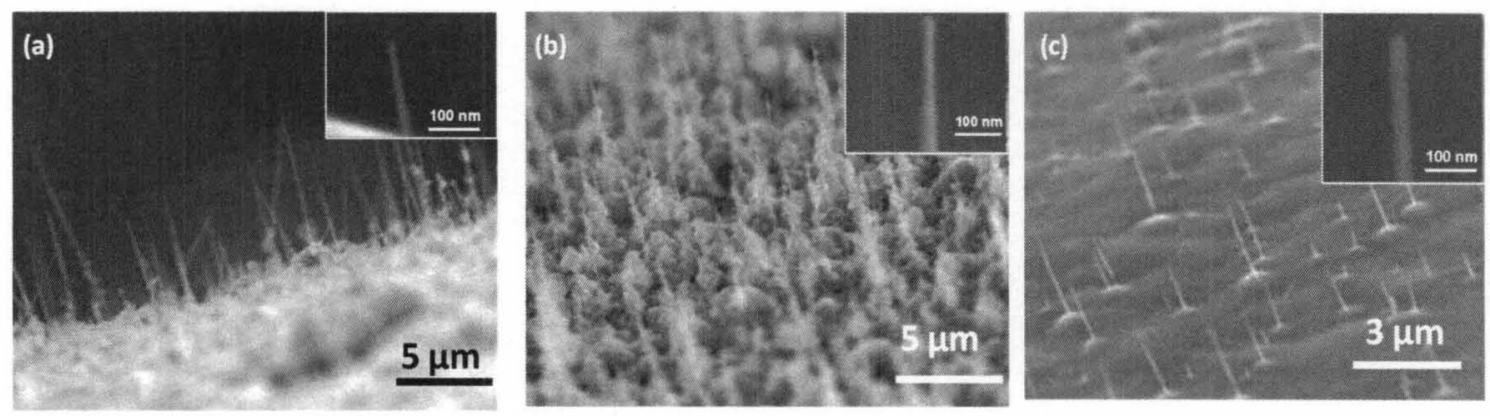

Figure 5.1 SEM images of array of CCNTs of (a) sample 1 (b) sample 2 and (c) sample 3, with insets showing the enlarged view of the corresponding CCNT tip. 
Table 5.1 Experimental conditions. the resulting structural characteristics, and the field emission properties of the three CCNT array samples used in this study

\begin{tabular}{|c|c|c|c|c|c|c|c|c|c|c|}
\hline \multirow[b]{2}{*}{\begin{tabular}{|l|l|l|} 
Sample I \\
H
\end{tabular}} & \multicolumn{3}{|c|}{$\begin{array}{l}\text { Experimetal } \\
\text { conditions }\end{array}$} & \multicolumn{3}{|c|}{ Structural properties } & \multicolumn{4}{|c|}{ Field emission properties } \\
\hline & Proce & (min) & \multirow[t]{2}{*}{$\begin{array}{l}\text { Power } \\
(\mathrm{W})\end{array}$} & \multirow[t]{2}{*}{$\begin{array}{l}\text { Density } \\
\left(10^{8} / \mathrm{cm}^{2}\right)\end{array}$} & \multirow{2}{*}{$\begin{array}{l}\text { Average } \\
\text { length } \\
\text { (1) }(\mu \mathrm{m})\end{array}$} & \multirow{2}{*}{$\begin{array}{l}\text { Average } \\
\text { radius (r) } \\
(\mathrm{nm})\end{array}$} & \multirow{2}{*}{$\begin{array}{l}\text { Calculated } \\
\text { radius }\left(\mathrm{r}_{0}\right) \\
(\mathrm{nm})\end{array}$} & \multirow{2}{*}{$\beta_{\max }$} & \multirow[t]{2}{*}{$\begin{array}{l}\mathrm{E}_{\mathrm{T}} \\
(\mathrm{V} / \mu \mathrm{m})\end{array}$} & \multirow[t]{2}{*}{$\begin{array}{l}I_{\max } \\
(\mu)\end{array}$} \\
\hline . & Step & Step & & & & & & & & \\
\hline 1 & 120 & 150 & 900 & 2.2 & 9 & 5 & 4 & 7600 & 0.66 & 520 \\
\hline 2 & 30 & 165 & 980 & 4.5 & 7 & 13 & 19 & 2313 & 1.5 & 320 \\
\hline 3 & 15 & 165 & 980 & 0.7 & 4 & 12 & 13 & 1324 & 2.3 & 200 \\
\hline
\end{tabular}

Figure 5.2 shows the SEM images of the individual CCNT of these three samples clearly depicting the variations in the wall structure and morphology. Sample 1 with the longest carbon deposition step, has CCNTs with the highest aspect ratio (Figure. 5.2a). moderate density and also the smallest tip radius estimated from SEM image characterization based on Figure 5.1 indicated in Table 5.1. Sample 2 has CCNTs with lower aspect ratio and larger tip diameter than sample 1 , in addition to the presence of carbon flakes along the length of each CCNT as depicted in Figure 5.2b. Sample 3 with longer etching step and a shorter deposition time resulted in CCNTs with the lowest aspect ratio and uneven etching as clearly seen in Figure 5.2 (c) 

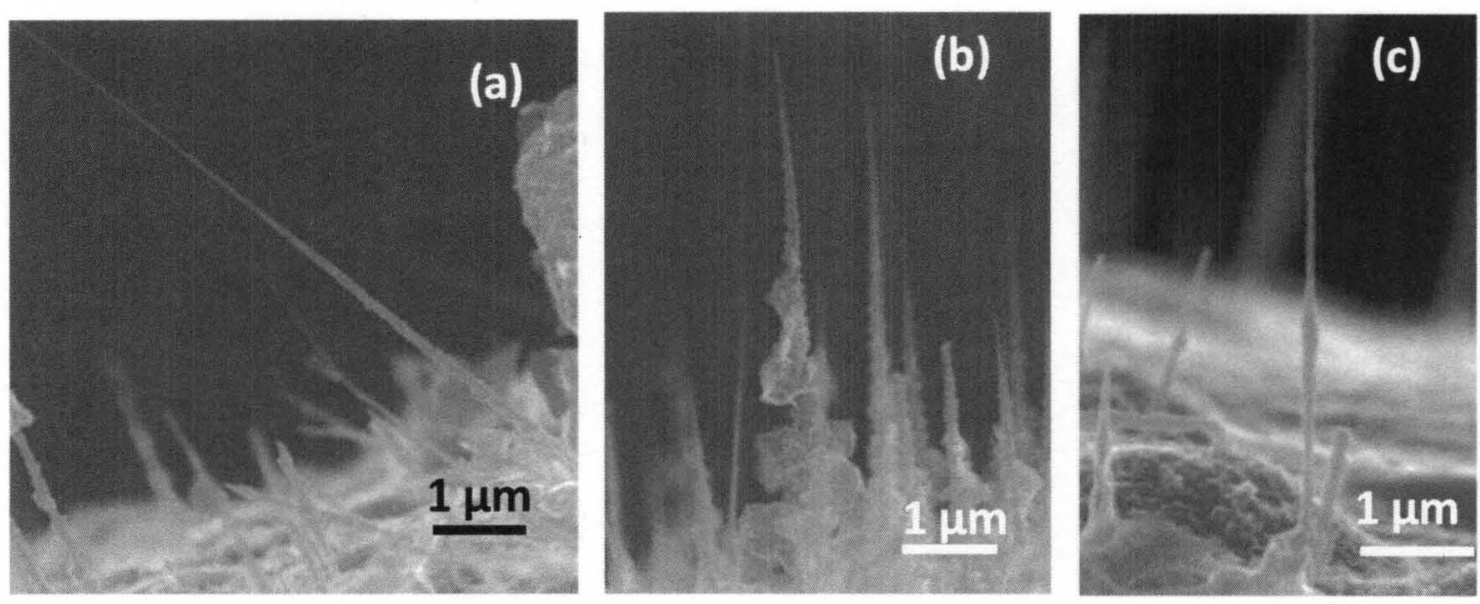

Figure 5.2 SEM images of the individual CCNT of (a) sample 1 (b) sample 2 and (c) sample 3, illustrating the variation in the wall structure and aspect ratio.

The last two samples, samples 4 and 5 were varied only in the CCNTs density while other structural characteristics such as length, radius of curvature and wall structure were maintained the same. Figure 5.3 shows the SEM images of (a) sample 4 and (b) sample 5 where the increased density of sample 5 is clearly seen. The structural and emission characteristics of both the samples are shown in Table 2.
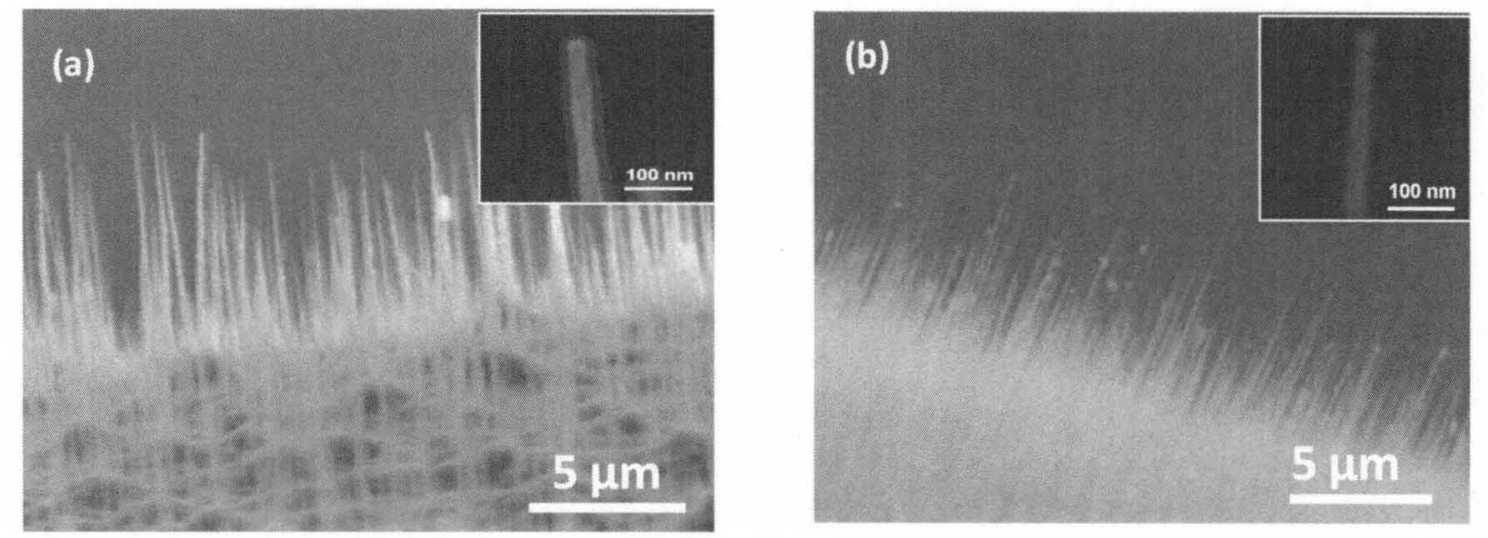

Figure 5.3 SEM images of CCNT arrays of (a) sample 4 (b) sample 5, with insets showing the enlarged view of the corresponding CCNT tip 
Table 5.2 Comparison of the resulting structural characteristics, and the field emission properties of the two morphologically similar but with different density CCNTs array samples.

\begin{tabular}{|l|l|l|l|l|l|l|l|}
\hline \multicolumn{7}{|l|}{ Structural properties } & \multicolumn{3}{l|}{ Field emission properties } \\
\hline Sample & Density & Average & Average & Calculated & $\beta_{\max }$ & $\mathrm{E}_{\mathrm{T}}$ & $\mathrm{I}_{\max }$ \\
& $\left(10^{8} / \mathrm{cm}^{2}\right)$ & length $(\mathrm{l})$ & radius $(\mathrm{r})$ & radius $\left(\mathrm{r}_{0}\right)$ & & $(\mathrm{V} / \mu \mathrm{m})$ & $(\mu \mathrm{A})$ \\
$(\mathrm{nm})$ & $(\mathrm{nm})$ & $(\mathrm{nm})$ & & & \\
\hline 4 & 15 & 7 & 12 & 11 & 2920 & 1.72 & 307 \\
\hline 5 & 20 & 7 & 11 & 11 & 1424 & 2.2 & 20 \\
\hline
\end{tabular}

\subsubsection{Field emission characteristics}

Of all the first three samples, CCNTs of sample 1 with its smallest tip radius, optimum density and highest aspect ratio resulted in enhanced field emission characteristics as described in the following analysis. Current (I) is measured as a function of applied electric field $(\mathrm{E}=\mathrm{V} / \mathrm{d})$ for sample 1 at each of the five inter-electrode distances (d) as shown in Figure 5.4a. According to the Folwer-Nordheim (F-N) equation the electric field $\left(E_{e f f}\right)$ at the tip of CCNT produces emission current density $(J)$ :

$$
J=\frac{A\left(E_{\text {eff }}\right)^{2}}{\phi} e^{-\left(\frac{B \phi^{3 / 2}}{E_{\text {eff }}}\right)}
$$


where $\phi$ is the work function of the emitter in electron volts (eV) (typically chosen to be 5 $\mathrm{eV}$ in the present case and other sp2 carbon materials $\left.{ }^{73}\right), A=1.56 \times 10^{-6} \mathrm{~A} \mathrm{~V}^{-2} \mathrm{eV}$, and $B=6.83 \times 10^{9} \mathrm{~V}(\mathrm{eV})^{-3 / 2} \mathrm{~m}^{-1}$. Figure $5.4 \mathrm{~b}$ shows the corresponding F-N plots of $\ln \left(J / E^{2}\right)$ vs. $l / E$ at each $d$ value. This plot represents the emission current region between the two knees of Figure 5.4a. The linearity of the plot is indicative of the field emission in the operating current regimes. The slope of this linear plot is given by $\left(\frac{B \phi^{3 / 2}}{\beta}\right)$, where $\beta$ is the field enhancement factor, defined as $E_{e f f}=\beta E_{a p p l}$ where, $E_{a p p l}$ is the applied electric field and $E_{\text {eff }}$ is the effective field at the emission point.

(a)

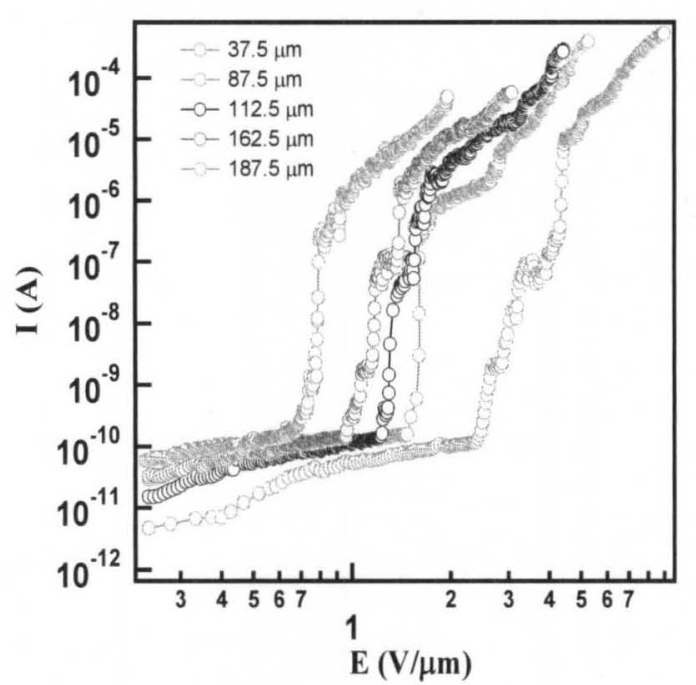

(b)

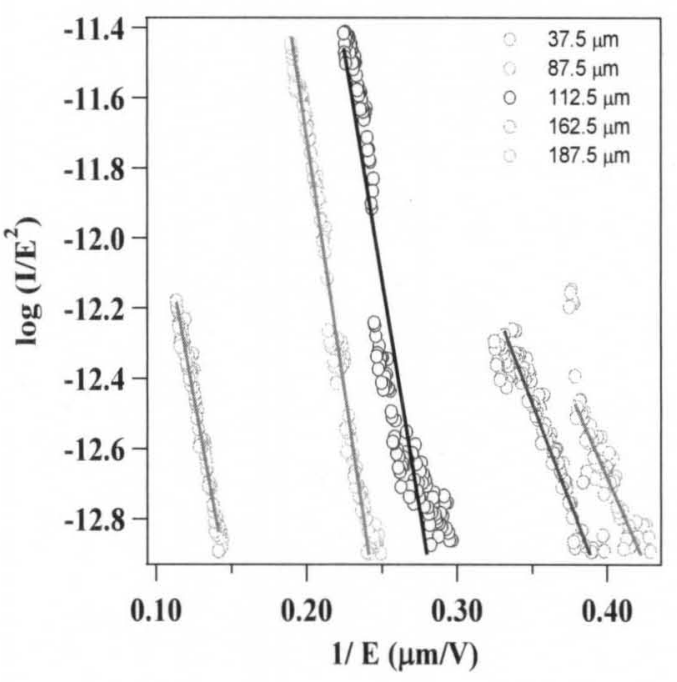

Figure 5.4 Field emission properties from the CCNTs showing the plots of (a) Current vs. the macroscopic electric field for various inter-electrode distances (d) for sample 1, (b) corresponding F-N plots. 
Figure 5.4a shows that the current begins to saturate at a second knee for sample 1 . This observed saturation may be associated with heat induced changes at the $\operatorname{tip}^{114}$. The maximum electric field reported for multiwall carbon nanotubes is $\sim 8 \mathrm{~V} / \mathrm{nm}$ before undergoing tip failure (due to deformation, evaporation, thermal runaway or arcing). The estimation of the current density in our samples is complicated by the curved platinum support. However, we have reached the effective electric field $\left(\beta E_{a p p}\right) \sim 8 \mathrm{~V} / \mathrm{nm}$ achieving current densities greater than $3 \mathrm{~A} / \mathrm{cm}^{2}$ at the second knee for the sample 1 at a distance of $187.5 \mu \mathrm{m}$, without experiencing thermal runaway.

Of the three samples, sample 1 has the highest $\beta$ value of 7,600 as shown in Figure 3 (c) which enables the CCNT array to continue to emit up to $d=187.5 \mu \mathrm{m}$ (for the maximum source voltage available). This high value of $\beta$ is due to a combination of factors such as small radius of curvature, high aspect ratio, moderate emitter density of CCNTs in sample 1 and the increased distance $(d)$. In the previous study on CCNTs terminated with nickel catalyst particles, the field enhancement factor as low as 80 have been reported ${ }^{73}$. The value of $\beta$ for samples 2 and 3 ranges from 2,000 - 3,000. Sample 3 has the poorest emission properties of three samples due to the formation of amorphous carbon along the side walls of CCNT resulting from the prolonged etching which minimizes the edge plane emission sites ${ }^{115}$. The value of $\beta$ determined by field emission study is large compared to the geometrical enhancement factor given by $h / r$, where $h$ is the length of the CCNT and $r$ is the radius at the tip. This deviation was also observed earlier for highly dense carbon nanotube arrays which demonstrated that $\beta$ depends on the inter-electrode distance $(d)$ and other factors such as tip radius and aspect ratio ${ }^{96}$. In the present case this discrepancy can be attributed to the structural characteristics of these 
conical morphologies with open edges on the outer surface acting as emission sites. The effects of the surface adsorbates also cannot be ruled out ${ }^{56}$. Figure $5.5 \mathrm{~b}$ shows that turnon electric field ( $E_{\mathrm{T}}$, electric field corresponding to minimum detectable emission current) decreases with increasing distance $(d)$ for all three samples reaching a value as low as 0.7 $\mathrm{V} / \mu \mathrm{m}$ for sample 1 at the maximum separation distance, which can be accounted for the high $\beta$ value. This low turn-on electric field value is comparable to the best values reported for SWNTs and MWNTs ${ }^{62-63}$.

(a)

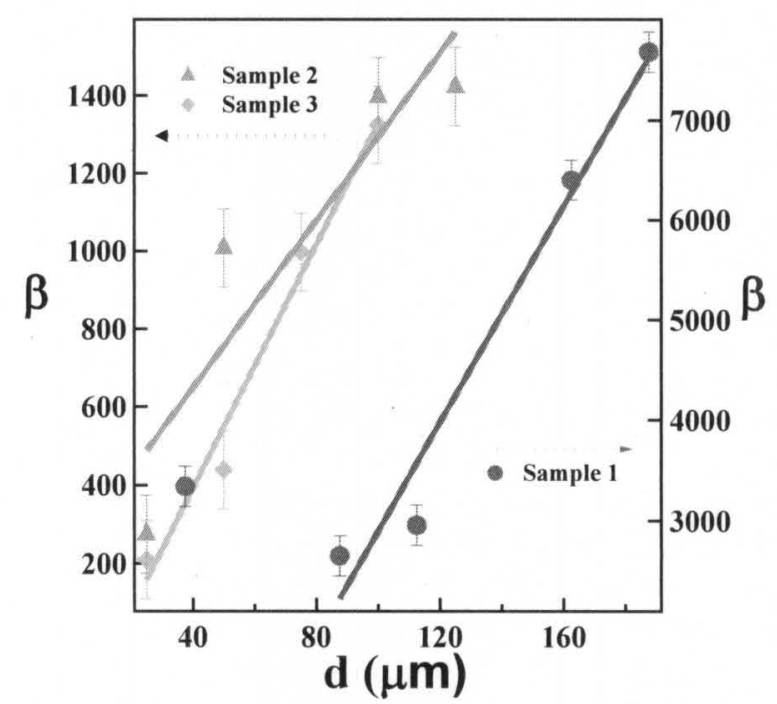

(b)

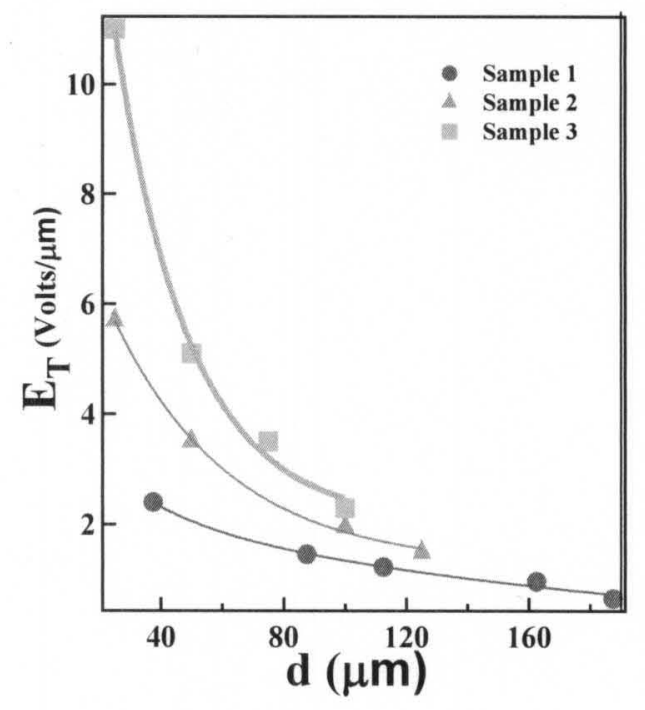

Figure 5.5 Plots of (a) field enhancement factor $\beta$ and (b) turn-on electric field as a function of distance for samples 1-3 
The emission properties of samples 4 and 5 are shown in Figure 5.6. It is very clear from the figure and the table 5.2, that sample 4 has superior emission properties compared to sample 5. This can be attributed to the reduced field screening affect from the neighboring emitters due to lower density in the case of sample 4 compared to 5 .

(a)

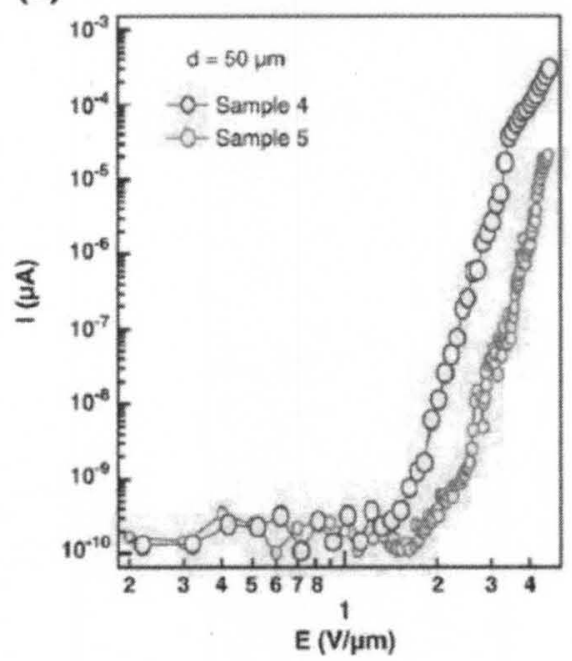

(b)

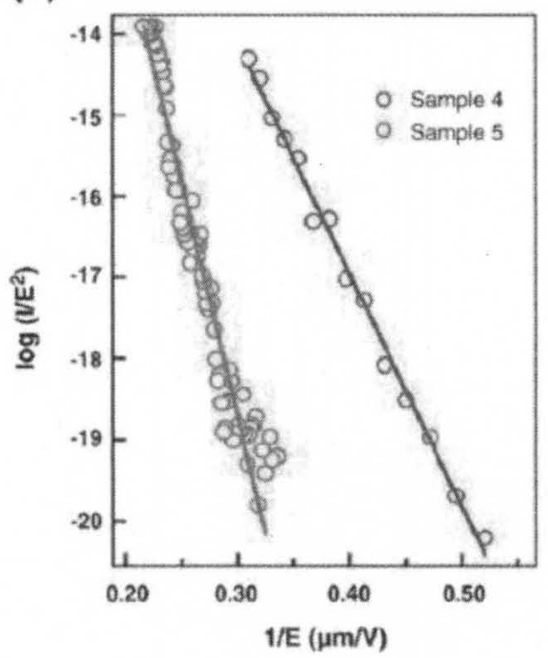

Figure 5.6 Field emission properties from the CCNTs showing the plots of (a) Current Vs. the macroscopic electric field for sample 4 and sample 5, (b) corresponding F-N plots.

\subsubsection{Thermionic emission (TE) characteristics}

Here we attempt to study the work function values of these novel conical carbon structures CCNTs, which was not been studied so far. In addition, this study ensures the accurate understanding of TE from the hybrid structure involving these CCNTs and diamond crystals. The CCNTs on platinum wire and graphite foil were studied using both thermionic emission and UPS measurements 
As synthesized CCNT arrays grown on platinum wire substrate as described above was used as substrate for determining the thermionic emission properties. In the present case field enhanced thermionic emission (FETE) was chosen to prevent any space charge problems as discussed earlier. The range of voltage swept in the present case was chosen as $0-500 \mathrm{~V}$, such that major contribution to emission current would be from thermionic emission. It is in this region the effect of temperature on emission current will be apparent. However, when the voltage was increased to $1000 \mathrm{~V}$, field emission becomes predominant process of emission due to field enhancement effect. In this region, the current increases exponentially with the field as discussed in previous section.

The modified equation includes Schottky effect and governs the FETE process, called Richardson-Dushman equation expressed as:

$$
J=J_{0} e^{-\left(\frac{C \sqrt{E}}{K T}\right)}
$$

$J_{0}$ is referred to as the zero-field TE current density and it expressed according to Richardson as:

$$
J_{0}=A T^{2} e^{-\left(\frac{\Phi}{k T}\right)}
$$

And

$$
C=\sqrt{\frac{e^{3}}{4 \pi \varepsilon_{0}}}
$$


As shown below, Figure 5.7 illustrates the measured current-voltage characteristic plots of FETE from CCNT array on platinum wire substrate at various temperatures.

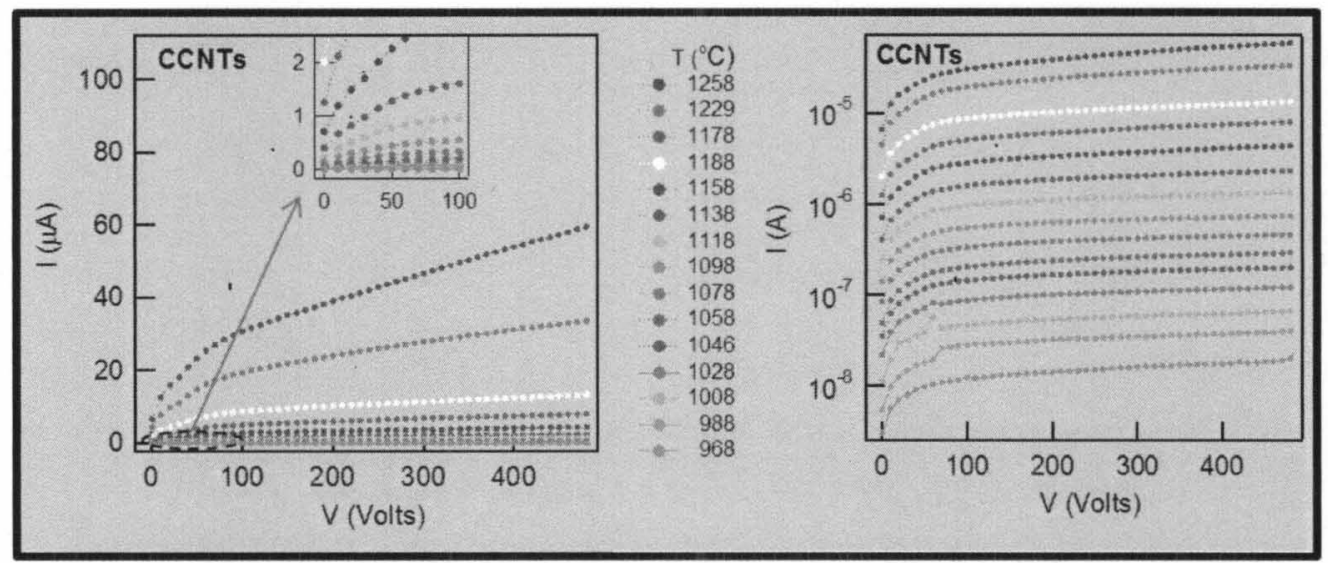

Figure 5.7 FETE plots of $I-V$ characteristics from CCNT arrays at various temperatures (a) In linear scale, with enlarged view in inset and (b) Ln-linear scale.

From thermionic $I-V$ characteristic, zero-field current density $J_{0}$ at different temperatures can be determined from the intercepts of $\operatorname{LnI}$ vs $\sqrt{V}$ curves as shown in Figure 5.8a and b. Using Richardson equation and by fitting experimental curves $\operatorname{Ln}\left(\frac{J_{0}}{T^{2}}\right)$ vs $\frac{1}{T}$ as shown in Figure $5.8 \mathrm{c}$, the values of work function value of CCNT arrays can be determined. The linearity of this plot indicated that the collected electrons are due to TE. TE behavior is characterized by a constant slope in Richardson plot and slope is proportional to the work function. Thus a work function value of $4.2 \mathrm{eV}$ was obtained from CCNT arrays on platinum wire. 


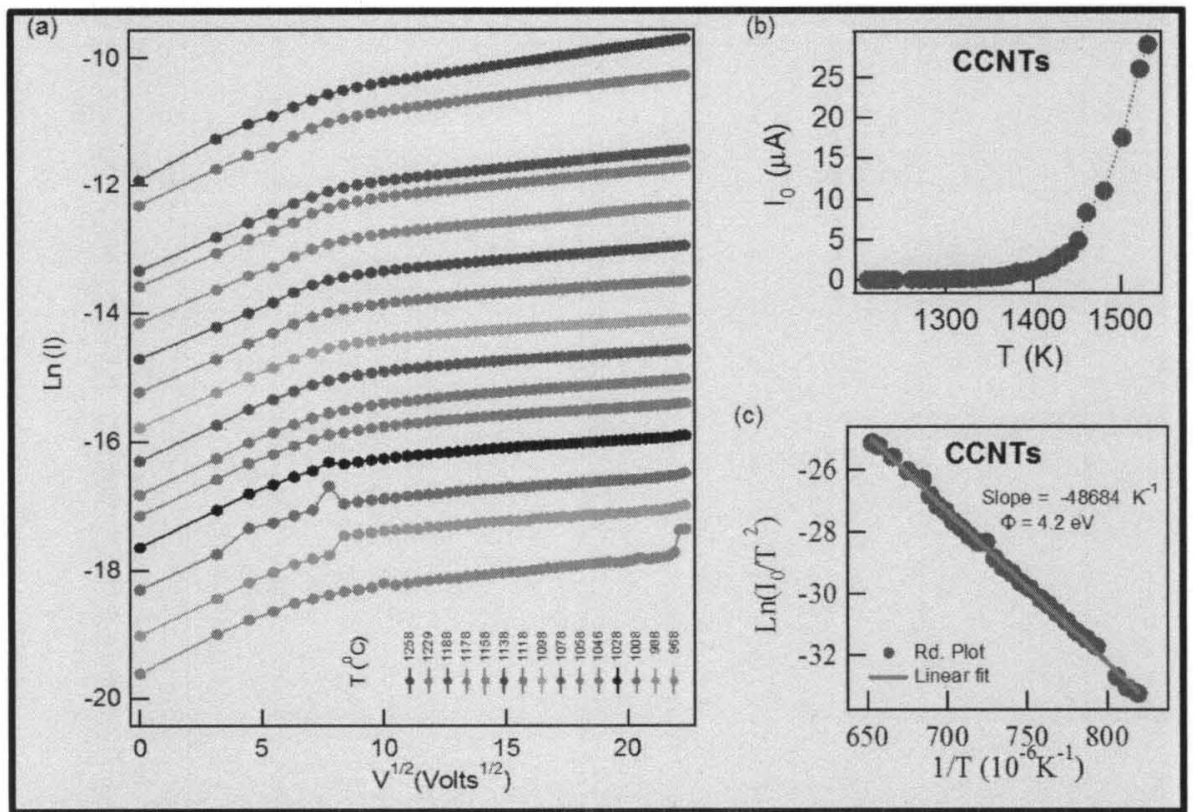

Figure 5.8 TE characteristics of CCNTs (a) $\operatorname{Ln}(I) \mathrm{vs} V^{1 / 2}$, (b) Richardson plot of $I \mathrm{Vs} \mathrm{T}$ and (c) linear fitting of $\operatorname{Ln}\left(\frac{I_{0}}{T^{2}}\right)$ vs $\frac{1}{T}$ (straight line) to the experimental data (solid circles).

Similarly, as synthesized CCNT arrays grown on graphite foil using similar experimental conditions mentioned in chapter 4 was used as the substrate for determining the thermionic emission properties. A work function value of $4.0 \mathrm{eV}$ was obtained from these CCNT arrays from FETE characteristics as shown in Figure 5.9. The observed lower work function values of 4.0 - $4.2 \mathrm{eV}$ for CCNTs compared to CNTs with work function values $4.6-5 \mathrm{eV}^{27}$ can be attributed to the presence higher aspect ratios leading to a greater field enhancement and presence of high density of edge planes in the case of CCNTs. Similarly, higher aspect ratios of CCNTs on graphite foil substrates compared to 
those on platinum wire substrates can be attributed to the observed lower work function values from graphite foil substrate..

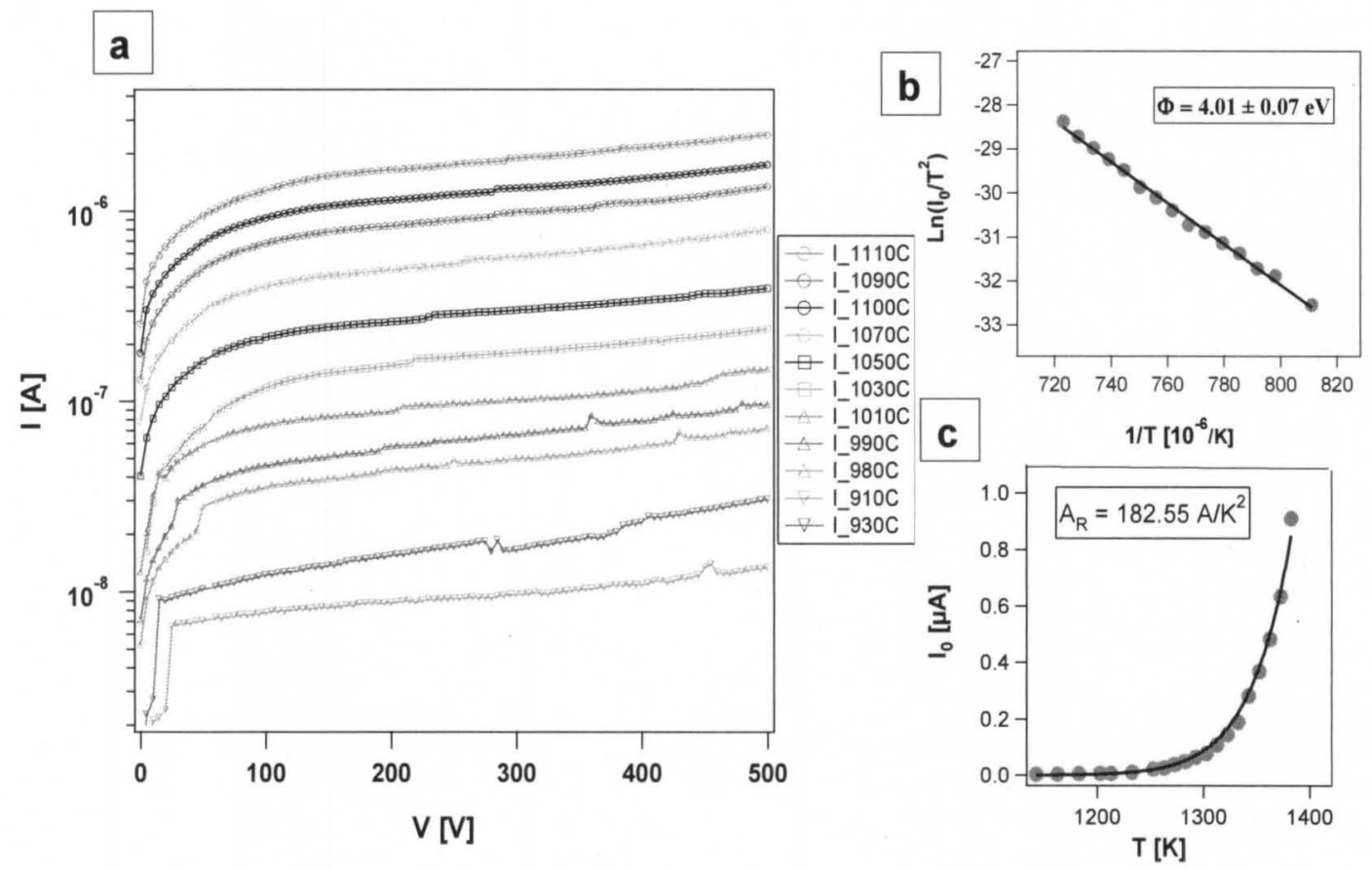

Figure 5.9 TE characteristics of CCNTs (a) $\operatorname{Ln}(I)$ vs $V^{1 / 2}$, (b) Richardson plot of $I$ Vs T and (c) linear fitting of $\operatorname{Ln}\left(\frac{I_{0}}{T^{2}}\right)$ vs $\frac{1}{T}$ (straight line) to the experimental data (solid circles).

\subsubsection{Ultraviolet photo spectroscopy (UPS) studies}

Work function values can also be determined using UPS. As synthesized CCNT arrays on platinum wire and graphite foil substrates were used as the samples. These samples for the measurements with the active material are in electrical contact with gold film sputtered on silicon wafer to equilibrate the Fermi levels $\left(E_{F}\right)$. A part of the silicon wafer sputtered with gold was used for the sample and the other half for acquiring the 
reference gold spectra. Double side copper tape was used for the electrical contact between silicon substrates with diamond films and the underlining gold film on copper foil. The measurements were performed using He-I $(21.23 \mathrm{eV})$ and He-II $(40.81 \mathrm{eV}) \mathrm{UV}$ excitations. A stable bias was provided to avoid the instrumental cutoff in the lens system of the analyzer at low kinetic energy (KE) for all the UPS spectra measurements. The external bias that was measured and the spectra were shifted back to zero-bias position through post acquisition data processing. The calibration of the UPS spectrometer was performed by measuring and validating the absolute position of the Fermi level of the gold sample using He-I, He-II excitations at $21.2 \mathrm{eV}$ and $40.83 \mathrm{eV}$.

As the active sample is in electrical contact with the reference gold film, the sample also had the same Fermi level as of the gold. Figure 5.10 shows the low kinetic energy part of the He-I emission spectra of CCNT arrays on a) platinum wire and b) foil substrate. Fermi level of the spectra is assumed to be aligned to that of gold Fermi as CCNT array substrates are in good electrical contact with underlining gold film. The work function is then determined from the intersection of low-KE cut off tail with the background level as shown in the insets of Figure $5.10 \mathrm{a}$ and $5.10 \mathrm{~b}$ and a value of $4.4 \mathrm{eV}$ was obtained from CCNT arrays on platinum wire and graphite foil substrates, which also validate the work function values obtained using TE measurements. 

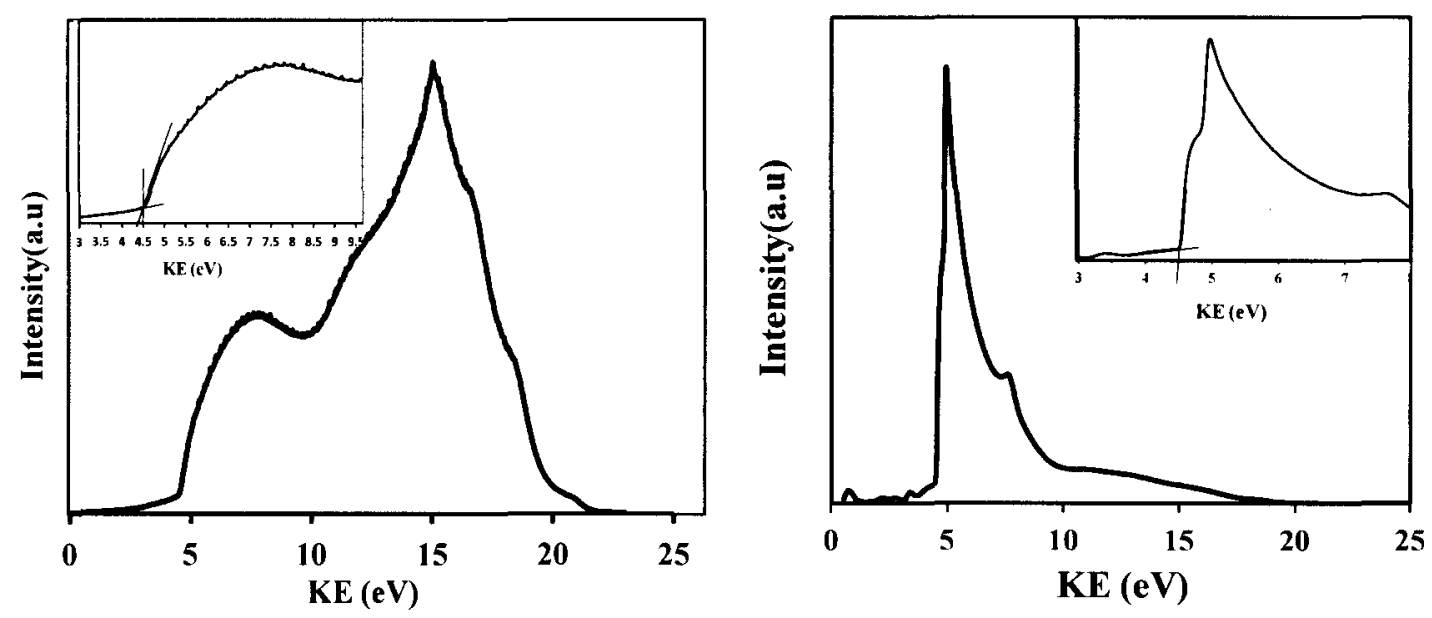

Figure 5.10 Low kinetic-energy He - I (21.23 eV) spectra of CCNT arrays on (a) platinum wire and (b) graphite foil substrate with insets showing low kinetic cut-off energy positions indicative of work function

\subsection{Summary}

In this chapter. CCNT array samples with different densities, radii of curvatures, lengths and wall structures were successfully synthesized by varying the critical growth parameters. Field emission studies on these conical structures resulted in a turn-on electric field as low as $0.7 \mathrm{~V} / \mu \mathrm{m}$ and field enhancement factor as high as 7,600 . It was also shown that not only the characteristic features of emitter such as high aspect ratio, small radius of curvature of the tip, enhanced the field emission properties, but also optimum emitter density plays an important role in reducing the field screening effect. With controlled aspect ratio, density, and uniformity; CCN'Ts can be turned into potential field emitters and next generation cold cathode field emitters. Thermionic emission and ultraviolet photospectroscopy studies on CCNTs have resulted in work function values as low as $4.0 \mathrm{eV}$. compared to pristine CNTs exhibiting work function values $>4.5 \mathrm{eV}$. 


\section{CHAPTER 6}

\section{DIAMOND TIPPED AND COATED CCNT ARRAYS}

\subsection{Introduction}

As discussed earlier (Chapters 2, 4) CCNTs represent a new morphological manifestation of carbon nanotubes with unique characteristic features exhibiting a tapered geometry with the entire surface consisting of graphitic edge planes. Due to their unique features, these CCNTs could serve as the template for the individual nanodiamond tip

arrays for biological and electronic applications ${ }^{80,81}$. Though diamond nucleation studies on MWNT and SWNT structures have been reported, the pathway for nucleation is not completely understood due to variation in the proposed mechanistic aspects ${ }^{87,91}$. Moreover the study of diamond nucleation on CCNTs exhibiting unique morphological characteristics compared to MWNT or SWNT has never been studied.

This chapter describes the synthesis and structure of diamond tipped and diamond coated CCNTs. The growth of the diamond crystals on CCNTs takes place under similar experimental condition used for the growth of CCNTs on platinum wire, except variation in the placement of the substrate to create the necessary temperature, radical and neutral species environment for the diamond nucleation. Similar experimental conditions mentioned in chapter 3 were employed, along which the effect of some pretreatment methods on the nucleation and growth of diamond crystals has also been studied here. Due to their unique hybrid architecture, very different from that of MWNT or SWNT, the 
nucleation and growth mechanism was studied to understand the underlining factors and to be able to control size and density of the diamond crystals.

\subsection{Pretreatment procedures}

CCNT arrays on platinum wire substrates were synthesized following the experimental conditions mentioned in chapter 3. Nucleation and growth of diamond on these CCNT arrays is carried out using the similar experimental procedures as mentioned earlier with varying time scales. In addition to this, two different pretreatment methods are employed here to enhance the nucleation density of diamond crystals using diamond (micron and submicron particle) and boron carbide $(0-7 \mu \mathrm{m})$ powder dispersions (acetone). 1. Immersion of CCNT arrays in a sonicating dispersion bath for few seconds. 2. Electropheretic seeding of CCNT arrays using the same dispersion solutions for few minutes. Graphite plate is used as cathode and platinum wire with CCNT arrays as anode with a bias of $40 \mathrm{~V}$ applied using a potentiostat. The array substrates were thoroughly washed with acetone after the pretreatment

\subsection{Results}

\subsubsection{Structural characterization}

Figure 6.1a shows the scanning electron microscope (SEM) images of an array of as-synthesized CCNT arrays with single CCNT shown in Figure 6.2b. 

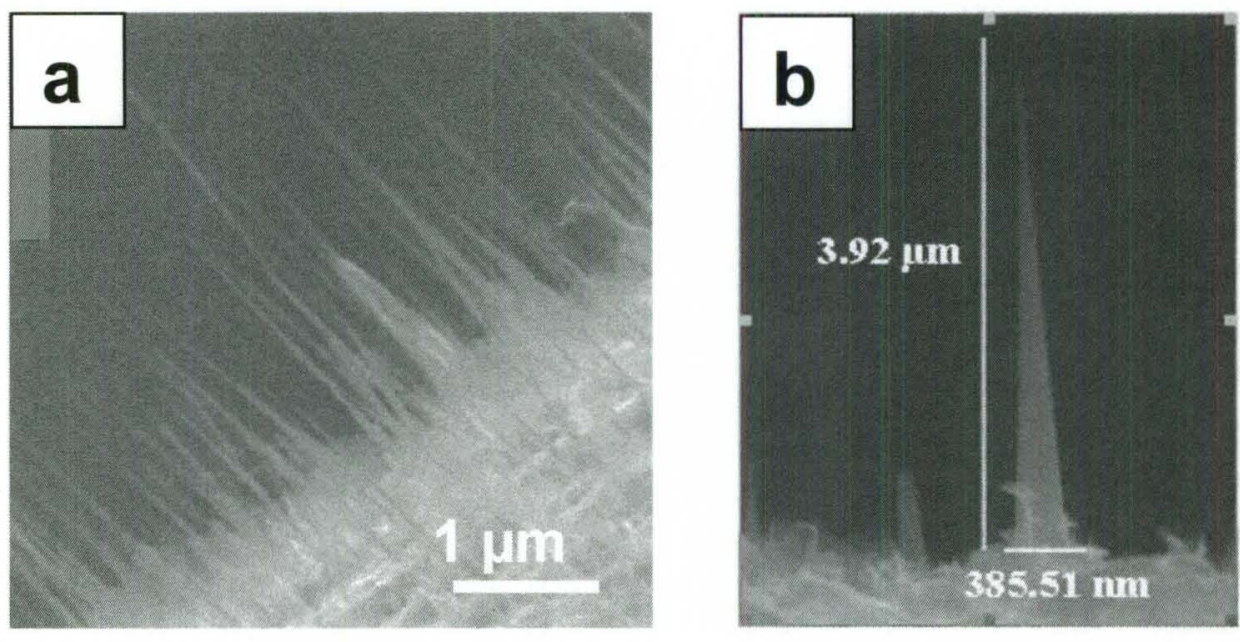

Figure 6.1 SEM image of (a) an array of as synthesized CCNTs on platinum wire substrate, (b) individual CCNT exhibiting conical geometry

The diamond nucleation and growth experiments over short (20 min) durations on these as-synthesized CCNT arrays resulted in selective nucleation and growth of submicrometer-size diamond crystals (200-500 $\mathrm{nm}$ in diameter) at their tips shown in Figure 6.2a. Increase in the size of the crystal was observed with the increase in the duration of the growth, leading to diamond crystals as large as upto 1-2 $\mu \mathrm{m}$ for duration of about $3 \mathrm{~h}$ (Figure.6.2b). Some of the other observations noticed include; 1. The rough faceting morphology of the diamond crystal along with selective growth only at the tips of CCNTs and 2. The conical morphology of CCNT structures was lost resulting in straight CNTs, but with no appreciable change in their lengths seen in Figure 6.2c. Both these observations were interesting and unexpected which are discussed in detail later in this chapter. Raman spectra (RS) were obtained from various regions on this diamond-tipped CCNTs substrate using a $633 \mathrm{~nm}$ laser with a spot size of $1-2 \mathrm{~mm}$. Figure $6.2 \mathrm{~d}$ is a typical 
spectrum showing a peak at $1332 \mathrm{~cm}^{-}$, confirming the existence of sp3 phase of the diamond crystal.
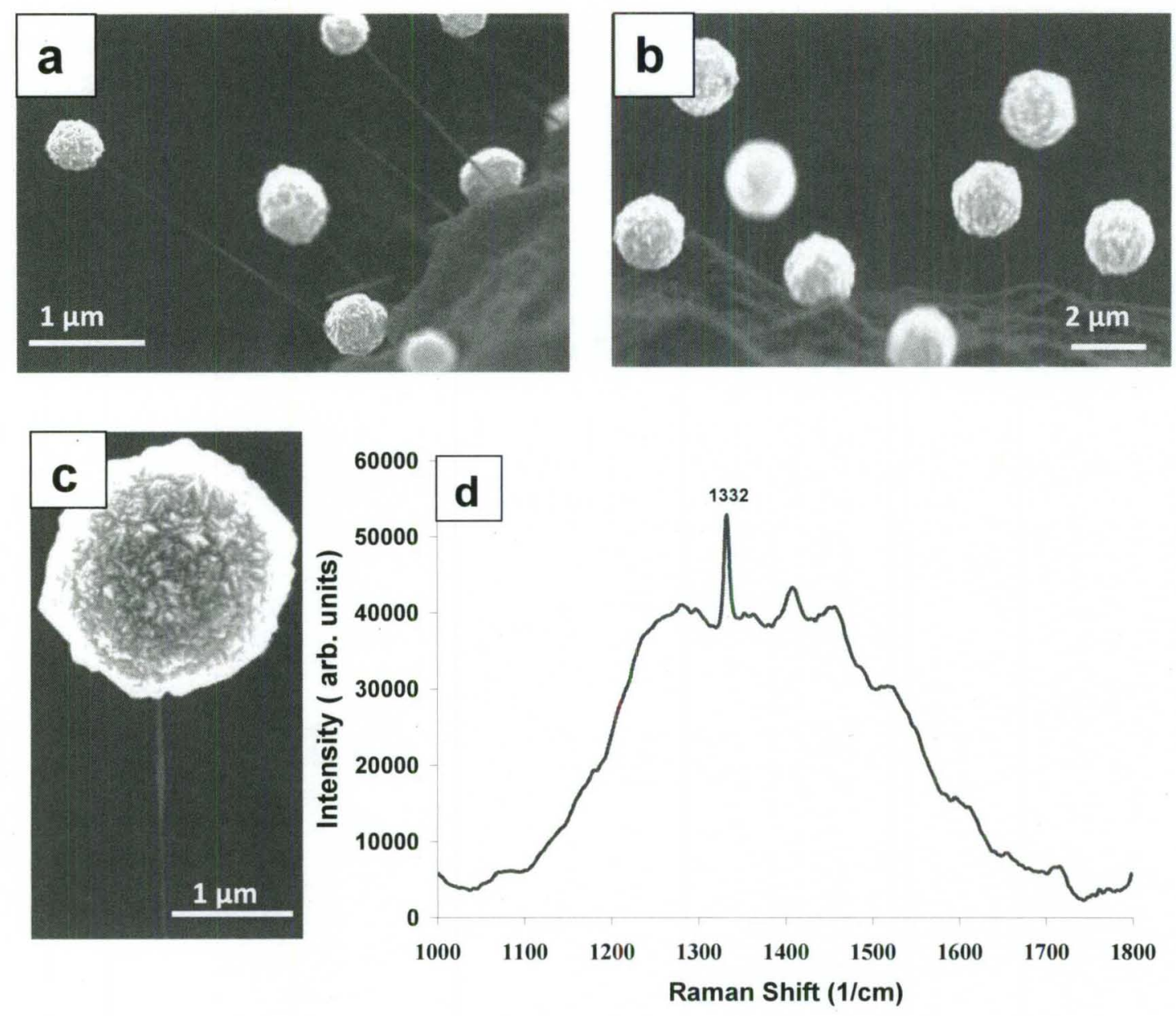

Figure 6.2 SEM images of (a) selective growth of sub-micron scale diamond crystals on tips of CCNTs grown for $20 \mathrm{~min}$, (b) micron scale diamond crystals with growth duration of about $3 \mathrm{hr}$, (c) an individual CCNT etched to CNT with rough faceting diamond crystal at the tip and (d) Visible raman spectrum showing the diamond characteristic peak at $1332 \mathrm{~cm}^{-1}$ 
The results of the pretreatment involving the immersion in the dispersion bath of micron and submicron sized diamond powder for $20 \mathrm{sec}$ are shown in Figure 6.3. Increase in the density of diamond crystals was observed in both the cases. However, with micron $(0-2 \mathrm{~mm})$ diamond powder, the increase in density was observed only at the tips of CCNTs as shown in the Figure 6.3a. On the other hand with sub micron $(0-250$ nm) diamond powder, nucleation of diamond was not only observed at the tips, but also along the length of CCNTs (Figure 6.3b). Further increase in the density of the diamond crystals was observed when the CCNT arrays were pretreated by electrophoretic seeding. In the case of seeding with micron diamond bath, nucleation was observed along the length of CCNTs in addition to the tips as shown in Figure 6.3c. However, seeding with sub micron diamond bath resulted in complete coating of the underlining CCNTs with nanocrystalline diamond as shown in Figure 6.3d. Figure 6.3e shows a typical UV Raman spectrum obtained using a $325 \mathrm{~nm}$ laser with a $1-2 \mathrm{~mm}$ spot size on the sample shown in Figure $7 \mathrm{~d}$, which shows a peak at $1332 \mathrm{~cm}^{-1}$ confirming the presence of diamond crystals, as well as a peak at $1584 \mathrm{~cm}^{-1}$ characteristic of graphite. 

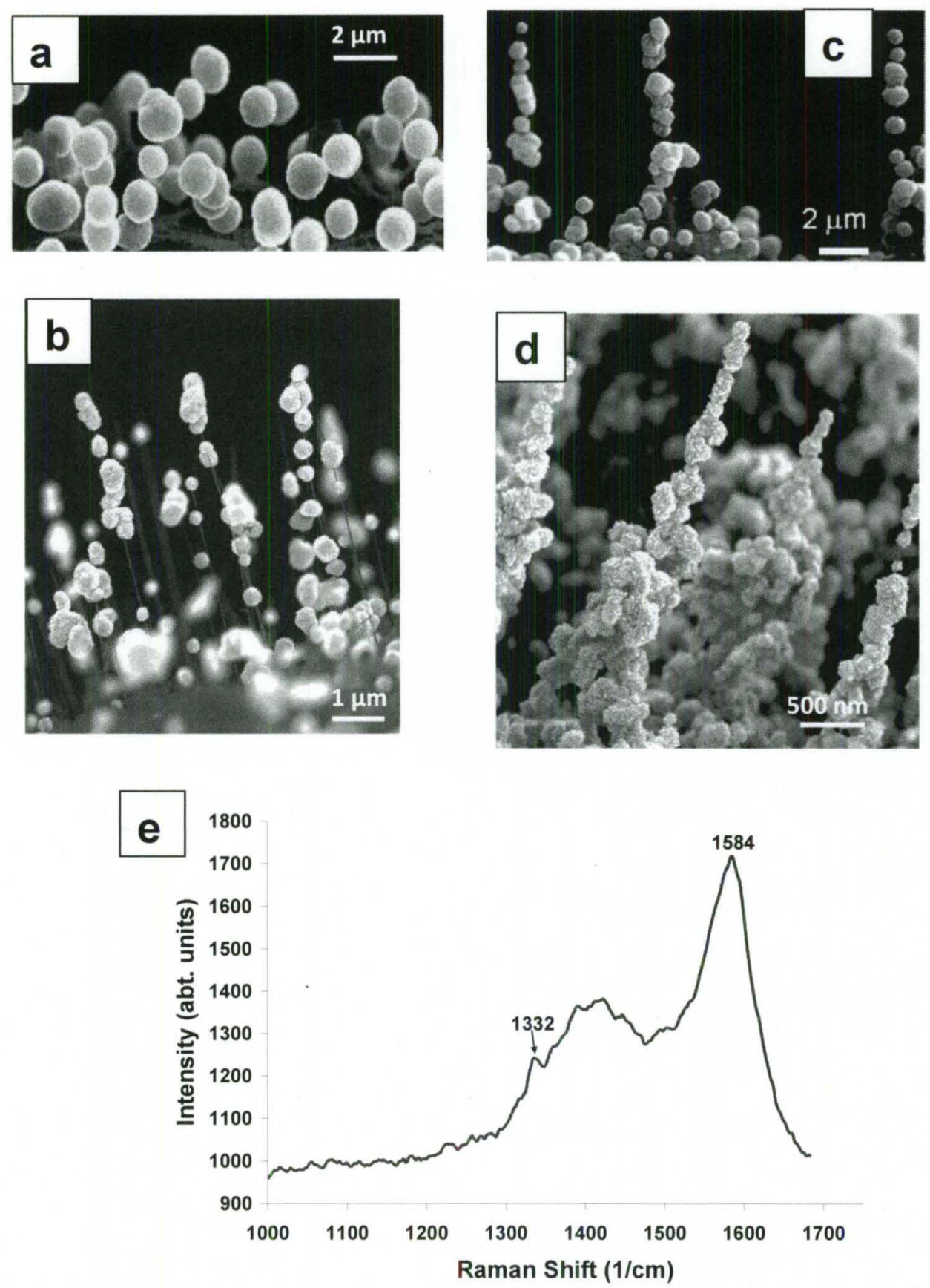

Figure 6.3 SEM images showing diamond nucleation on CCNT as a result of pretreatment involving sonic dispersion bath of (a) micron-scale diamond powder, (b) submicron-scale diamond powder, pretreatment involving electrophoretic seeding in dispersions of (c) micron-scale, (d) sub-micron scale diamond powder and (e) UV raman 
spectrum confirming the presence of diamond exhibiting diamond characteristic peak at $1332 \mathrm{~cm}^{-1}$

\subsubsection{Thermal run away}

As mentioned earlier, the diamond crystals grown at the tips of CCNT exhibit high amount of rough faceting as shown in Figure 6.2c. A bright field transmission electron microscope (TEM) image shown in Figure 6.4a also confirms the rough faceting of the crystals which could be due to high density of defective growth regions. But, it certainly does not show it as if it is composed of many crystals coming together to form a ball shaped cluster. The observed rough faceting or high levels of defective growth of crystals growing on the tips of CCNTs could probably be explained with a thermal runaway scenario. The temperature of the growing diamond crystal could rise with increase in crystal size if the nanotube cannot adequately support the transport of heat generated from the exothermic hydrogen recombination at the growing crystal surface $\mathrm{e}^{116}$. The schematic of the model in shown in Figure $6.4 \mathrm{~b}$. A simple thermal transport model yields the following;

$$
\Delta T=\frac{J \cdot A_{d} \cdot \Delta H}{k \cdot A_{t} \cdot L}
$$

Where, $\Delta T$ is the change in temperature, $\mathrm{J}$ is the hydrogen flux, $\mathrm{A}_{\mathrm{d}}$ is the area of the diamond, $\Delta H$ is the total enthalpy, $\mathrm{k}$ is the thermal conductivity, and $A_{t}$ is the cross sectional area and $\mathrm{L}$ is the length of the carbon nanotube. The heat of the recombination reaction, $\mathrm{H}+\mathrm{H}=\mathrm{H}_{2}$, was taken as $\Delta \mathrm{H}_{\mathrm{r}}=-444 \mathrm{~kJ} / \mathrm{mol}$ of $\mathrm{H}_{2}{ }^{117}$. The flux of hydrogen atom bombardment was calculated to be approximately $1 \times 10^{-4} \mathrm{~mol} / \mathrm{cm}^{2} \cdot \mathrm{s}$, which is 
comparable to reported values ${ }^{117}$. A standard planar graphite conductivity value of 1000 $\mathrm{W} / \mathrm{m} \cdot \mathrm{K}$ was applied to a nanotube with a $20 \mathrm{~nm}$ outer diameter (OD) and a $10 \mathrm{~nm}$ inner diameter (ID) and length of $10 \mu \mathrm{m}^{118}$. It can be observed from Figure $6.4 \mathrm{c}$ that as the diamond crystal increases in size, the temperature increases parabolically, leading to a thermal runaway scenario. Once heat buildup occurs, the temperature of the crystal rises quickly which could lead to defect generation which further roughens up the crystal faceting. 

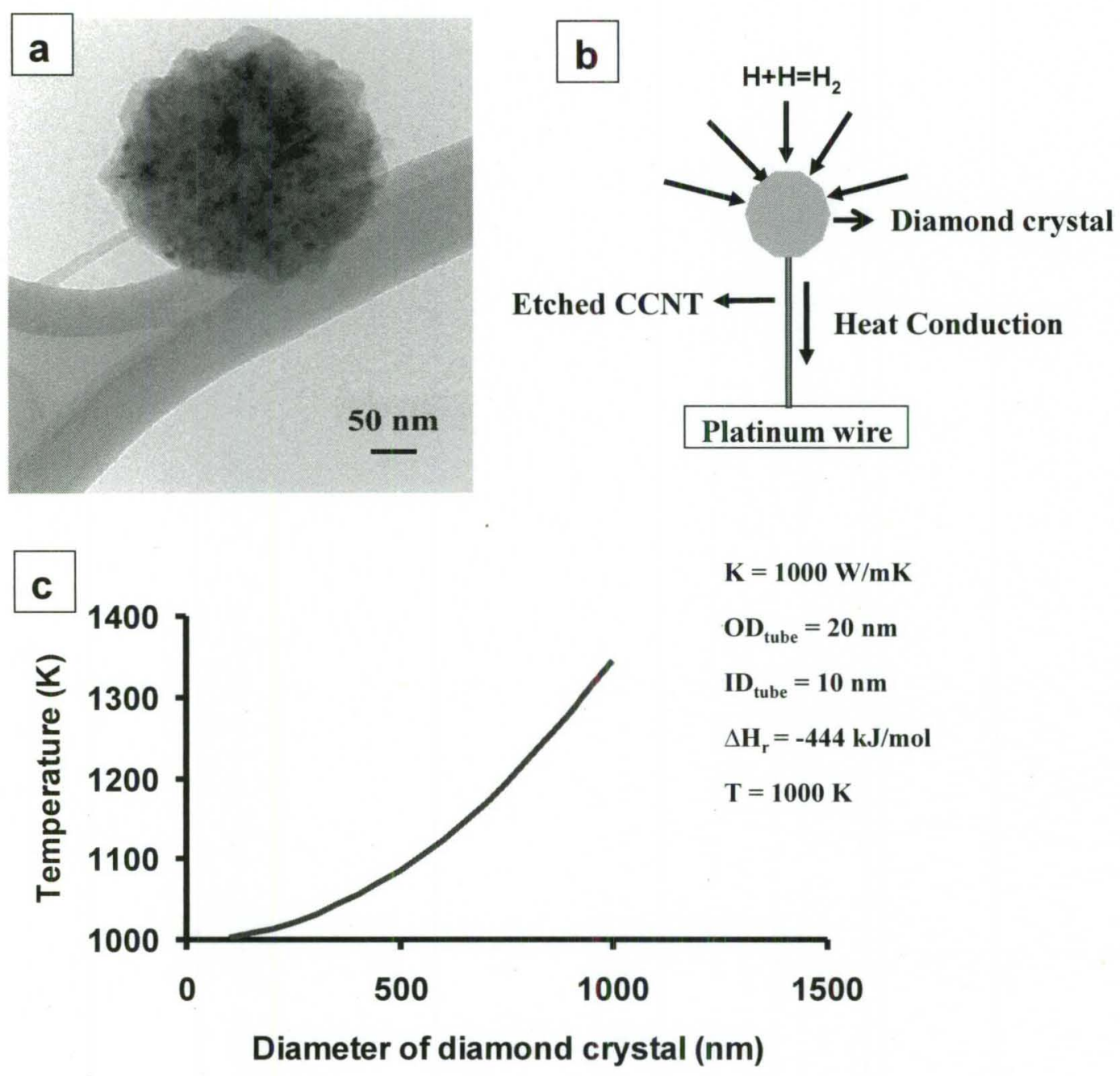

Figure 6.4 (a) Bright field TEM image of the individual diamond crystal at the tip of etched CCNT, (b) a schematic illustration of thermal run away scenario of the diamond crystal at the tip of the nanotube and (c) plot showing the estimated temperature rise during diamond growth as a function of size of the diamond crystal

\subsubsection{Hydrogenation of CCNT}


As mentioned earlier, during the growth of the diamond crystals, the CCNTs were etched to CNTs while the lengths were being preserved. In order to further study the etching behavior, as synthesized CCNTs were exposed to hydrogen plasma for short time (20 min). It was evident from the Figure 6.5 that the as synthesized CCNTs (Figure 6.5a) were etched to straight MWNTs (Figure 6.5b) with uniform diameter after the hydrogen plasma treatment. Figure $6.5 \mathrm{~b}$ also shows some intermittent results of etching where some CNPs were etched to different thicknesses along their lengths.
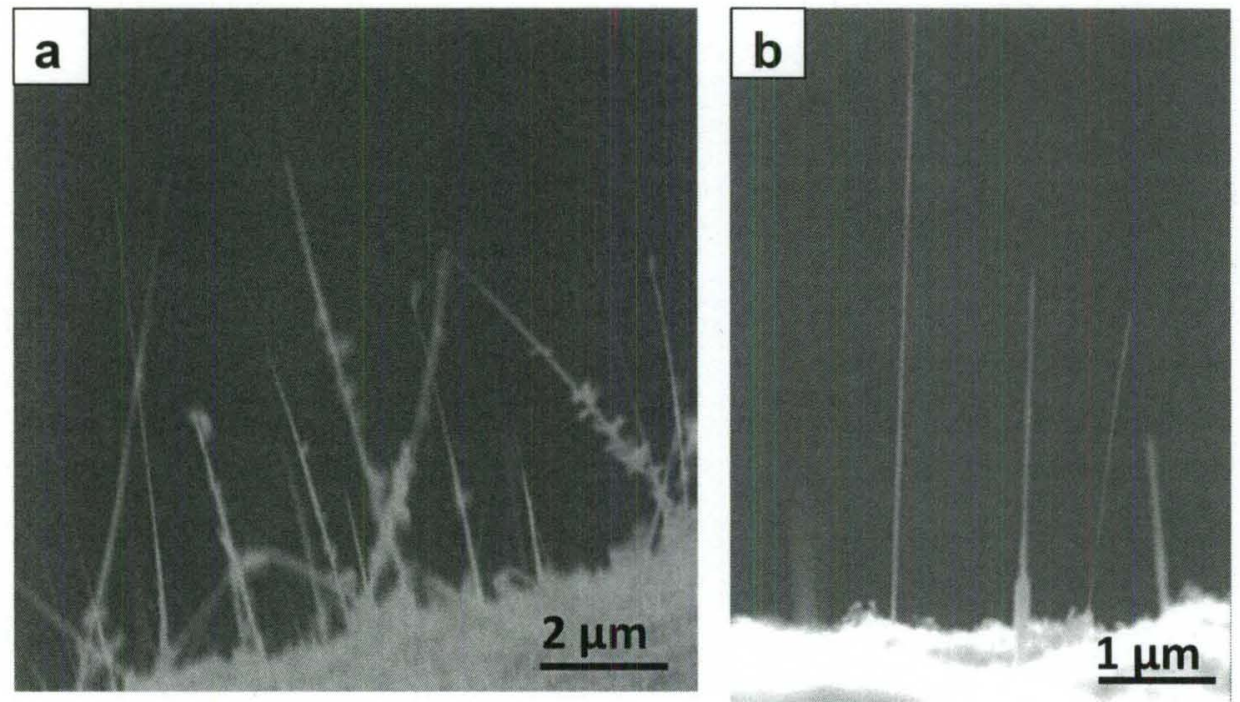

Figure 6.5 SEM images of an array of (a) as synthesized CCNTs with tapered morphology with various lengths and (b) CCNTs etched to CNTs after hydrogen plasma etching.

However, it was observed that the lengths of CCNTs were not affected by hydrogen plasma treatment. High resolution (HR) TEM studies were performed to understand the plausible reasons for the preservation of lengths by characterizing these 
structures at various stages of etching. The SEM images of the individual as synthesized CCNT and MWNTs as a result of etching were shown in Figures 6.6a and 6.6b with insets showing the HRTEM images of their respective wall structures. It was observed that the graphitic edge planes of the CCNT were etched until only the basal planes (MWNT) were exposed as shown in the insets. From these observations, the presence of aggressive etching conditions (due to bombardment by hydrogen radicals) prevailing on the surface of CCNTs during the diamond growth could be attributed to the observed transformation in the morphology. However, the etching of entire CCNT surface except the tip was more surprising because the reactive atomic hydrogen should etch down the length of the CCNT consisting of exposed graphitic edges at the tip ${ }^{84}$. TEM observations were performed on one of the partially etched CCNT to observe the wall structure at the tip during the etching. The bright field TEM image in Figure 6.6c shows a etched CCNT with a inset showing HRTEM image of the closure of the graphene walls at the tip, suggesting that closing of the tip happens simultaneously with the early stages of the etching process. It was also observed that the etching of the CCNT by atomic hydrogen proceeds via formation of an amorphous hydrocarbon layer as shown in Figure 6.6c. The subsequent removal of this amorphous layer eventually lead to straight tubes with only basal planes exposed, as shown in the inset of Figure 6.6 b. Earlier theoretical calculations demonstrated that graphene sheets can curl up with hydrogenation, which can justify the closing of the CCNT tips as observed during the diamond growth experiments ${ }^{85}$. On the other hand, there is no reasonable explanation for the observed selective nucleation of diamond crystals at the tips of conical carbon tubular structures. However, two possible explanations could be 1 . The caps generated during closing of the 
tip could serve as preferential sites for diamond nucleation due to increased hydrogen absorption at the curved surfaces ${ }^{119}$ and 2 . The aggressive etching of the wall surface could have prevented in formation of any nucleation site necessary for further growth of diamond crystal.
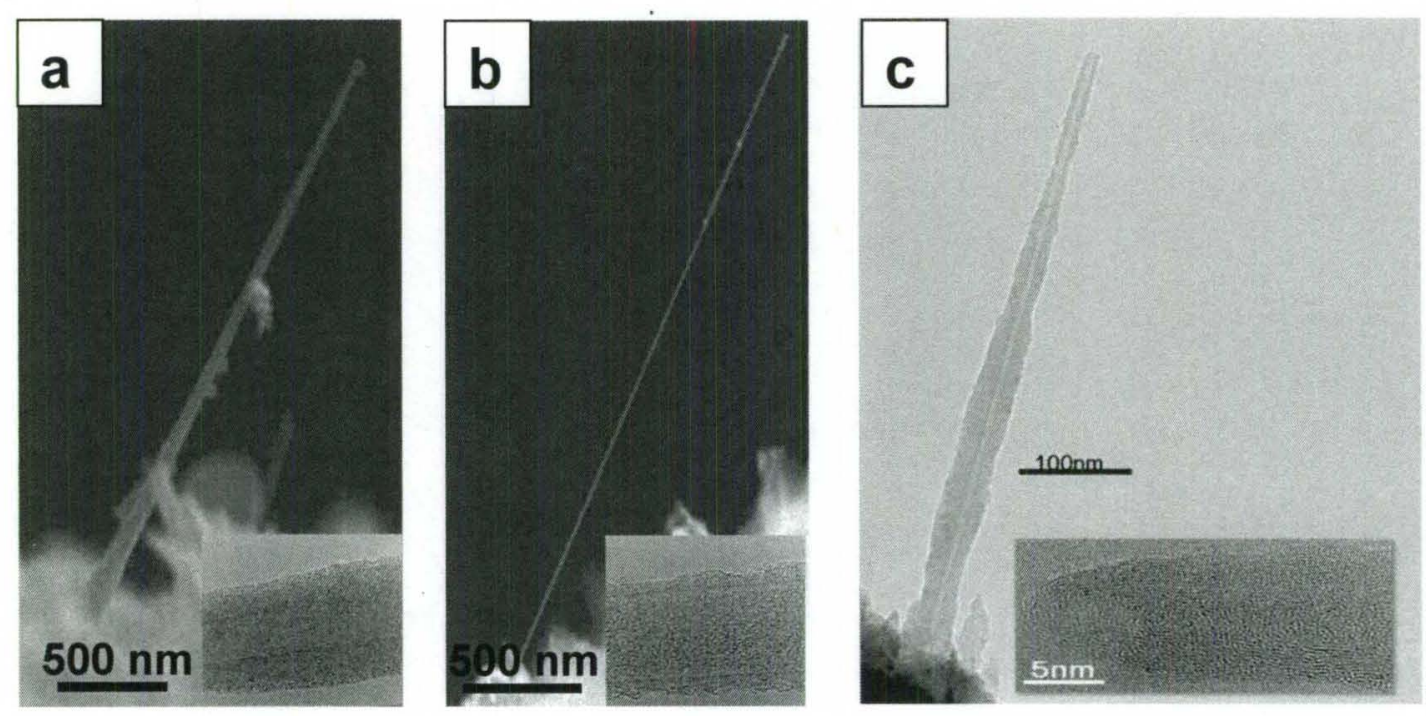

Figure 6.6 SEM image of an individual as synthesized CCNT (a) before etching, (b) after etching with insets showing the HRTEM image of the respective wall structures and (c) bright field image of a partially etched CCNT showing the partial etching of the wall structure with inset showing the HRTEM image of the closing of the tip.

\subsubsection{Carbon onions}

The above experiments suggested that higher atomic hydrogen concentrations present harsher and more aggressive etching conditions, thus preventing nucleation on the outer surface of the CCNT structures to form a nanocrystalline diamond coating. Experiments were conducted using electron cyclotron resonance (ECR) plasma under 50 
mTorr with CCNT samples which were pretreated by immersion in a sonicating diamond dispersion bath. Under these gentler plasma conditions, it was noticed that the etching of the CCNT walls was minimal, preserving the conical morphology, with small bright clusters on the CNP walls as shown in Figure 6.7a. Further examination of these structures using TEM revealed that the clusters were, in fact, carbon onion rings as shown in Figure $6.7 \mathrm{~b}$. The formation of carbon onions has been observed by other researchers during various processes such as electron-beam irradiation of carbon $\operatorname{soot}^{120}$, arcdischarge technique on carbon electrodes ${ }^{121}$ and a hot filament CVD (HFCVD) process with continuous flow of carbon powder ${ }^{122}$. The present observations of carbon onion formation on the sidewalls of conical carbon tubes with pretreatment suggest that the defects induced on the sidewalls of the CCNTs during pretreatment allow the formation of onions by curling of the walls in order to lower surface energy ${ }^{123,124}$. High resolution images of carbon onions, in Figure 6.7b, show the outward bending of the tube walls to form an onion structure, as illustrated in Figure 6.7c. Transformation of carbon onions to diamond by exposure to an electron or ion-beam irradiation was reported in a number of studies. The nanometer-scale carbon onion centers were shown to provide nucleation sites for diamond when irradiated with a high intensity electron beam ${ }^{125}$. The carbon onions were suggested to mediate the transformation of CNTs to diamond with laser irradiation ${ }^{123}$. In addition, using the CNTs as heaters and carbon onions as high pressure cells, in situ observations of quasimelting of diamond and diamond-graphite transformations were recently achieved ${ }^{126}$. It is not clear, though, how and whether the carbon onion structures observed in the present study could serve as nucleation centers for diamond with further exposure to atomic hydrogen under CVD conditions. 

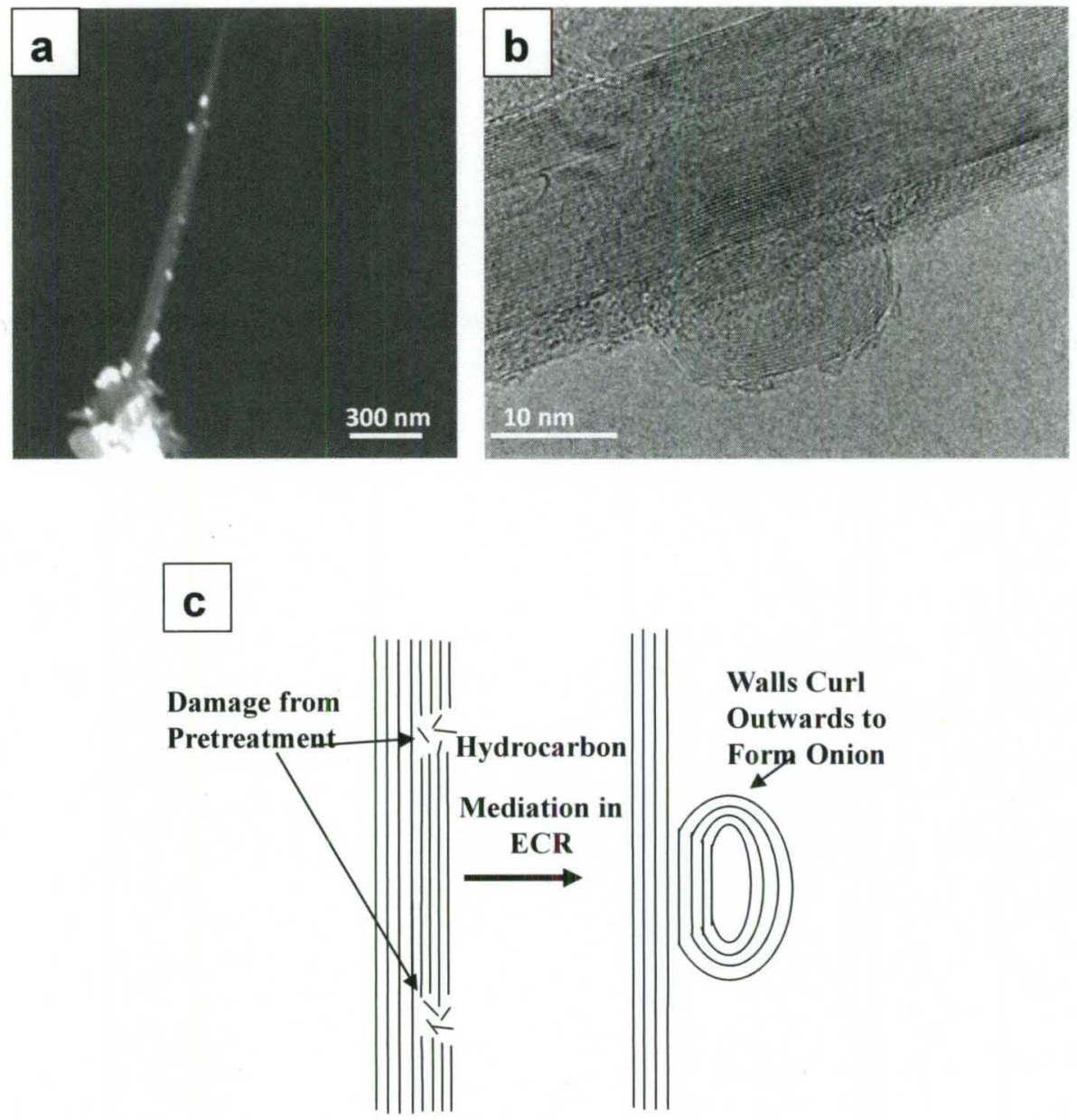

Figure 6.7 (a) SEM image showing several bright clusters on the sidewall of CCNT, (b) HRTEM image of the one of the bight clusters exhibiting the carbon onion structure and (c) a schematic illustration of mechanism of the curling of CCNT walls at the damage sites created during the pretreatment step.

\subsection{Nucleation and growth mechanism}

It was observed that the most interesting aspect of pretreatments was that both sonication and electrophoretic seeding significantly influenced the resulting diamond 
nucleation and growth on the wall structures of the CCNTs. The reasons are not obvious, so the early stages of diamond nucleation on the walls of CCNT structures were studied using HRTEM. Figures $6.8 \mathrm{a}$ and $6.8 \mathrm{~b}$ show the originating of the diamond crystal within the inner tube walls of the CCNT. The presence of both diamond and multiwalled graphite is supported by the indexed fast Fourier transform (FFT) pattern of the HRTEM image shown in the inset of Figure $6.8 \mathrm{~b}$. The forbidden (020) reflection is seen in the FFT pattern in Figure $6.8 \mathrm{~b}$. This may be due to kinematic effects in crystals with thicknesses greater than $10 \mathrm{~nm}$, or in a crystal that has fcc or "n-diamond" phase ${ }^{93}$. As the crystal seen here is about $10 \mathrm{~nm}$ or larger, it is probably due to thickness effect rather than the crystal being fcc carbon phase. The FFT reconstruction from the diffraction maxima corresponding to a diamond-cubic structure is shown in Figure 6.8c. The FFT reconstruction from the diffraction bands corresponding to multiple graphene walls is shown in Figure 6.8d. These results confirm that the core diamond crystal is surrounded by multi graphene walls. The energy loss near edge structure (ELNES) in Figure 6.8e for carbon-K presents $\pi^{*}$ and $\sigma^{*}$ edges. Generally, the ELNES of C-K edge in diamond has $\sigma^{*}$ peaks, where as for graphene there is only a $\pi^{*}$ peak $^{90,127}$. In our spectrum of the edge of nanodiamond crystal, both $\pi^{*}$ and sharp $\sigma^{*}$ peaks are evident, thus showing that the nanodimond crystallines on the CNP walls are surrounded by multi-graphene walls. 

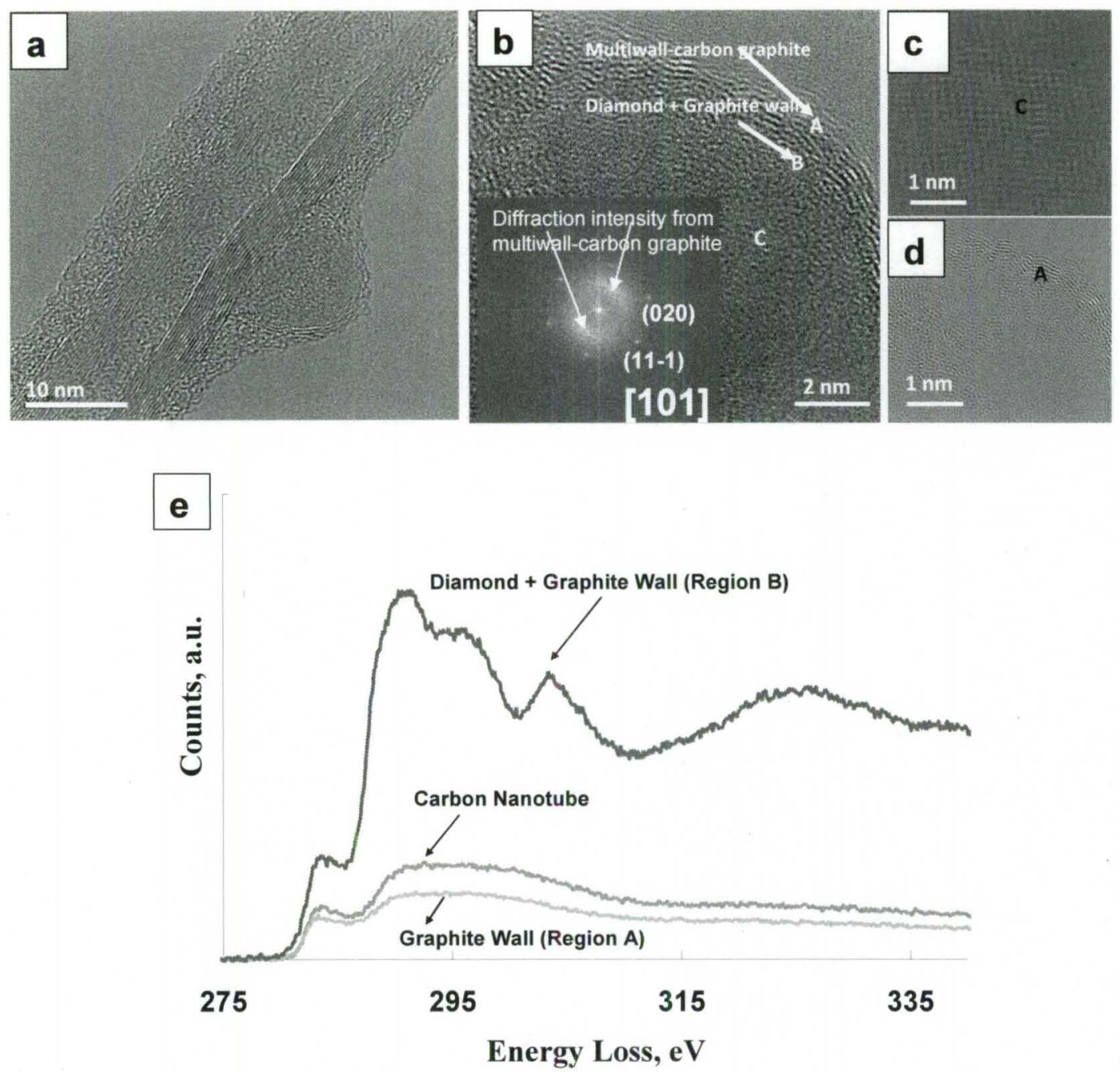

Figure 6.8 (a) HTREM image of a diamond crystal nucleated within the subsurface layer, (b) A subsurface diamond crystal is shown with regions labeled as ' $A$ ' for tube walls, ' $B$ ' for the region between diamond and tube wall, and ' $\mathrm{C}$ ' for the inner diamond crystal. The inset is an FFT pattern showing the diffraction bands and maxima resulting from both multi-walled carbon tube and diamond, respectively, (c) FFT reconstructed image from the maxima corresponding to diamond from region ' $\mathrm{C}$ ' and (d) FFT reconstructed image from the diffraction corresponding to the multiwall graphite from region ' $A$ '. (e) ELNES of carbon-K edge showing a mixture of diamond and multi-walled carbon tube. 
In order to study the role of type of the seeding particle on the nucleation, experiments involving the pretreatment of CCNT with boron carbide powder $(0-7 \mathrm{~mm})$ were also conducted. The results are similar to those treated using diamond powder, i.e., simple dipping into ultrasonic bath containing boron carbide powder resulted in an increased density of crystals at the CCNT tips and electrophoretic seeding resulted in a high density of nucleation along the sidewalls, as shown in Figure 6.9. This is a significant result showing that nucleation onto the carbon tubular structures can be enhanced by pretreatments utilizing powders other than diamond, and the pretreatment powders do not act like seeds.
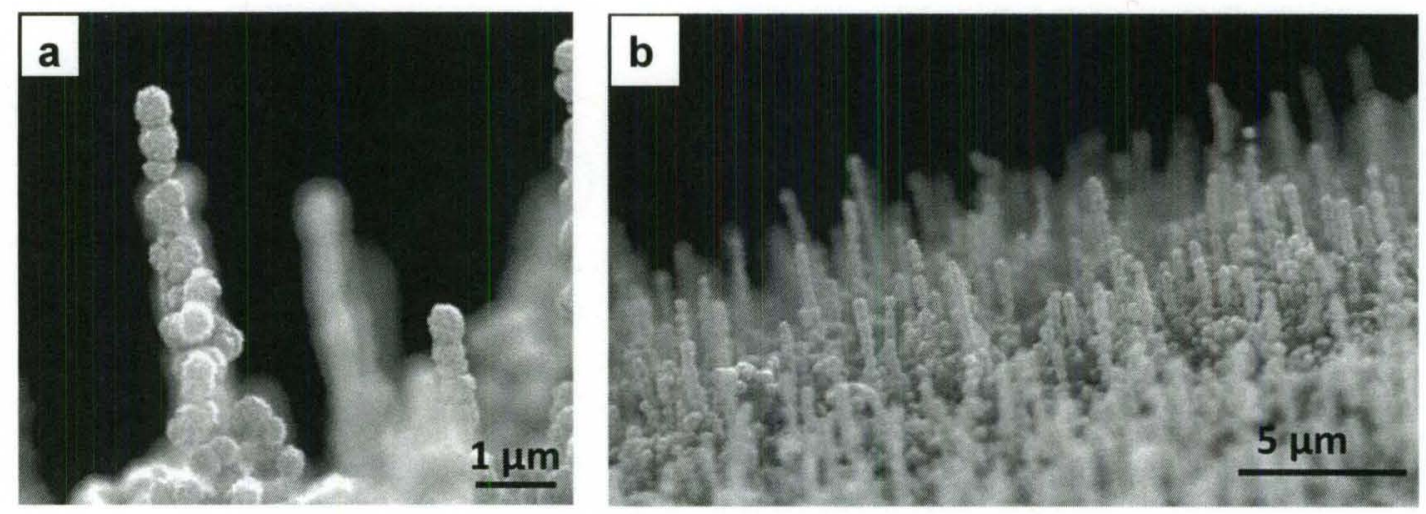

Figure 6.9 SEM images showing CNPs treated with boron carbide powder (a) Shows the complete coating of diamond crystals along the length of CNP clearly depicting the spiral growth of diamond crystals and (b) shows large array of CNPs coated with diamond crystals.

Based on all the above observations, a possible mechanism is proposed for the nucleation of diamond with pretreatment. 1) The impacts by seed particles during pretreatment damage the wall structure of the CCNTs introducing subsurface defects. 2) 
Hydrogenation of the subsurface defects forms an amorphous hydrogenated carbon phase. 3) Subsequent slow hydrogenation of the amorphous carbon phase regions leads to the nucleation of diamond, whereas nucleation on the outer walls is prevented due to the aggressive etching conditions. It had been previously reported that defects on CNT walls serve as sites for diamond nucleation through formation of an amorphous carbon phase ${ }^{92}$. Diamond nucleation through transition from amorphous carbon has also been described on noncarbon substrates ${ }^{89,90}$.

\subsection{Summary}

In this chapter, nucleation of the diamond on CCNTs has been presented. It was shown that, diamond nucleated selectively at the tips of CCNTs with no pretreatment. However, with pretreatments using either diamond or boron carbide powder dispersions improved the nucleation density on the tips and also on the sidewalls, forming a nanocrystalline diamond coating on the CCNTs. A likely mechanism of slow hydrogenation kinetics at subsurface defects created during pretreatments leading to diamond nuclei formation inside the walls of MWNTs or CCNT structures was also proposed. Low pressure hydrogen plasma experiments in ECR resulted in the nucleation of carbon onions on the sidewalls of CCNTs. 


\section{CHAPTER 7}

\section{THERMIONIC EMISSION FROM PHOSPHORUS DOPED DIAMOND \\ CRYSTALS}

\subsection{Introduction}

Diamond grown using the chemical vapor deposition method exhibits negative electron affinity (NEA) - i.e., the presence of a vacuum level below the conduction band minimum $(\chi=-1.27 \mathrm{eV})$ due to hydrogen termination on the surface, thus reducing the work function ${ }^{13}$. In addition, n-type doping into diamond has been shown to introduce shallow donor levels close to conduction band minimum which also can lower the work function to a greater extent. Thus, diamond with reduced work function values along with its highest thermal conductivity and thermal and electrical stability can be expected to be a promising candidate for thermionic emission energy conversion.

n-type doping in diamond has been achieved using several dopants such as nitrogen, sulfur, and phosphorus and also by co-doping with different dopants ${ }^{14,39,44}$. However, only phosphorus has been reported to introduce shallow donor levels very close to the conduction band minimum $(0.6 \mathrm{eV})^{44}$. However, phosphorus incorporation in diamond has been challenging due to its larger atomic radius. In addition, most of the earlier work involved the study of doping and thermionic emission characteristics from either diamond films or single crystal diamond, preventing the study of a true diamond 
nano crystal free from grain boundaries. Thus, in this chapter, we study phosporous doping into individual diamond nanocrystals using special hybrid architecture which can be expected to exhibit enhanced thermionic emission properties combining the advantage of both carbon and diamond nano structures. Phosphorous doping in diamond films is also studied using UPS measurements.

\subsection{Results}

\subsubsection{Thermionic emission from diamond crystals on CCNTS}

In order to study the effect of phosphorous doping of diamond crystals on thermionic emission characteristics, three different types of samples were synthesized. These include as-synthesized CCNTs, un-doped diamond crystals on CCNTs, and phosphorous doped diamond crystals on CCNTs. Figure 7.1 shows the SEM image of the individual CCNTs of each of these samples on graphite foil substrates. The experimental conditions employed were same as those described earlier. As synthesized CCNT arrays similar to the ones shown in Figure 7.1a were used as substrates for growing both undoped and phosphorous doped diamond crystals. The size of the undoped and doped diamond crystals is in the range of $1-2 \mu \mathrm{m}$. 

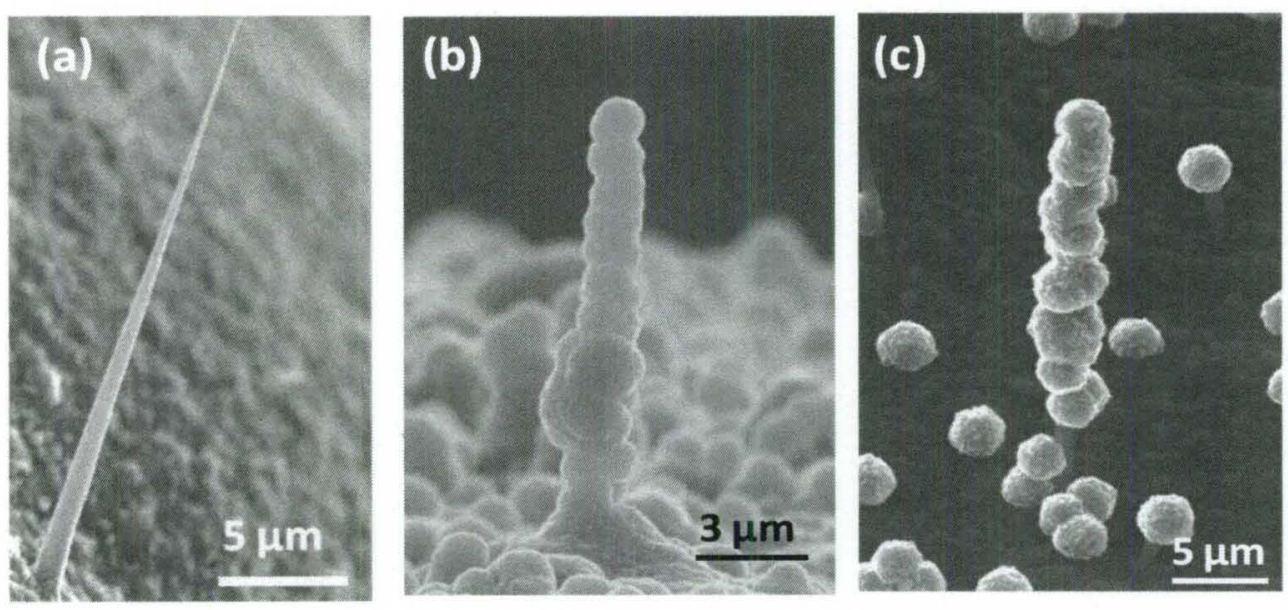

Figure 7.1 SEM images of individual (a) as synthesized CCNT, (b) undoped diamond coated CCNT and (c) Phosphorous doped diamond crystals coated CCNT on graphite foil substrates.

The presence of the phosphorus in the diamond crystals of sample 3 was confirmed from the secondary ion mass spectroscopy (SIMS), but quantitative value cannot be reported owing to rough analytical surface (as shown in Figure 7.2). 


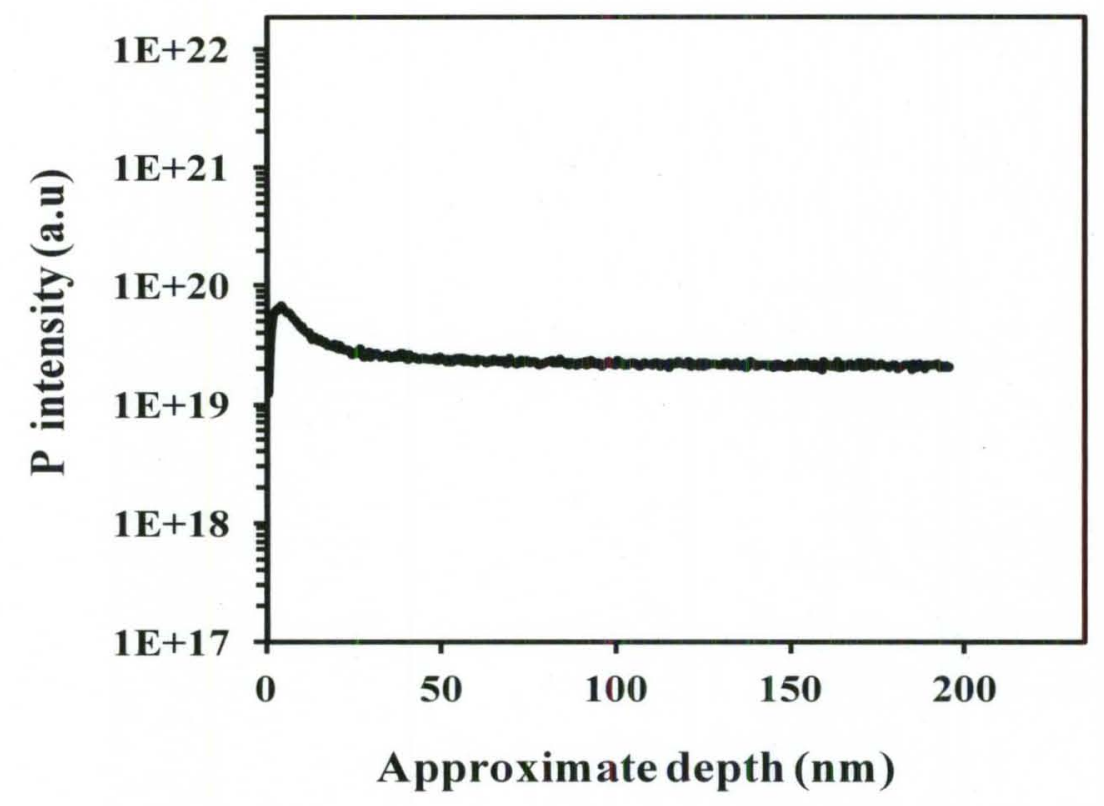

Figure 7.2 Secondary ion mass spectroscopy (SIMS) depth profile of phosphorus in diamond crystals grown on CCNTs

The resistivity of the substrate can be essentially ruled out in the present case due to the presence of electrically conducting substrate (graphite foil) and underlining conducting structures (CCNTs) for these diamond emitters. This is advantageous for the thermionic emission applications as reduced emitter resistivity can result in significant increase in the emission current density ${ }^{53}$. The work function values of each of the sample can be determined from the following thermionic emission characteristics. The work function was calculated using Richard-Dushman formula ${ }^{6}$

$$
J_{0}=A T^{2} e^{-\left(\frac{\Phi}{k T}\right)}
$$

which describes the dependence of the zero field current density $J_{0}$ on temperature $T$. $\Phi$ is the work function of given material, $k$ is Boltzmann constant. Constant $A$ can be 
expressed by equation $A=\lambda_{B} \cdot \lambda_{R} \cdot A_{0}{ }^{128}$. Where $A_{0}$ is Richardson constant; $\lambda_{R}$ and $\lambda_{B}$ are correction factors specific for particular material. Thus value of constant $A$ could significantly vary from the value of Richardson constant $A_{0}{ }^{129}$.

Zero field thermionic emission current density $J_{0}$ could be calculated from formula describing Schottky effect ${ }^{6}$

$$
\begin{gathered}
J=J_{0} e^{-\left(\frac{C \sqrt{E}}{k T}\right)} \\
C=\sqrt{\frac{e^{3}}{4 \pi \varepsilon_{0}}}
\end{gathered}
$$

Where $e$ is the electric charge, $\varepsilon_{0}$ is a dielectric constant, $E$ is the external electric field on cathode (sample's) surface and can be expressed as $E=\alpha U$, where $U$ is the voltage drop between anode and cathode and $\alpha$ is the constant dependent on geometry of the cathode structure $^{27}$. Thus from thermionic $I-U$ characteristic and $L n I$ vs $\sqrt{U}$ curves (Figure 7.3 (a) and (b)), $J_{0}$ values at different temperatures were determined (intercept values of $\operatorname{LnI}$ vs $\sqrt{U}$ curves) ${ }^{6}$. 

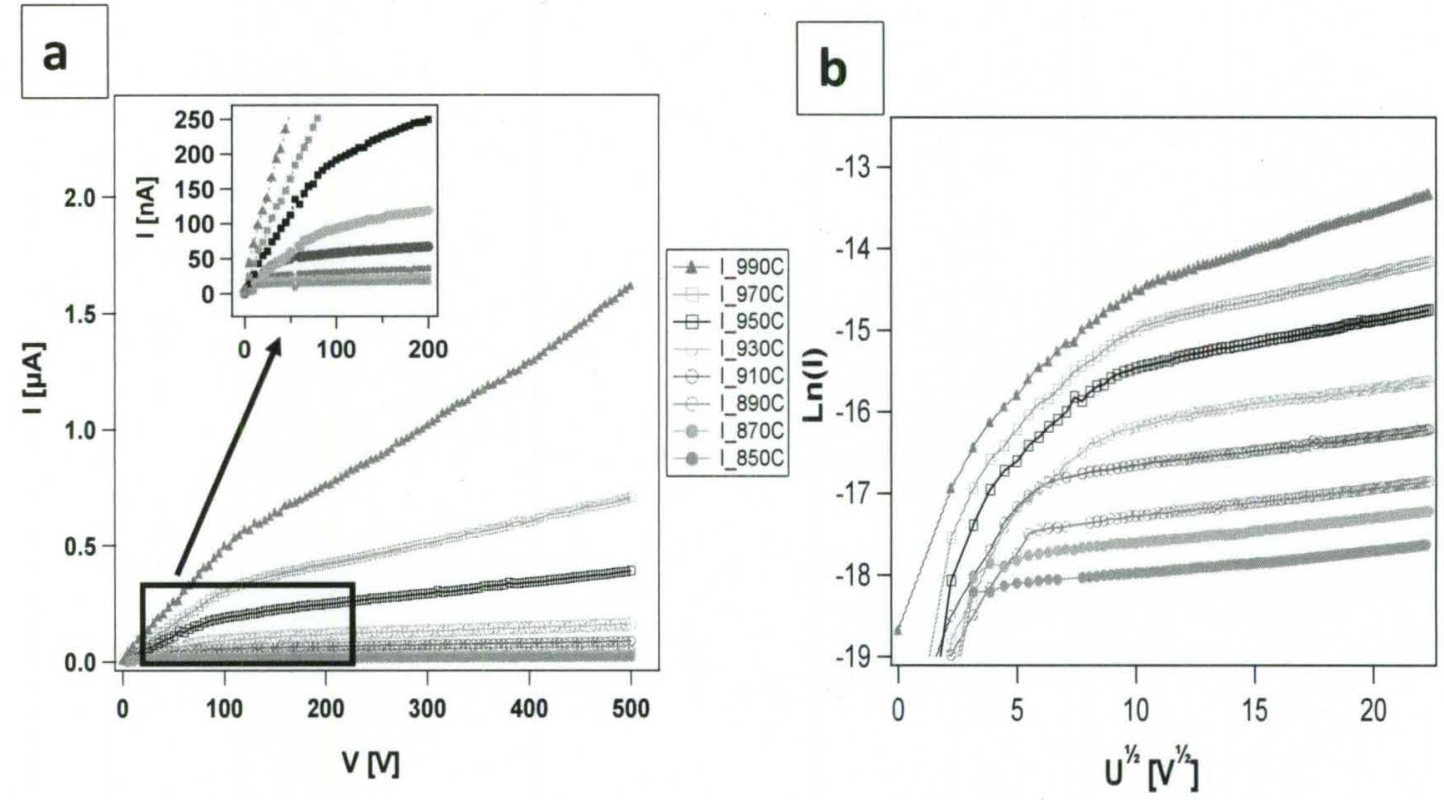

Figure 7.3 Thermionic emission measurements plots of $\mathrm{P}$ doped diamond coated CCNTs (a) showing thermionic $\mathrm{I}-\mathrm{V}$ characteristics, (b) showing $\mathrm{Ln}(\mathrm{I})$ vs $\mathrm{U}^{1 / 2}$ curves for various temperatures in ${ }^{\circ} \mathrm{C}$.

Using Richardson equation and by fitting experimental curves $\operatorname{Ln}\left(\frac{J_{0}}{T^{2}}\right) \operatorname{vs} \frac{1}{T}$, the values of work function for each sample can be obtained as shown in Figure 7.4a. The work function values obtained for bare CCNT, undoped and phosphorous doped diamond coated CCNT are $4.01 \mathrm{eV}, 4.26 \mathrm{eV}$ and $2.2 \mathrm{eV}$, respectively. As shown in Figure $7.4 \mathrm{~b}$ the temperature $\left(1053^{\circ} \mathrm{K}\right)$ at which emission started in case of phosphorous doped diamond is lower than the other two samples, which could be attributed to its lower work function value. 

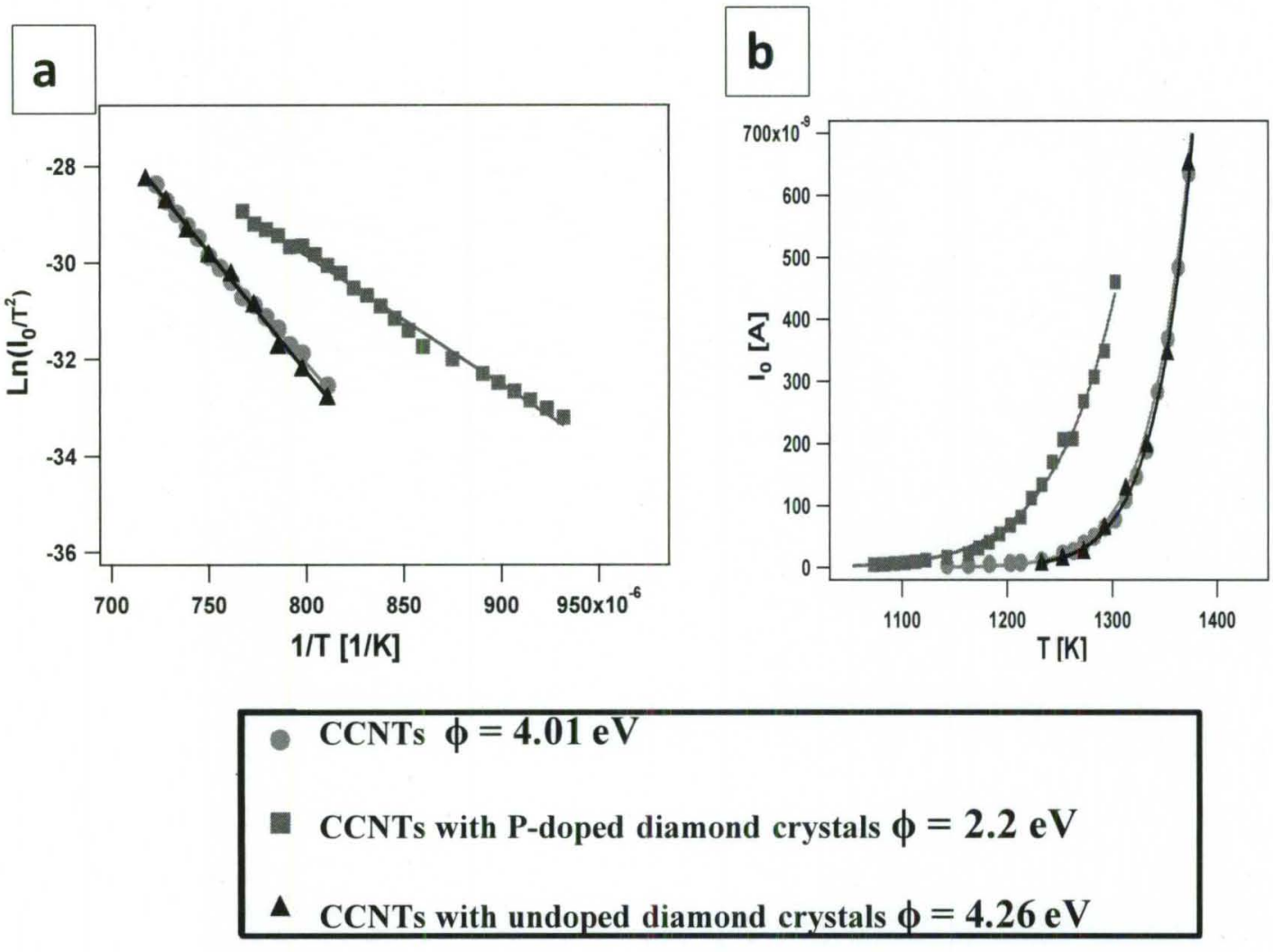

Figure 7.4 Thermionic emission data (dots) and data-fit (line) to Richardson-Dushman equation from as synthesized CCNTs, CCNT coated with undoped diamond and CCNT coated with phosphorus doped diamond

\subsubsection{UPS studies on undoped and phosphorous doped diamond films}

The phosphorus doped diamond films on silicon substrates were synthesized as described in the experimental section of chapter 3. Work function measurements of phosphorous doped diamond films were carried out using UPS, the experimental set up of which was also described earlier. The samples for the measurements had the active material in electrical contact with gold film sputtered on copper foil to equilibrate the Fermi levels $\left(\mathrm{E}_{\mathrm{F}}\right)$. A part of the copper foil sputtered with gold was used for placing the sample and the other half for acquiring the reference gold spectra. Double side copper 
tape was used for the electrical contact between silicon substrate with diamond film and the underlining gold film on copper foil. The measurements were performed using He-I $(21.23 \mathrm{eV})$ and He-II $(40.81 \mathrm{eV}) \mathrm{UV}$ excitations. A stable bias was provided to avoid the instrumental cut-off in the lens system of the analyzer at low-kinetic energy (KE) for all the UPS spectra measurements. The external bias that was measured and the spectra were shifted back to zero-bias position through post acquisition data processing. The calibration of the UPS spectrometer was performed by measuring the absolute position of the Fermi level of the gold sample using He-I. A spectra, measured using a negative bias of $4.9 \mathrm{eV}$ is shown in the Figure 7.5a resulting in $\mathrm{E}_{\mathrm{F}}$ value of $21.2 \mathrm{eV}$ that is in well agreement with the expected value. Similarly He-II spectrum (Figure 7.5b) shows Fermi level value of $40.83 \mathrm{eV}$, which also agrees with the literature value ${ }^{130}$. A work function value of $4.67 \mathrm{eV}$ is obtained for gold film that matches the value range reported in the literature ${ }^{131}$.
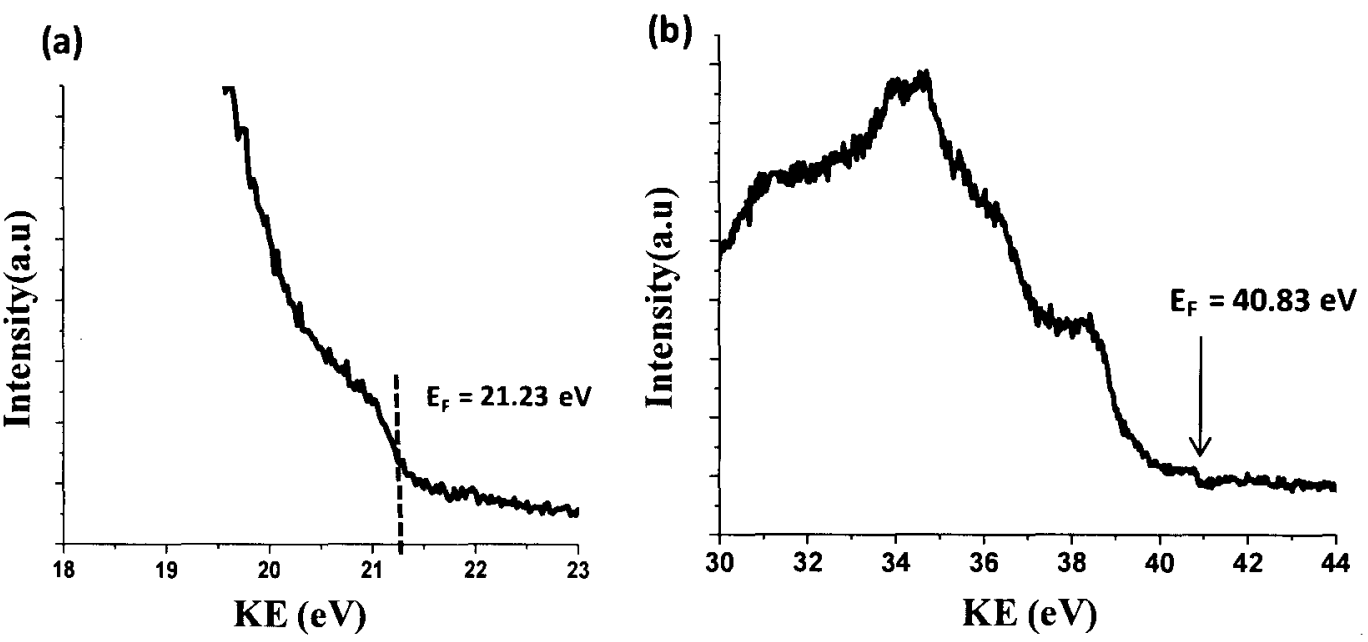

Figure 7.5 UPS spectra of glod film showing Fermi level positions at (a) $21.23 \mathrm{eV}$ for $\mathrm{He}-\mathrm{I}$ (b) $40.83 \mathrm{eV}$ for He-II radiations 
Figure 7.6 shows the low kinetic energy part of the He-I emission spectra of a) undoped and b) phosphorus doped diamond films. Fermi level of the spectra is assumed to be aligned to that of gold Fermi as the diamond films are in good electrical contact with underlining gold film. The work function of the material is then determined from the intersection of low-KE cut off tail with the background level ${ }^{132}$. All the numbers for the cut-off energy positions are obtained with the extrapolation to zero intensity. Both undoped and phosphorous doped diamond films exhibit two peak behavior one at low KE cut-off and other a high intensity peak characteristic of work function and NEA behavior with electrons coming from CBM respectively ${ }^{133}$. In the case of undoped diamond, the value of the work function was determined as $3.9 \mathrm{eV}\left(\mathrm{E}_{\mathrm{F}}-\mathrm{E}_{\mathrm{Vac}}\right)$ and the $\mathrm{CBM}$ position at $5.1 \mathrm{eV}\left(\mathrm{E}_{\mathrm{F}}-\mathrm{E}_{\mathrm{C}}\right)$, which gives a NEA value of $1.2 \mathrm{eV}$. Similarly for phosphorous doped diamond, a work function value (low KE cut-off) of $1.8 \mathrm{eV}$ and NEA value of $1.2 \mathrm{eV}$ was obtained. 
(a)

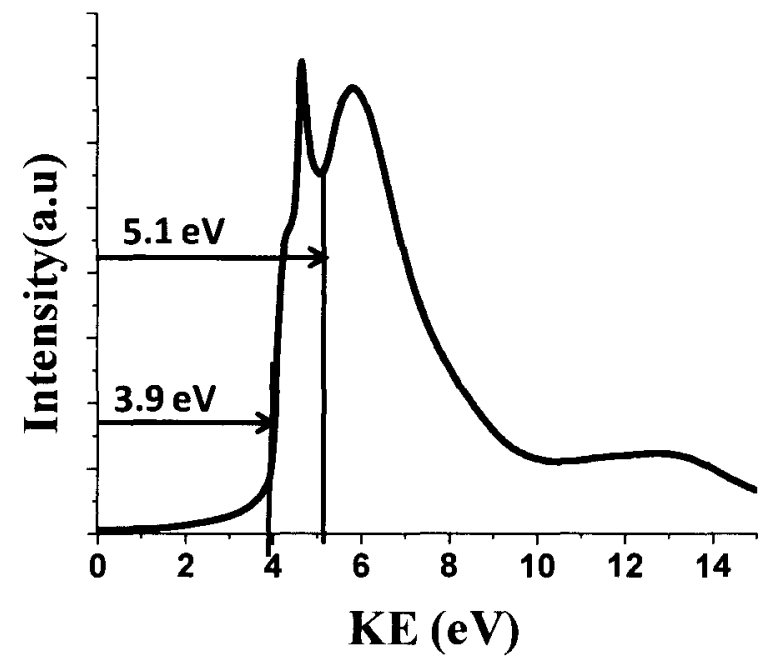

(b)

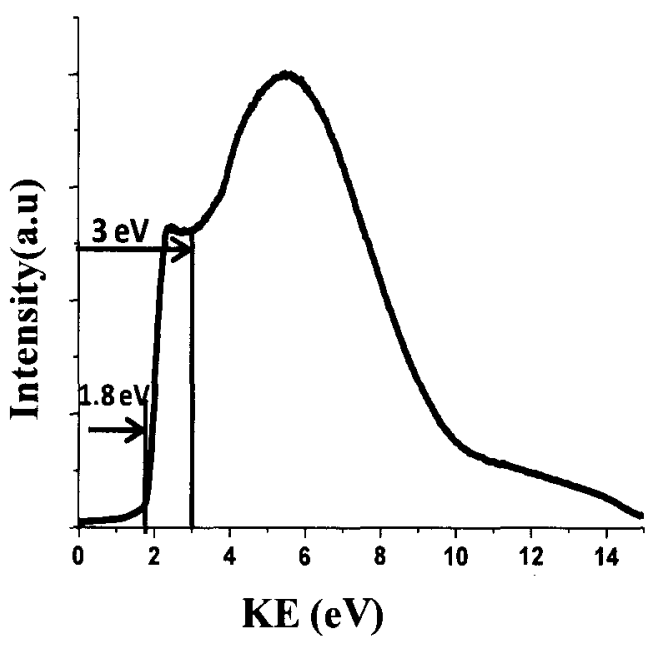

Figure 7.6 UPS photoemission spectra (low kinetic-energy $\mathrm{He}-\mathrm{I}(21.23 \mathrm{eV})$ ) of a) undoped diamond films, b) phosphorus doped diamond film showing low kinetic cut-off energy positions indicative of work function and a high intensity cut-off position of conduction band minimum due to the presence of NEA

Commonly accepted value of NEA for (111) diamond surface was reported as less than $1.2 \mathrm{eV}^{134}$ and nearly equal to $1.3 \mathrm{eV}$ for (100) diamond surface ${ }^{135}$. The observed value of NEA of $1.2 \mathrm{eV}$ in the present case is in well agreement with the value of NEA of less than $1.1 \mathrm{eV}$ for a phosphorus doped diamond (111) film ${ }^{136}$. Though, the presence of NEA was also seen in the case of undoped diamond film, the observed lower work function value of $1.8 \mathrm{eV}$ in the case of phosphorus doped diamond film can be attributed to additional possible donor states introduced by phosphorus along with NEA. The observed work function value of $3.9 \mathrm{eV}$ for undoped diamond film is in well agreement with other earlier reports ${ }^{137}$. 
Recently a work function value of $3.6 \mathrm{eV}$ was reported for hydrogen terminated phosphorus doped (111) single crystal diamond ${ }^{138}$ using photoelectron spectroscopy. An upward band bending of $3.2 \mathrm{eV}$ from the simulated surface Fermi level position and absence of NEA from the UPS spectra was attributed to the large work function value. Though the presence of the NEA was predicted theoretically, the reason for the absence could not be evidently articulated. However, in the present case the presence of NEA value of $1.2 \mathrm{eV}$ evident from the UPS spectra and the presence of phosphorus doping into diamond films evident from SIMS plot (Figure 7.7) could be ascribed to the observed reduced work function value of $1.8 \mathrm{eV}$. However, further theoretical calculations and experimental studies might be needed to accommodate for the all the differences observed in the present scenario for the determination of the surface Fermi level position and band bending values.

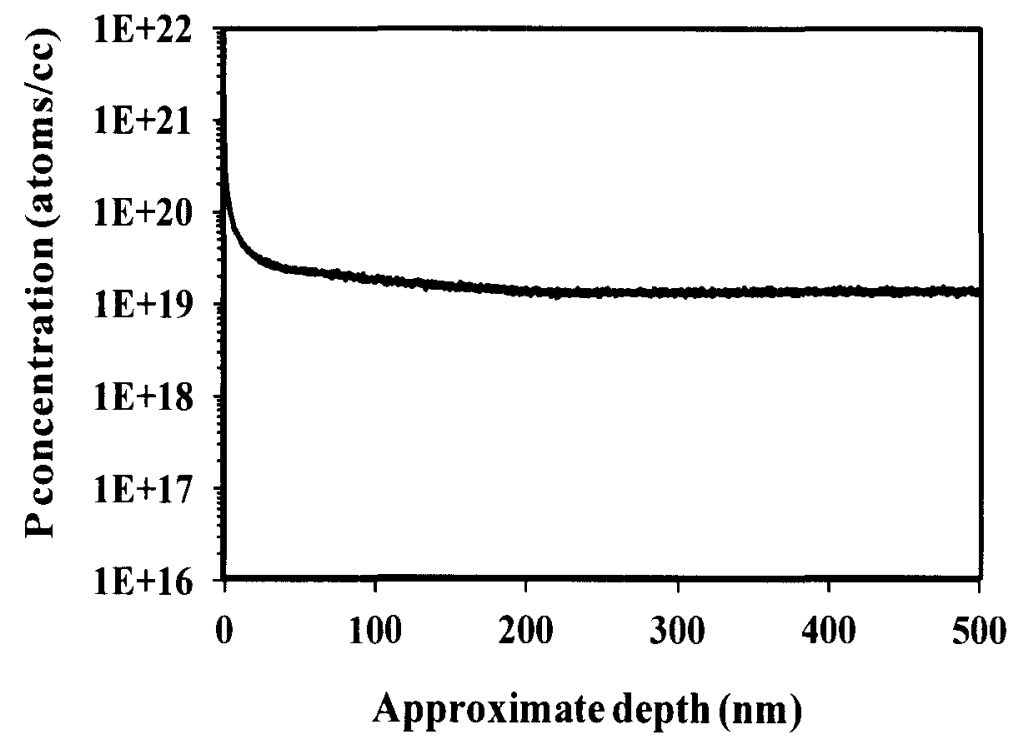

Figure 7.7 Secondary ion mass spectroscopy (SIMS) depth profile of phosphorus in Pdoped diamond films on silicon wafer 


\subsubsection{Phosphorus doped, individual diamond crystals}

From thermionic emission results (Figure 7.5), it is very evident that the phosphorus doped diamond sample has lower work function value compared to bare and undoped diamond coated CCNT arrays. This can be attributed to the raising of the Fermi level closer to the conduction band due to the presence of donor states introduced by phosphorus. Both the donor levels and NEA play an important role in lowering the work function value by changing the Fermi and vacuum levels respectively. However, in this case, the role of NEA in observed work function value can be assumed to be negligible, because the work function values were measured using thermionic emission at temperatures greater than $1000{ }^{\circ} \mathrm{C}$. The loss of NEA from diamond at such high temperatures was previously reported by several groups ${ }^{50,139}$. The detected work function value as low as $2.2 \mathrm{eV}$ in the case of diamond crystals with negligible NEA can be attributed to possible higher incorporation of dopants in individual diamond micron size crystals compared to diamond films for a given doping concentration. Because, the absence of same NEA in the case of phosphorous doped diamond films would result in higher work function value of about $3.6 \mathrm{eV}$ with a possible positive electron affinity of $0.4 \mathrm{eV}^{134}$, which is in close approximation with reported value ${ }^{138}$. Thus individual diamond crystals free from grain boundaries and with reduced size effects can result in enhanced thermionic emission characteristics due to formation of shallow donor levels compared to diamond films. In addition, the saturation emission current density obtained from these phosphorus doped diamond crystals (average diameter of $1.6 \mu \mathrm{m}$ and number density of $5.6 \times 10^{4} / \mathrm{cm}^{2}$ ) at $900{ }^{\circ} \mathrm{C}$ (Figure 7.3) is approximately equal to $0.2 \mathrm{~mA} / \mathrm{cm}^{2}$, which is similar to the order of magnitude of previous report from nitrogen doped 
diamond nanocrystalline film ${ }^{51}$. The saturation emission current density of these crystals was calculated considering the average surface area of the diamond crystals at the tips of CCNTs (determined from SEM images) as the actual emission area. Additional increase in the density of these diamond crystals can result in significant increase in emission current density owing to geometrical increase of emission surface area. Further studies of these nanocrystals at atomic scale would be required for determining the accurate doping concentration and band structure.

\subsection{Summary}

In this chapter, successful phosphorus doping into diamond microcrystals using hybrid architecture consisting of CCNTs is described. Work function value as low as 2.2 $\mathrm{eV}$ was achieved from these phosphorous doped crystals with absence of NEA from thermionic emission characteristics. Phosphorus doping into diamond films has also been demonstrated and Ultraviolet photospectroscopy (UPS) studies from these films revealed work function values of $1.8 \mathrm{eV}$ with presence of $\mathrm{NEA}$ value of $1.2 \mathrm{eV}$. Possible presence of shallower donor levels in diamond micro crystals compared to diamond films for a given concentration of phosphorous doping can be attributed to higher incorporation of phosphorus in smaller diamond crystals. 


\section{CHAPTER 8}

\section{CONCLUSIONS}

In this dissertation, a new hybrid architecture consisting of phosphorus doped diamond crystals and carbon nanostructures was discovered for thermionic emission energy conversion applications. The synthesis and study of this novel structure was carried out broadly in three major steps. The growth of the phosphorus doped individual diamond crystals could not be achieved without appropriate template, for which CCNTs was chosen in the present case due to their special characteristic features. Thus in the first step, nucleation and growth of CCNTs was studied for their synthesis on flat substrates in large area. The field and thermionic emission characteristics of as synthesized CCNTs were studied not only due to their superior characteristic features, but also for the understanding of the emission properties of the hybrid architecture. In the second step, nucleation of diamond nanocrystals on CCNTs was studied for developing the hybrid architecture. Finally phosphorus doping into these diamond crystals on CCNTs was carried out and thermionic emission characteristics were measured. Doping into diamond films was also achieved and their work function values were determined using UPS.

\subsection{Large area synthesis of CCNTs}

- $\quad$ CCNTs with superior aspect ratios and uniformity were obtained in large areas $\left(\sim 1 \mathrm{~cm}^{2}\right)$ on planar graphite foil substrates. 
- The catalyst particle was shown to facilitate the nucleation of CCNTs only in the initial stages from the absence of the catalyst at the tip or the base of these structures.

- The evolution of the conical morphology was attributed to the difference in the rates of vertical growth by attachment of carbon atoms at edges of growing graphene sheets and radial growth with epitaxial nucleation of new graphene layers at the bottom with no catalyst.

- $\quad$ Regrowth experiments on straight MWNTs supported the proposed mechanism for the growth of conical structures.

- Growth kinetics data revealed that the growth rate is linearly proportional to the ratio of diameter to length $(d / l)$ of the $\mathrm{CCNT}$ validating the kinetic impingement limited growth model for CCNTs.

- Higher synthesis pressures resulted in the growth of nanohorns, rings, polyhedral shells and graphene super lattice structures.

\subsubsection{Moire patterns of bilayer graphene}

- $\quad$ Adjacent Moire patterns consisting of a triangular superlattice and parallel straight bands in few layer graphene was observed using TEM

- A pictorial model for the observed Moire pattern for the few layer graphene superlattice was presented and shown that parallel bands are a consequence of change in shape of a part of graphene layer from rectangular to parallelogram.

\subsubsection{Field and thermionic emission characteristics of CCNT}

- Different CCNTs samples with varying different densities, radii of curvatures, lengths and wall structures were synthesized by varying the critical growth parameters. 
- Field emission studies from these conical structures resulted in a turn-on electric field as low as $0.7 \mathrm{~V} / \mu \mathrm{m}$ and field enhancement factor as high as 7,600.

- It was shown that an optimum emitter density of CCNTs plays an important role in reducing the field screening effects for enhanced the field emission properties along with their superior characteristic features.

- $\quad$ Thermionic emission measurements from CCNT arrays resulted in observed work function value of about $4 \mathrm{eV}$ which is lower than that of pristine CNTs.

- Work function values of CCNTs on platinum and graphite foil substrates were also confirmed using Ultraviolet photospectroscopy studies.

\subsection{Nucleation and growth of diamond nanocrystals on CCNTs}

- A selective nucleation of diamond crystals only at the tips of CCNTs or complete coating of CCNTs with diamond nanocrystals was achieved varying growth and pretreatment procedures.

- A thermal run away scenario of excess heat buildup on the diamond crystal at the tip of CCNT was attributed to the observed rough morphology of the diamond crystal.

- A likely mechanism of slow hydrogenation kinetics at subsurface defects created during pretreatments leading to diamond nuclei formation inside the walls of MWNTs or conical CNT structures was also proposed.

- Low pressure hydrogen plasma experiments in ECR resulted in the nucleation of carbon onions on the sidewalls of CCNTs.

\subsection{Thermionic emission from phosphorus doped diamond crystals}


- Phosphorus doping into diamond crystals on CCNTs was achieved employing a liquid Di-tert-butyl phosphine using bubbler set up.

- Work function value of $2.2 \mathrm{eV}$ was achieved from these P-doped diamond micro crystals from thermionic electron emission characteristics.

- Ultraviolet photospectroscopy (UPS) studies from phosphorus doping into diamond films revealed work function values of $1.8 \mathrm{eV}$ with the presence of NEA of 1.2 eV.

- Possible presence of shallower donor levels in diamond micro crystals compared to diamond films for a given concentration of P-doping can be attributed to higher incorporation of phosphorus in smaller diamond crystals. 


\section{CHAPTER 9}

\section{FUTURE SCOPE}

The study of individual diamond nano rystals isolated from the underlining CCNT structures in the hybrid architecture could provide deeper insight for understanding the doping and band structure aspects of it towards various applications. In the case of bulk materials or epitaxial films, techniques such as SIMS (secondary ion mass spectroscopy), hall measurements and capacitance-voltage profiles can be used to measure atomic concentrations of dopants and carrier concentrations. However, these traditional techniques could not be applied in the present case due to small dimentions and $3 \mathrm{D}$ morphologies of these nanostructures. Atom probe tomography (APT) is the only material analysis technique that can offer extensive capabilities for both $3 \mathrm{D}$ imaging and chemical composition measurements at the atomic scale (around 0.1-0.3nm resolution in depth and $0.3-0.5 \mathrm{~nm}$ laterally). Thus accurate doping concentration in the individual diamond crystals can be measured for understanding of the electronic properties of these nanocrystals.

\subsection{Atom probe tomography (APT)}

In APT, atoms at the specimen surface are field evaporated using high DC voltage $(5-20 \mathrm{kV})$. The schematic illustration of the experimental set up is shown in the Figure 9.1. The sample prepared in the form of a very sharp tip and the high voltages induce a 
very high electrostatic field (tens $\mathrm{V} / \mathrm{nm}$ ) at the tip surface, just below the point of atom evaporation. Under laser or HV pulsing, a few atoms are evaporated from the surface, by field effect (near 100\% ionization), and projected onto a position sensitive detector (PSD).

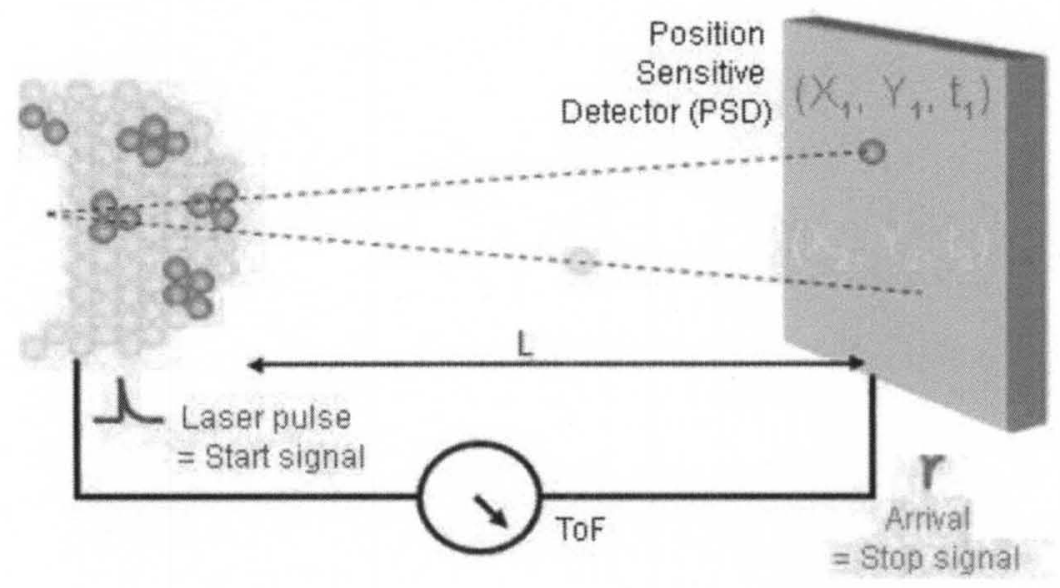

Figure 9.1 Schematic illustration of atom probe tomography experimental set up

The detector allows simultaneous measurement of the Time of Flight of the ions i.e. measuring the time between the laser flash and the arrival on the PSD allows to determine the $\mathrm{m} / \mathrm{q}$ ratio (mass over charge ratio) and the $(\mathrm{X}, \mathrm{Y})$ position of the ion impact on the detector: measuring the X-Y position and the order of arrival of the ions on the PSD allows to reconstruct the original position of the atoms on the tip. By repeating this sequence, the atoms are progressively removed from the tip, and a $3 \mathrm{D}$ image of the material can be reconstructed at the atomic scale.

\subsection{Nitrogen and phosphorus doping into diamond nanocrystals and CCNTs}

The size of the diamond crystals on the CCNTs was lowered to about $100 \mathrm{~nm}$ using special pretreatment techniques. Nitrogen and phosphorus doping into these 
crystals will be attempted for studying the field and thermionic emission characteristics. Doping into CCNTs with exposed graphitic edge planes can be expected to yield fundamentally fascinating scientific discoveries and their emission characteristics can be explored.

\subsection{Electrochemical studies of phosphorus doped diamond crystals and films}

Diamond is a prospective electrode for electrochemical applications due to its wide potential window and low back ground currents. The electrochemical behavior of diamond electrode results in a striking feature of high over potentials for hydrogen and oxygen evolution in aqueous electrolyte which leads to the largest potential window (3.0 V) compared to the common electrodes employed such as gold, platinum ${ }^{37}$. Due to their high chemical stability, diamond electrodes can be employed in harsher environments than other carbon based electrodes.

Though boron doped diamond electrode has been studied extensively ${ }^{140}$, the electrochemical characteristics phosphorus doped diamond films or nanocrystals was not explored so far. Phosphorus/nitrogen doped individual diamond nanocrystal could serve as an excellent electrode material towards biological sensing at nanosclae with high sensitivity owing to their superior conductivity values and reduced size effects. 


\section{REFERENCES}

1. National energy flow chart by lawrence livermore national laboratory based on the work performed for U.S. department of energy DOE/EIA-0384 (2010). 2011.

2. Goldsby, J. C., Direct Electricity From Heat: A Solution to Assist Aircraft Power Demands. 2010.

3. Li, J.; Robinson, V. S.; Liu, Y.; Lu, W. J.; Fisher, T. S.; Lukehart, C. M., Synthesis and thermionic emission properties of graphitic carbon nanofibres supported on Si wafers or carbon felt. Nanotechnology 2007, 18 (32).

4. Robinson, V. S.; Show, Y.; Swain, G. M.; Reifenberger, R. G.; Fisher, T. S., Thermionic emission from surface-terminated nanocrystalline diamond. Diamond and Related Materials 2006, 15 (10), 1601-1608.

5. Redhead, P. A., The birth of electronics: Thermionic emission and vacuum. Journal of Vacuum Science \& Technology A: Vacuum, Surfaces, and Films 1998, 16 (3), 1394-1401.

6. Reimann, A. L., Thermionic Emission. John Wiley and Sons, Inc. 1934, New York, .

7. Ro, J., A review of thermionic cathodes. Vacuum 1969, 19 (8), 353-359.

8. Field, J. E., The properties of diamond. Academic $\operatorname{Pr} 1979$. 
9. Pinault, M. A.; Barjon, J.; Kociniewski, T.; Jomard, F.; Chevallier, J., The n-type doping of diamond: Present status and pending questions. Physica B-Condensed Matter 2007, 401, 51-56.

10. Suzuki, M.; Koizumi, S.; Katagiri, M.; Ono, T.; Sakuma, N.; Yoshida, H.; Sakai, T.; Uchikoga, S., Electrical characteristics of n-type diamond Schottky diodes and metal/diamond interfaces. Phys. Status Solidi A-Appl. Mat. 2006, 203 (12), 3128-3135.

11. Horiuchi, K.; Kawamura, A.; Ide, T.; Ishikura, T.; Takamura, K.; Yamashita, S., Efficient free-exciton recombination emission from diamond diode at room temperature. Jpn. J. Appl. Phys. Part 2 - Lett. 2001, 40 (3B), L275-L278.

12. Hayashi, K.; Tachibana, T.; Kawakami, N.; Yokota, Y.; Kobashi, K.; Ishihara, H.; Uchida, K.; Nippashi, K.; Matsuoka, M., Durable ultraviolet sensors using highly oriented diamond films. Diamond and Related Materials 2006, 15 (4-8), 792-796.

13. Himpsel, F. J.; Knapp, J. A.; Vanvechten, J. A.; Eastman, D. E., Quantum photoyiedl of diamond (111) -stable negative-affinity emitter Phys. Rev. B 1979, 20 (2), $624-627$

14. Mainwood, A., Theoretical modelling of dopants in diamond. J. Mater. Sci.Mater. Electron. 2006, 17 (6), 453-458.

15. Nemanich, R. J.; Bergman, L.; Turner, K. F.; Vanderweide, J.; Humphreys, T. P., Properites of interfaces of diamond Physica B 1993, 185 (1-4), 528-538.

16. Benndorf, C.; Hadenfeldt, S.; Luithardt, W.; Zhukov, A., Photoelectron spectroscopic investigations and exoelectron emission of CVD diamond surfaces modified with oxygen and potassium. Diamond and Related Materials 1996, 5 (6-8), 784-789. 
17. Kato, H.; Yamasaki, S.; Okushi, H., n-type conductivity of phosphorus-doped homoepitaxial single crystal diamond on (001) substrate. Diamond and Related Materials 2005, 14 (11-12), 2007-2010.

18. Nishimori, T.; Nakano, K.; Sakamoto, H.; Takakuwa, Y.; Kono, S., N-type highconductive epitaxial diamond film prepared by gas source molecular beam epitaxy with methane and tri-n-butylphosphine. Appl. Phys. Lett. 1997, 71 (7), 945-947.

19. Musa, G.; Mausbach, M., Sudies on thermionic cathode anodic vacuum arcs $J$. Vac. Sci. Technol. A-Vac. Surf. Films 1994, 12 (5), 2887-2895.

20. Aamodt, R. L.; Brown, L. J.; Nichols, B. D., Thermionic Emission from Molybdenum in Vapors of Cesium and Cesium Fluoride. J. Appl. Phys. 1962, 33 (6), 2080-2085.

21. Kulvarskaya, B. S.; marchenko, V. B.; stepanov, G. S., Emission properties of rare-earth metal oxides. Radio engineering and electronics 1958, III (8), 1005-1009.

22. Ahmed, H.; Broers, A. N., Lanthanum hexaboride electron emitter. J. Appl. Phys. 1972, $43(5), 2185-\&$.

23. Khairnar, R. S.; Mahajan, P. W.; Joag, D. S.; Nigavekar, A. S.; Kanitkar, P. L., Processing and characterization of LaB6-coated hairpin cathodes J. Vac. Sci. Technol. AVac. Surf. Films 1985, 3 (2), 398-402.

24. Mroczkowski, S. J., Electron-emission characteristics of sputtered lanthanum hexaboride J. Vac. Sci. Technol. A-Vac. Surf. Films 1991, 9 (3), 586-590.

25. Paramonov, D. V.; ElGenk, M. S., A review of cesium thermionic converters with developed emitter surfaces. Energy Conv. Manag. 1997, 38 (6), 533-549. 
26. Koeck, F. A. M.; Nemanich, R. J.; Balasubramaniam, Y.; Haenen, K.; Sharp, J., Enhanced thermionic energy conversion and thermionic emission from doped diamond films through methane exposure. Diamond and Related Materials 2011, 20 (8), 12291233.

27. Liu, P.; Sun, Q.; Zhu, F.; Liu, K.; Jiang, K.; Liu, L.; Li, Q.; Fan, S., Measuring the Work Function of Carbon Nanotubes with Thermionic Method. Nano Letters 2008, 8 (2), $647-651$

28. Suzuki, S.; Bower, C.; Watanabe, Y.; Zhou, O., Work functions and valence band states of pristine and Cs-intercalated single-walled carbon nanotube bundles. Appl. Phys. Lett. 2000, 76 (26), 4007-4009.

29. Suzuki, S.; Maeda, F.; Watanabe, Y.; Ogino, T., Electronic structure of singlewalled carbon nanotubes encapsulating potassium. Phys. Rev. B 2003, 67 (11).

30. Robinson, V. S.; Fisher, T. S.; Michel, J. A.; Lukehart, C. M., Work function reduction of graphitic nanofibers by potassium intercalation. Appl. Phys. Lett. 2005, 87 (6).

31. Westover, T. L.; Franklin, A. D.; Cola, B. A.; Fisher, T. S.; Reifenberger, R. G., Photo- and thermionic emission from potassium-intercalated carbon nanotube arrays. $J$. Vac. Sci. Technol. B 2010, 28 (2), 423-434.

32. Jin, F.; Liu, Y.; Day, C. M., Thermionic emission from carbon nanotubes with a thin layer of low work function barium strontium oxide surface coating. Appl. Phys. Lett. 2006, 88 (16). 
33. Vanderweide, J.; Nemanich, R. J., Infulence of interfacial hydrogen and oxygen on the schottky-barrier height of nickel on (111) and (100) diamond surfaces Phys. Rev. B 1994, $49(19), 13629-13637$.

34. Geis, M. W.; Twichell, J. C.; Macaulay, J.; Okano, K., Electron field-emission from diamond and other carbon materials after H-2, O-2 and Cs treatment Appl. Phys. Lett. 1995, 67 (9), 1328-1330.

35. Wong, K. W.; Wang, Y. M.; Lee, S. T.; Kwok, R. W. M., Negative electron affinity on polycrystalline diamond surface induced by lithium fluoride deposition. Diamond and Related Materials 1999, 8 (10), 1885-1890.

36. Wong, K. W.; Wang, Y. M.; Lee, S. T.; Kwok, R. W. M., Lowering of work function induced by deposition of ultra-thin rubidium fluoride layer on polycrystalline diamond surface. Appl. Surf. Sci. 1999, 140 (1-2), 144-149.

37. Kraft, A., Doped diamond: A compact review on a new, versatile electrode material. Int. J. Electrochem. Sci. 2007, 2 (5), 355-385.

38. Farrer, R. G., On the substitutional nitrogen donor in diamond. Solid State Communications 1969, 7 (9), 685-688.

39. Eaton, S. C.; Anderson, A. B.; Angus, J. C.; Evstefeeva, Y. E.; Pleskov, Y. V., Co-doping of diamond with boron and sulfur. Electrochem. Solid State Lett. 2002, 5 (8), G65-G68.

40. Hu, X. J.; Li, R. B.; Shen, H. S.; Dai, Y. B.; He, X. C., Electrical and structural properties of boron and phosphorus co-doped diamond films. Carbon 2004, 42 (8-9), 1501-1506. 
41. Sque, S. J.; Jones, R.; Goss, J. P.; Briddon, P. R., Shallow donors in diamond: Chalcogens, pnictogens, and their hydrogen complexes. Phys. Rev. Lett. 2004, 92 (1).

42. Goss, J. P.; Briddon, P. R., Theoretical study of Li and $\mathrm{Na}$ as $\mathrm{n}$-type dopants for diamond. Phys. Rev. B 2007, 75 (7).

43. Wang, L. G.; Zunger, A., Phosphorus and sulphur doping of diamond. Phys. Rev. $B$ 2002, $66(16)$.

44. Koizumi, S.; Kamo, M.; Sato, Y.; Ozaki, H.; Inuzuka, T., Growth and characterization of phosphorous doped $\{111\}$ homoepitaxial diamond thin films. Appl. Phys. Lett. 1997, 71 (8), 1065-1067.

45. Kuriyama, K.; Kimura, C.; Koizumi, S.; Kamo, M.; Sugino, T., Elucidation of field emission characteristics of phosphorous-doped diamond films. J. Vac. Sci. Technol. $B$ 1999, 17 (2), 723-727.

46. Chen, C. F.; Tsai, C. L.; Lin, C. L., Electronic properties of phosphorus-doped triode-type diamond field emission arrays. Mater. Chem. Phys. 2001, 72 (2), 210-213.

47. Ghodbane, S.; Omnes, F.; Bustarret, E.; Tavares, C.; Jomard, F., n-type phosphorus-doped polycrystalline diamond on silicon substrates. Diamond and Related Materials 2008, 17 (7-10), 1324-1329.

48. Uppireddi, K.; Westover, T. L.; Fisher, T. S.; Weiner, B. R.; Morell, G., Thermionic emission energy distribution from nanocrystalline diamond films for direct thermal-electrical energy conversion applications. J. Appl. Phys. 2009, 106 (4).

49. Koeck, F. A. M.; Nemanich, R. J., Sulfur doped nanocrystalline diamond films as field enhancement based thermionic emitters and their role in energy conversion. Diamond and Related Materials 2005, 14 (11-12), 2051-2054. 
50. Kataoka, M.; Zhu, C. Y.; Koeck, F. A. M.; Nemanich, R. J., Thermionic electron emission from nitrogen-doped homoepitaxial diamond. Diamond and Related Materials 2010, $19(2-3), 110-113$.

51. Suzuki, M.; Ono, T.; Sakuma, N.; Sakai, T., Low-temperature thermionic emission from nitrogen-doped nanocrystalline diamond films on n-type $\mathrm{Si}$ grown by MPCVD. Diamond and Related Materials 2009, 18 (10), 1274-1277.

52. Koeck, F. A. M.; Nemanich, R. J., Low temperature onset for thermionic emitters based on nitrogen incorporated UNCD films. Diamond and Related Materials 2009, 18 $(2-3), 232-234$.

53. Koeck, F. A. M.; Nemanich, R. J.; Lazea, A.; Haenen, K., Thermionic electron emission from low work-function phosphorus doped diamond films. Diamond and Related Materials 2009, 18 (5-8), 789-791.

54. Kawano, H.; Matsui, S.; Kobayashi, H.; Zhu, Y. F., Effective work functions for thermal positive-ionic and electronic emissions from tantalum heated in a high vacuum. Vacuum 1997, 48 (7-9), 629-631.

55. Kawano, H., Effective work functions for ionic and electronic emissions from mono- and polycrystalline surfaces. Prog. Surf. Sci. 2008, 83 (1-2), 1-165.

56. Xu, N. S.; Chen, Y.; Deng, S. Z.; Chen, J.; Ma, X. C.; Wang, E. G., Vacuum gap dependence of field electron emission properties of large area multi-walled carbon nanotube films. Journal of Physics D-Applied Physics 2001, 34 (11), 1597-1601.

57. Nilsson, L.; Groening, O.; Emmenegger, C.; Kuettel, O.; Schaller, E.; Schlapbach, L.; Kind, H.; Bonard, J. M.; Kern, K., Scanning field emission from patterned carbon nanotube films. Appl. Phys. Lett. 2000, 76 (15), 2071-2073. 
58. Chen, Z.; Wei, Y.; Luo, C. X.; Jiang, K. L.; Zhang, L.; Li, Q. Q.; Fan, S. S.; Gao, J. C., Laser direct writing carbon nanotube arrays on transparent substrates. Appl. Phys. Lett. 2007, $90(13)$.

59. Fujii, S.; Honda, S.; Machida, H.; Kawai, H.; Ishida, K.; Katayama, M.; Furuta, H.; Hirao, T.; Oura, K., Efficient field emission from an individual aligned carbon nanotube bundle enhanced by edge effect. Appl. Phys. Lett. 2007, 90 (15).

60. Milne, W. I.; Teo, K. B. K.; Amaratunga, G. A. J.; Legagneux, P.; Gangloff, L.; Schnell, J. P.; Semet, V.; Binh, V. T.; Groening, O., Carbon nanotubes as field emission sources. Journal of Materials Chemistry 2004, 14 (6), 933-943.

61. Bonard, J. M.; Kind, H.; Stockli, T.; Nilsson, L. A., Field emission from carbon nanotubes: the first five years. Solid-State Electron. 2001, 45 (6), 893-914.

62. Bonard, J. M.; Salvetat, J. P.; Stockli, T.; de Heer, W. A.; Forro, L.; Chatelain, A., Field emission from single-wall carbon nanotube films. Appl. Phys. Lett. 1998, 73 (7), $918-920$.

63. Sveningsson, M.; Morjan, R. E.; Nerushev, O. A.; Sato, Y.; Backstrom, J.; Campbell, E. E. B.; Rohmund, F., Raman spectroscopy and field-emission properties of CVD-grown carbon-nanotube films. Applied Physics a-Materials Science \& Processing 2001, $73(4), 409-418$

64. Li, J. J.; Wang, Q.; Gu, C. Z., Growth and field emission properties of tubular carbon cones. Ultramicroscopy 2007, 107 (9), 861-864.

65. Iijima, S., Helical microtubules of graphitic carbon. Nature 1991, 354 (6348), 5658. 
66. Krishnan, A.; Dujardin, E.; Treacy, M. M. J.; Hugdahl, J.; Lynum, S.; Ebbesen, T. W., Graphitic cones and the nucleation of curved carbon surfaces. Nature 1997, 388 (6641), 451-454.

67. Gogotsi, Y.; Dimovski, S.; Libera, J. A., Conical crystals of graphite. Carbon 2002, 40 (12), 2263-2267.

68. Mani, R. C.; Li, X.; Sunkara, M. K.; Rajan, K., Carbon nanopipettes. Nano Letters 2003, 3 (5), 671-673.

69. Zhang, G.; Jiang, X.; Wang, E., Tubular Graphite Cones. Science 2003, 300 $(5618), 472-74$.

70. Merkulov, V. I.; Guillorn, M. A.; Lowndes, D. H.; Simpson, M. L.; Voelkl, E., Shaping carbon nanostructures by controlling the synthesis process. Appl Phys Lett 2001, $79(8), 1178-80$.

71. Xia, W.; Su, D.; Schlögl, R.; Birkner, A.; Muhler, M., Conical Carbon Filaments with Axial Cylindrical Channels and Open Tips. Adv Mater 2005, 17 (13), 1677-79.

72. Muradov, N.; Schwitter, A., Formation of Conical Carbon Structures on VaporGrown Carbon Filaments. Nano Lett 2002, 2 (6), 673-676.

73. Zhang, W.; Xi, Z. H.; Zhang, G. M.; Wang, S.; Wang, M. S.; Wang, J. Y.; Xue, Z. Q., Carbon nanotube as the core of conical carbon fiber: fabrication, characterization and field emission property. Appl Phys A-Mater Sci Process 2007, 86 (2), 171-175.

74. lijima, S.; Ichihashi, T.; Ando, Y., Pentagons, heptagons and negative curvature in graphite microtubule growth. Nature 1992, 356 (6372), 776-8. 
75. Lowe, R. D.; Mani, R. C.; Baldwin, R. P.; Sunkara, M. K., Nanoelectrode ensembles using carbon nanopipettes. Electrochem. Solid State Lett. 2006, 9 (6), H43H47.

76. Dumpala, S.; Safir, A.; Mudd, D.; Cohn, R. W.; Sunkara, M. K.; Sumanasekera, G. U., Controlled synthesis and enhanced field emission characteristics of conical carbon nanotubular arrays. Diam Relat Mater 2009, 18 (10), 1262-1266.

77. Henry, S.; McAllister, D. V.; Allen, M. G.; Prausnitz, M. R., Microfabricated microneedles: A novel approach to transdermal drug delivery. Journal of Pharmaceutical Sciences 1998, $87(8), 922-925$.

78. Shang, N. G.; Jiang, X., Large-sized tubular graphite cones with nanotube tips. Appl Phys Lett 2005, 87 (16), 163102-3.

79. Shang, N. G.; Papakonstantinou, P.; McLaughlin, J.; Chen, W. C.; Chen, L. C.; Chu, M.; Stamboulis, A., Fe catalytic growth, microstructure, and low-threshold field emission properties of open ended tubular graphite cones. J Appl Phys 2008, 103 (12), 124308-4.

80. Chung, P. H.; Perevedentseva, E.; Tu, J. S.; Chang, C. C.; Cheng, C. L., Spectroscopic study of bio-functionalized nanodiamonds. Diamond and Related Materials 2006, 15 (4-8), 622-625.

81. Subramanian, K.; Kang, W. P.; Davidson, J. L.; Hofmeister, W. H.; Choi, B. K.; Howell, M., Nanodiamond planar lateral field emission diode. Diamond and Related Materials 2005, 14 (11-12), 2099-2104. 
82. Arenal, R.; Bruno, P.; Miller, D. J.; Bleuel, M.; Lal, J.; Gruen, D. M., Diamond nanowires and the insulator-metal transition in ultrananocrystalline diamond films. Phys. Rev. $B$ 2007, 75 (19).

83. Vlasov, I. L.; Lebedev, O. I.; Ralchenko, V. G.; Goovaerts, E.; Bertoni, G.; Van Tendeloo, G.; Konov, V. I., Hybrid diamond-graphite nanowires produced by microwave plasma chemical vapor deposition. Advanced Materials 2007, 19 (22), 4058-+.

84. Lambrecht, W. R. L.; Lee, C. H.; Segall, B.; Angus, J. C.; Li, Z. D.; Sunkara, M., Diamond nucleation by hydrogenation of the edges of graphitic precursors Nature 1993, $364(6438), 607-610$.

85. Mehandru, S. P.; Anderson, A. B.; Angus, J. C., Hydrogenation of the (10(1)baro) graphite edge-structural considerations from band calculations Journal of Physical Chemistry 1992, $96(26), 10978-10982$.

86. Li, Z. D.; Wang, L.; Suzuki, T.; Argoitia, A.; Pirouz, P.; Angus, J. C., Orientation relationship between chemical vapor-deposited diamond and graphite substrates. J. Appl. Phys. 1993, 73 (2), 711-715.

87. Sun, L. T.; Gong, J. L.; Zhu, Z. Y.; Zhu, D. Z.; He, S. X.; Wang, Z. X.; Chen, Y.; Hu, G., Nanocrystalline diamond from carbon nanotubes. Appl. Phys. Lett. 2004, 84 (15), 2901-2903.

88. Sun, L. T.; Gong, J. L.; Zhu, D. Z.; Zhu, Z. Y.; He, S. X., Diamond nanorods from carbon nanotubes. Advanced Materials 2004, 16 (20), 1849-+.

89. Lifshitz, Y.; Kohler, T.; Frauenheim, T.; Guzmann, I.; Hoffman, A.; Zhang, R. Q.; Zhou, X. T.; Lee, S. T., The mechanism of diamond nucleation from energetic species. Science 2002, 297 (5586), 1531-1533. 
90. Lifshitz, Y.; Meng, X. M.; Lee, S. T.; Akhveldiany, R.; Hoffman, A., Visualization of diamond nucleation and growth from energetic species. Phys. Rev. Lett. 2004, $93(5)$.

91. Terranova, M. L.; Orlanducci, S.; Fiori, A.; Tamburri, E.; Sessa, V.; Rossi, M.; Barnard, A. S., Controlled evolution of carbon nanotubes coated by nanodiamond: the realization of a new class of hybrid nanomaterials. Chemistry of Materials 2005, 17 (12), $3214-3220$.

92. Barnard, A. S.; Terranova, M. L.; Rossi, M., Density functional study of Hinduced defects as nucleation sites in hybrid carbon nanomaterials. Chemistry of Materials 2005, $17(3), 527-535$.

93. Cowley, J. M.; Mani, R. C.; Sunkara, M. K.; O'Keeffe, M.; Bonneau, C., Structures of carbon nanocrystals. Chemistry of Materials 2004, 16 (24), 4905-4911.

94. Conrads, H.; Schmidt, M., Plasma generation and plasma sources. Plasma Sources Sci. Technol. 2000, 9 (4), 441-454.

95. Kociniewski, T.; Barjon, J.; Pinault, M. A.; Jomard, F.; Lusson, A.; Ballutaud, D.; Gorochov, O.; Laroche, J. M.; Rzepka, E.; Chevallier, J.; Saguy, C., n-type CVD diamond doped with phosphorus using the MOCVD technology for dopant incorporation. Phys. Status Solidi A-Appl. Mat. 2006, 203 (12), 3136-3141.

96. Chernomordik, B.; Dumpala, S.; Chen, Z. Q.; Sunkara, M. K., Nanodiamond Tipped and Coated Conical Carbon Tubular Structures. Chem Vap Depos 2008, 14 (7-8), 256-262. 
97. Bera, D.; Perrault, J. P.; Heinrich, H.; Seal, S., Defect studies on As-synthesized and purified carbon nanostructures produced by arc-discharge in solution process. $J$ Nanosci Nanotechnol 2006, 6 (4), 1084-1091.

98. Li, H.; Zhao, N.; Wang, L.; Shi, C.; Du, X.; Li, J., Synthesis of carbon nanohorns by the simple catalytic method. J Alloy Compd 2009, 473 (1-2), 288-292.

99. Li, X.; Lei, Z.; Ren, R.; Liu, J.; Zuo, X.; Dong, Z.; Wang, H.; Wang, J., Characterization of carbon nanohorn encapsulated Fe particles. Carbon 2003, 41 (15), 3068-3072.

100. Liu, J.; Lin, W.; Chen, X.; Zhang, S.; Li, F.; Qian, Y., Fabrication of hollow carbon cones. Carbon 2004, 42 (3), 669-671.

101. Chhowalla, M.; Teo, K. B. K.; Ducati, C.; Rupesinghe, N. L.; Amaratunga, G. A. J.; Ferrari, A. C.; Roy, D.; Robertson, J.; Milne, W. I., Growth process conditions of vertically aligned carbon nanotubes using plasma enhanced chemical vapor deposition. $J$ Appl Phys 2001, $90(10), 5308-5317$.

102. Meyyappan, M.; Delzeit, L.; Cassell, A.; Hash, D., Carbon nanotube growth by PECVD: a review. Plasma Sources Sci. Technol. 2003, 12 (2), 205-216.

103. Hash, D. B.; Meyyappan, M., Model based comparison of thermal and plasma chemical vapor deposition of carbon nanotubes. J. Appl. Phys. 2003, 93 (1), 750-752.

104. Weisenberger, M.; Martin-Gullon, I.; Vera-Agullo, J.; Varela-Rizo, H.; Merino, C.; Andrews, R.; Qian, D. L.; Rantell, T., The effect of graphitization temperature on the structure of helical-ribbon carbon nanofibers. Carbon 2009, 47 (9), 2211-2218.

105. Gogotsi, Y.; Libera, J. A.; Kalashnikov, N.; Yoshimura, M., Graphite Polyhedral Crystals. Science 2000, 290 (5490), 317-320. 
106. Bacon, R., Growth, Structure, and Properties of Graphite Whiskers. J Appl Phys 1960, $31(2), 283-290$.

107. Park, J. H.; Yoon, Y. K.; Choi, S. O.; Prausnitz, M. R.; Allen, M. G., Tapered conical polymer microneedles fabricated using an integrated lens technique for transdermal drug delivery. Ieee Transactions on Biomedical Engineering 2007, 54 (5), 903-913.

108. Galvan, D. H.; Amarillas, A. P.; Elizondo, N.; Mejia, S.; Perez-Tijerina, E.; JoseYacaman, M., Diffraction Patterns Observed in Two-layered Graphene and Their Theoretical Explanation. Fuller. Nanotub. Carbon Nanostruct. 2009, 17 (3), 258-272.

109. Ong, T. S.; Yang, H., Effect of atmosphere on the mechanical milling of natural graphite. Carbon 2000, 38 (15), 2077-2085.

110. Park, H. J.; Meyer, J.; Roth, S.; Skákalová, V., Growth and properties of fewlayer graphene prepared by chemical vapor deposition. Carbon 2010, 48 (4), 1088-1094.

111. Choudhary, S. K.; Gupta, A. K., Spatially varying super-lattice structures and linear fringes on graphite surface. J. Phys.-Condes. Matter 2008, 20 (22).

112. Choudhary, S. K.; Gupta, A. K., Some unusual electronic patterns on graphite surface. Pramana-J. Phys. 2008, 70 (2), 339-350.

113. Li, J. J.; Gu, C. Z.; Wang, Q.; Xu, P.; Wang, Z. L.; Xu, Z.; Bai, X. D., Field emission from high aspect ratio tubular carbon cones grown on gold wire. Appl. Phys. Lett. 2005, 87 (14).

114. Maiti, A.; Andzelm, J.; Tanpipat, N.; von Allmen, P., Effect of adsorbates on field emission from carbon nanotubes. Phys. Rev. Lett. 2001, 87 (15). 
115. Dean, K. A.; Burgin, T. P.; Chalamala, B. R., Evaporation of carbon nanotubes during electron field emission. Appl. Phys. Lett. 2001, 79 (12), 1873-1875.

116. Gat, R.; Angus, J. C., Hydrogenation-atom recombination on tungsten and diamond in hot-filament assisted deposition of diamond J. Appl. Phys. 1993, 74 (10), 5981-5989.

117. Wolden, C.; Mitra, S.; Gleason, K. K., Radiative heat-transfer in hot-filament chemical vapor-deposition diamond reactors J. Appl. Phys. 1992, 72 (8), 3750-3758.

118. Che, J. W.; Cagin, T.; Goddard, W. A., Thermal conductivity of carbon nanotubes. Nanotechnology 2000, 11 (2), 65-69.

119. Ruffieux, P.; Groning, O.; Bielmann, M.; Mauron, P.; Schlapbach, L.; Groning, P., Hydrogen adsorption on sp(2)-bonded carbon: Influence of the local curvature. Phys. Rev. B 2002, 66 (24).

120. Ugarte, D., Curling and closure of graphitc networks under electron-beam irradition Nature 1992, 359 (6397), 707-709.

121. Sano, N.; Wang, H.; Alexandrou, I.; Chhowalla, M.; Teo, K. B. K.; Amaratunga, G. A. J.; limura, K., Properties of carbon onions produced by an arc discharge in water. $J$. Appl. Phys. 2002, 92 (5), 2783-2788.

122. Orlanducci, S.; Fiori, A.; Tamburri, E.; Sessa, V.; Terranova, M. L.; Rossi, M., Nanocrystalline non-planar carbons: Growth of carbon nanotubes and curled nanostructures. Crystal Research and Technology 2005, 40 (10-11), 928-931.

123. Robertson, D. H.; Brenner, D. W.; White, C. T., On the way to fullerenesmolecular-dynamics study of the curling and closure of graphitc ribborns Journal of Physical Chemistry 1992, 96 (15), 6133-6135. 
124. Wei, B.; Zhang, J.; Liang, J.; Wu, D., The mechanism of phase transformation from carbon nanotube to diamond. Carbon 1998, 36 (7-8), 997-1001.

125. Banhart, F.; Ajayan, P. M., Carbon onions as nanoscopic pressure cells for diamond formation. Nature 1996, 382 (6590), 433-435.

126. Huang, J. Y., In situ observation of quasimelting of diamond and reversible graphite-diamond phase transformations. Nano Letters 2007, 7 (8), 2335-2340.

127. Hamon, A. L.; Verbeeck, J.; Schryvers, D.; Benedikt, J.; Van der Sanden, R., ELNES study of carbon K-edge spectra of plasma deposited carbon films. Journal of Materials Chemistry 2004, 14 (13), 2030-2035.

128. Modinos, A., Field, thermionic and secondary electron emission spectroscopy. Plenum Press 1984, New York.

129. Koeck, F. A. M.; Nemanich, R. J., Emission characterization from nitrogen-doped diamond with respect to energy conversion. Diamond and Related Materials 2006, 15 (23), 217-220.

130. Gubbala, S.; Russell, H. B.; Shah, H.; Deb, B.; Jasinski, J.; Rypkema, H.; Sunkara, M. K., Surface properties of $\operatorname{SnO}(2)$ nanowires for enhanced performance with dye-sensitized solar cells. Energy Environ. Sci. 2009, 2 (12), 1302-1309.

131. Rentenberger, S.; Vollmer, A.; Zojer, E.; Schennach, R.; Koch, N., UV/ozone treated $\mathrm{Au}$ for air-stable, low hole injection barrier electrodes in organic electronics. $J$. Appl. Phys. 2006, $100(5)$.

132. Diederich, L.; Kuttel, O.; Aebi, P.; Schlapbach, L., Electron affinity and work function of differently oriented and doped diamond surfaces determined by photoelectron spectroscopy. Surf. Sci. 1998, 418 (1), 219-239. 
133. Diederich, L.; Kuttel, O. M.; Ruffieux, P.; Pillo, T.; Aebi, P.; Schlapbach, L., Photoelectron emission from nitrogen- and boron-doped diamond (100) surfaces. Surf. Sci. 1998, $417(1), 41-52$.

134. Cui, J. B.; Ristein, J.; Ley, L., Electron affinity of the bare and hydrogen covered single crystal diamond (111) surface. Phys. Rev. Lett. 1998, 81 (2), 429-432.

135. Maier, F.; Ristein, J.; Ley, L., Electron affinity of plasma-hydrogenated and chemically oxidized diamond (100) surfaces. Phys. Rev. B 2001, 64 (16), art. no.-165411. 136. Takeuchi, D.; Kato, H.; Ri, G. S.; Yamada, T.; Vinod, P. R.; Hwang, D.; Nebel, C. E.; Okushi, H.; Yamasaki, S., Direct observation of negative electron affinity in hydrogen-terminated diamond surfaces. Appl. Phys. Lett. 2005, 86 (15).

137. Abbott, P.; Sosa, E. D.; Golden, D. E., Effect of average grain size on the work function of diamond films. Appl. Phys. Lett. 2001, 79 (17), 2835-2837.

138. Kono, S.; Mimochi, K.; Takyo, G.; Goto, T.; Abukawa, T.; Aoyama, T., Energy band diagram of a H-terminated P-doped n-type diamond (111) surface. New Diam. Front. Carbon Technol. 2007, 17 (5), 231-242.

139. Vanderweide, J.; Nemanich, R. J., Argon and hydrogen plasma interaction on diamond (111) surfaces-electron states and structure Appl. Phys. Lett. 1993, 62 (16), 18781880.

140. Chailapakul, O.; Siangproh, W.; Tryk, D. A., Boron-doped diamond-based sensors: A review. Sens. Lett. 2006, 4 (2), 99-119. 


\section{CURRICULAM VITAE}

\section{Santoshrupa Dumpala}

\section{Education}

$\mathrm{PhD}$, Chemical Engineering

2005-2011

University of Louisville, Louisville, KY

GPA: $3.85 / 4$

Bachelor of Technology, Chemical Engineering

2001-2005

Jawaharlal Nehru Technological University, India

Marks: $83.4 \%$

\section{Achievements/Awards}

- Third prize winner at poster presentation, Renewable energy \& energy efficiency work shop, KY, 2011

- Recepient of Pullin Fellowship, University of Louisville, 2008-2010.

- Second prize at poster presentation, Nanoscale science engineering session, AIChE 2007

- Winner of poster presentation, Engineering-Exposition, University of Louisville, 2006-2010.

- Secretary of Chemical Engineering Graduate Students Association, University of Louisville 2007-2008.

- Secretary for India Student Association (ISA), University of Louisville, 20062007.

- Trainee of Six Sigma Program

\section{Experience}

Teaching/Research Assistant

University of Louisville

$2006-2011$

- Performed research using plasma enhanced and other vapor deposition of novel carbon structures and thorough characterization of carbon nanostructures using various surface sciences, structural and thermionic emission techniques.

- Assisted in teaching graduate courses such as Transport Phenomena and undergraduate courses such as Reaction Kinetics and Computer Applications in Chemical Engineering. 
- Conducted labs for 400 students on "Chemical Solar Cells" as part of freshman course.

- Mentored 2 high school students for national level science fair.

Undergraduate Internship Ramagundam, India Jan 2005-May 2005

- Air and water pollution monitoring at National Thermal Power Corporation

\section{Patents}

"Conical carbon nanopipettes: Method of making and applications", M. K. Sunkara, $\underline{\text {. }}$ Dumpala, R. C. Mani, R. D. Lowe, R. P. Baldwin and G. U. Sumanasekera, US utility patent application \# 12176632, Jun, 2008 ( cont'd patent from 2004).

\section{Publications}

1. S. Dumpala, A. Sherehiy, J. B. Jasinski, G. U. Sumanasekera and M. K. Sunkara; "Thermionic emission from phosphorus doped diamond tipped carbon hybrid architectures", Manuscript in preparation (2011)

2. S. Dumpala, A. Safir, D. Mudd, R. W. Cohn, M. K. Sunkara, G. U. Sumanasekera; "Thermionic emission properties and work function determination of an array of conical carbon nanotubes grown by CVD" manuscript in preparation (2011).

3. J.B. Jasinski, S. Dumpala, G. Sumanasekera, M. K. Sunkara and P.J. Ouseph; "O bservation and interpretation of adjacent moiré patterns of different shapes", Applied Physics Letters, 99, DOI: 10.1063/1.3624703, (2011).

4. S. Dumpala, J.Jasinski, G.U. Sumanasekera, M.K. Sunkara, "Large area synthesis of conical carbon nanotube arrays on foil substrates", Carbon, 49, 2725 (2011).

5. S. Dumpala, A. Safir, D. Mudd, R.W. Cohn, M.K. Sunkara, G.U. Sumanasekera, "Controlled synthesis and enhanced field emission characteristics of conical carbon nanotubular arrays", Diamond and Related Materials, 18, 10 (2009).

6. B. Chernomordik, S. Dumpala, Z.Q. Chen, M.K. Sunkara, "Nanodiamond tipped and Coated Conical Carbon Tubular Structures", Chemical Vapor Deposition, 14, 7 (2008). 
7. Book Chapter S. Dumpala, G. Bhimarasetti, S. Gubbala, P. Meduri, S. Kona and M.K. Sunkara; "Carbon microtubes and conical carbon nanotubes", Smart Materials edited by Mel Schwartz, Taylor \& Francis Group, CRC Press, (2009).

\section{Presentations}

1. Thermionic emission and Field emission properties of Carbon nanopipettes, Poster presentation, $15^{\text {th }}$ Annual KY EPSCoR conference, Lexington, KY, May 2010.

2. Conical Carbon Nanotubes Arrays: Large area synthesis, Electrochemical and Field emission characteristics, Oral presentation, AIChE Annual meeting, Nashville, TN, Nov. 2009.

3. Conical Carbon Nanotubes Arrays: Large area synthesis, Electrochemical and Field emission characteristics, Oral presentation, AIChE Annual meeting, Philadelphia, PA, Nov. 2008.

4. Field Emission Properties of Carbon Nano Pipettes, Poster presentation, DOE EPSCoR meeting, Oak Rigde, TN, July 2008.

5. Nucleation and Electrochemical Studies of Diamond on Conical Carbon Nanotubular Arrays, Poster presentation, AICHE Annual meeting, Salt Lake City, UT, Nov. 2007.

6. Diamond Nanotip Array Electrodes, Oral presentation, Electrochemical Society Annual Fall meeting, Washington DC, Oct. 2007.

7. Nano Diamond Tip Array Electrode, Oral presentation, Material Research Society Annual meeting, Boston, MA, Nov. 2006.

\section{Membership/Affiliations: AIChE}

\section{Technical Expertise}

- Reactors: Microwave and electron cyclotron resonance plasma enhanced chemical vapor deposition (CVD), hot filament CVD reactors, expertise in handling the metal organic CVD precursors and vapor pressure measurements.

- Material characterization techniques: Scanning electron microscope (SEM), X-ray photoelectron spectroscopy (XPS), Ultraviolet photoelectron spectroscopy (UPS), Energy dispersive spectroscopy (EDS), X-Ray diffraction (XRD), Raman spectroscopy, Photoluminescence, Transmission electron microscope (TEM). 
- Other analysis instruments: Electrochemical impedance spectroscopy, Residual gas analysis, Optical emission spectroscopy (OES), Atomic layer deposition (ALD), UV-Vis, FTIR.

- Thermo electric power, field and thermionic emission measurements.

- Application software: CHEMKIN, POLYMATH, Peak fit, Digital Micrograph, Origin 